\title{
Targeted Lipid-Coated Nanoparticles: Delivery of Tumor Necrosis Factor- Functionalized Particles to Tumor Cells
}

\author{
Von der Fakultät Energie-, Verfahrens- und Biotechnik der \\ Universität Stuttgart zur Erlangung der Würde eines Doktors der \\ Naturwissenschaften (Dr. rer. nat.) genehmigte Abhandlung
}

\author{
Vorgelegt von \\ Sylvia Messerschmidt \\ aus Neustadt an der Weinstraße
}

Hauptberichter: Prof. Dr. Roland Kontermann

Mitberichter: $\quad$ Prof. Dr. Peter Scheurich

Tag der mündlichen Prüfung: 06. Juli 2009

Institut für Zellbiologie und Immunologie

Universität Stuttgart

2009 
I hereby assure that I performed the present study independently without further help or other materials than stated.

Sylvia Messerschmidt

Stuttgart, $14^{\text {th }}$ of April 2009 


\section{Table of contents}

Table of contents

Abbreviations

Summary

Zusammenfassung

1 Introduction

1.1 The Tumor Necrosis Factor (TNF) and the TNF Receptor Superfamily 11

1.2 The TNF Receptor Signal-Transduction Pathways 12

1.3 Cellular and Antitumoral Effects of TNF 15

1.4 Single-Chain TNF 17

1.5 Liposomes and Polymeric Nanoparticles 17

1.6 Immunoliposomes 19

1.7 Aim of the Study 22

2 Materials and Methods 24

2.1 Materials 24

2.1.1 Instruments and Special Implements __ 24

2.1.2 Chemicals and Lipids __ 26

2.1.3 Cell lines__ 27

2.1.4 Bacterial Strain E. coli TG1 27

2.1.5 Media and Supplements___ 27

2.1.6 Solutions___ 28

2.1.7 Antibodies, Enzymes, Kits, Markers etc. _ 28

2.1.8 Primers_ 29

2.1.9 Vectors 30

2.2 Methods 32

2.2.1 Cloning of scFv' variants 32

2.2.1.1 Cloning strategies for the scFv' constructs _ 32

2.2.1.2 Polymerase Chain Reaction (PCR)___ 32

2.2.1.3 Restriction Digestion ___ 33

2.2.1.4 Agarose Gel Electrophoresis and DNA Gel Extraction ___ 33

2.2.1.5 Ligation _ 33

2.2.1.6 Transformation of E. coli TG1 _ 34 
2.2.1.7 Screening of Clones _ 34

2.2.1.8 Plasmid-DNA Isolation (Midi) __ 34

2.2.1.9 Sequence Analysis ___ 34

2.2.1.10 Photometric Measurement of DNA concentration___ 34

2.2.2 Expression and Purification of scFv' variants __ 35

2.2.2.1 Periplasmic protein expression in E. coli__ 35

2.2.2.2 Purification by Immobilized Metal Affinity Chromatography (IMAC) _ 35

2.2.3 Protein Characterization___ 36

2.2.3.1 Determination of Protein Concentration _ 36

2.2.3.2 SDS-PAGE and Western Blot Analysis__ 36

2.2.3.3 Western Blot Analysis ___ 36

2.2.3.4 Size Exclusion Chromatography by High Performance Liquid Chromatography _ 37

2.2.3.5 Determination of Protein Melting Points _ـ 37

2.2.4 Liposome Technology __ 37

2.2.4.1 Liposome Preparation __ 37

2.2.4.2 Coupling scFv' to Micelles__ 38

2.2.4.3 Analysis of Coupling Efficiency ___ 38

2.2.4.4 Generation of Immunoliposomes __ 38

2.2.4.5 Determination of Liposome Size and $\zeta$-potential ___ 38

2.2.5 Lipid coating of Nanoparticles _ 39

2.2.5.1 Lipid Coating by Extrusion __ 39

2.2.5.2 Lipid Coating by Sonification___ 39

2.2.6 Generation of Targeted Lipid-Coated Nanoparticles _ 39

2.2.7 Binding Studies_ 40

2.2.7.1 Cell culture — 40

2.2.7.2 Flow Cytometry __ 40

2.2.7.3 In Vitro Plasma Stability __ 40

2.2.7.4 Internalization Studies by Microscopy___ 41

2.2.8 Pharmacokinetic___ 42

2.2.9 Coupling of Cys-scTNF to Nanoparticles__ 42

2.2.10 Cytotoxicity Assays___ 43

3 Results

\subsection{Novel Single-Chain Fv' Formats for the Generation of Immunoliposomes by} Site-Directed Coupling

3.1.1 Generation and Characterization of scFv' Variants __ 44

3.1.2 Coupling of scFv' Fragments to Mal-PEG 2000 -DSPE Micelles __ 48

3.1.3 Generation of ScFv Immunoliposomes by the Postinsertion Method _ 49

3.1.4 ScFv' Formats Combining a Cysteine Residue and Hexahistidyl-Tag in the Linker _ 52 
3.2 Targeted Lipid-Coated Nanoparticles: Delivery of Tumor Necrosis FactorFunctionalized Particles to Tumor Cells 56

3.2.1 Establishment of Methods for Lipid Coating of Nanoparticles __ 56

3.2.1.1 Lipid-Coating by Extrusion __ 56

3.2.1.2 Lipid-Coating by Sonification__ 57

3.2.2 Targeted Lipid-Coated Nanoparticles (TLP)___ 59

3.2.2.1 Binding Studies of Targeted Lipid-Coated Particles ___ 59

3.2.2.2 Internalization Studies by Fluorescence Microscopy ___ 64

3.2.2.3 Pharmacokinetic Properties _ 65

3.2.3 Generation of scTNF-Nanoparticles __ 66

3.2.3.1 Cys-scTNF _ 66

3.2.3.2 Coupling of Cys-scTNF to Amino-Functionalized Nanoparticles___ 67

3.2.4 Cys-scTNF-functionalized TLP__ 69

3.2.4.1 Binding Studies of anti-FAP scTNF-TLP__ 69

3.2.4.2 Internalization Studies of anti-FAP scTNF-TLP by Confocal Microscopy __ 70

3.2.4.3 Cytotoxicity Assays on Kym-1 cells___ 71

3.2.4.4 Cytotoxicity Assays on HT1080 cells 72

4 Discussion 73

4.1 Novel scFv' Formats for the Generation of Targeted Carrier Systems__ 73

4.1.1 $\mathrm{HC}$ and LC Variants _ 74

4.1.2 LCH Variants _ 76

4.2 Targeted Composite Nanoparticles _ 78

4.2.1 Generation of Lipid-Coated Particles _ 78

4.2.2 Generation of Targeted Lipid-Coated Particles _ 80

4.2.3 Cell Death Mediated by scTNF-Functionalized Nanoparticles ___ 81

4.2.4 Generation of Anti-FAP scTNF-Targeted Lipid-Coated Particles ___ 83

Bibliography _ 88

Appendix $\quad 99$

Acknowledgements 106

Curriculum Vitae 1107 


\section{Abbreviations}

${ }^{\circ} \mathrm{C} \quad$ degree Celsius

$\mu \mathrm{g}$ microgram

$\mu \mathrm{l} \quad$ microliter

$\mu \mathrm{m} \quad$ micrometer $\left(10^{-6} \mathrm{~m}\right)$

$\mu \mathrm{M} \quad$ micromolar $\left(10^{-6} \mathrm{M}\right)$

a anti

aa amino acid

$\mathrm{Ab} / \mathrm{mAb}$ (monoclonal) antibody

AFM atomic force microscopy

AIDS acquired immune deficiency

syndrome

amp ampicillin

APS Ammonium persulfate

$\mathrm{Bp} \quad$ base pair

BSA bovine serum albumin

CD cluster of differentiation

CEA carcinoembryonic antigen

cFLIP cellular FLICE inhibitory

protein

EGF Epidermal Growth Factor

EPC egg phosphatidyl choline

f.c. final concentration

Fab fragment antigen binding

FADD fas-associating protein with a death domain

Fas/ $\quad$ FS-7 cell-associated surface

FasL antigen/ ligand

FAP fibroblast activation protein

Fc fragment crystallizable

FCS fetal calf serum

FDA Food and Drug Administration

FITC fluorescein isothiocyanate

g gram

h hour

hu human

HEPES 4-(2-hydroxyethyl)-1piperazineethanesulfonic acid

HPLC high performance liquid

Chol cholesterol

CRD cysteine rich domain

HRP horse radish peroxidase

cys- cysteine single-chain TNF

ScTNF

Ig Immunoglobulin

IgG Immunoglobulin $G$

$\mathrm{Da} / \mathrm{kDa} \quad$ (kilo) Dalton

DMSO dimethyl sulfoxide

IKB inhibitor of $\mathrm{KB}$

DNA deoxyribonucleic acid

IMAC Immobilized metal affinity

dNTP deoxyribonucleoside

JNK

chromatography

triphosphate

I

c-Jun N-terminal kinase

DSPE distearoyl-
phosphoethanolamine

LP

liter

DTT dithiothreitol

Lum Lumogen $F$ red

ECL enhanced chemiluminescence

M molar

EDTA ethylenediaminetetraacetate

$\mathrm{mA}$ milliampere

MAPK mitogen activated protein kinase 


\begin{tabular}{|c|c|c|c|}
\hline MCS & multiple cloning site & $s$ & second \\
\hline $\mathrm{mg}$ & milligram & scFv & single-chain fragment variable \\
\hline $\min$ & minute & scTNF & single chain TNF \\
\hline $\mathrm{ml}$ & milliliter & scTNF-P & scTNF-functionalized particle \\
\hline $\mathrm{mM}$ & millimolar $\left(10^{-3} \mathrm{M}\right)$ & SDS & sodium dodecyl sulfate \\
\hline MMP & matrix metallo-proteinase & sTNF & soluble TNF \\
\hline mo & mouse & TACE & TNFa converting enzyme \\
\hline mTNF & membrane bound TNF & TAE & tris-actetate-EDTA \\
\hline MW & molecular mass & TEMED & $\mathrm{N}, \mathrm{N}, \mathrm{N}^{\prime}, \mathrm{N}^{\prime}$-tetramethylethyl- \\
\hline MWCO & molecular weight cut-off & & diamine \\
\hline NF-kB & nuclear factor-kappa B & TNF & tumor necrosis factor \\
\hline $\mathrm{nm}$ & nanometer $\left(10^{-9} \mathrm{~m}\right)$ & TNFR & TNF receptor \\
\hline \multirow[t]{2}{*}{ NSL } & aminomonomer AEMH styrene & TRADD & TNFR associated death domain \\
\hline & Lutensol AT50 & TRAF & TNFR associated factor \\
\hline $\mathrm{nM}$ & nanomolar $\left(10^{-9} \mathrm{M}\right)$ & TRAIL & TNF-related apoptosis-inducing \\
\hline OD & optical density & & ligand \\
\hline$P$ & particle & Tris & tris-(hydroxymethyl)-amino- \\
\hline PEG & poly-(ethylene glycol) & & methane \\
\hline mPEG & methoxy-poly-(ethylene glycol) & $\mathrm{V}_{\mathrm{H}}, \mathrm{V}_{\mathrm{L}}$ & variable domain of heavy and \\
\hline \multirow[t]{2}{*}{ MalPEG } & maleimido-poly-(ethylene & & light chain \\
\hline & glycol) & wt & wild-type \\
\hline PAGE & polyacrylamide electrophoresis & XIAP & X-linked inhibitor of apoptosis \\
\hline PMI & perylene monoimide & & protein \\
\hline PARP & poly (ADP-ribose) polymerase & & \\
\hline PBA & PBS with BSA and azide & & \\
\hline PBS & phosphate buffered saline & & \\
\hline PCR & polymerase chain reaction & & \\
\hline \multirow[t]{2}{*}{ PLAD } & pre-ligand binding assembly & & \\
\hline & domain & & \\
\hline PLGA & polylactide-co-glycoide & & \\
\hline Rel & reticuloendotheliosis oncogene & & \\
\hline RES & reticulo-endothelial system & & \\
\hline rpm & rotations per minute & & \\
\hline RT & room temperature & & \\
\hline
\end{tabular}




\section{Summary}

Polymeric nanoparticles become more and more important as versatile carrier systems for therapeutic and diagnostic compounds embedded within the particle matrix or attached to the particle surface through physical or covalent bonds. Nanoparticle displaying tumor necrosis factor (TNF) on their surface, efficiently activate both TNF receptors and thus mimic the bioactivity of membrane-bound TNF. This leads to a strikingly increased apoptosis. However, an in vivo application for cancer therapy is hampered by the potential systemic action of TNF, which can lead to severe side effects and even death. In this study, targeted lipid-coated TNF-functionalized nanoparticles (scTNF-TLP) were generated, which may be a promising formulation of TNF enabling a systemic and tumor selective application.

ScTNF-TLP are composed of an inner polymeric core with a surface that is functionalized with a single-chain TNF derivative (scTNF). The particles are surrounded by a sterically stabilized polyethylene glycol (PEG)-lipid coat. Thus a strong reduction of the cytotoxic effect could be achieved which indicates an effective shielding of the TNF activity. By insertion of a single-chain $\mathrm{Fv}$ fragment ( $\mathrm{scFv}$ ) directed against the tumor stroma marker fibroblast activation protein (FAP) an active targeting could be demonstrated. The insertion of the targeting moiety into the lipid coat was achieved by a defined and site-directed coupling through a genetically engineered cysteine residue. In order to improve the scFv format for coupling a comparative analysis of various newly designed variants was performed. The most suitable variants contain the hexahistidyl-tag for purification and detection incorporated into the linker sequence together with a cysteine residue (LCH). The resulting TLP and scTNF-TLP bound specifically to FAP-expressing cells but not to FAP-negative cells. The lipid-coating strongly reduced the unspecific uptake of particles and also the scTNF-P mediated apoptosis. In contrast, an increased cytotoxicity towards FAP-expressing cells could be shown for anti-FAP scTNF-TLP compared to lipid-coated scTNF-P without targeting molecule. This indicates a selective delivery of the embedded TNF-functionalized nanoparticle to antigen-positive target cells.

In summary, by encapsulation into a multifunctional lipid-shell, polymeric and TNFfunctionalized nanoparticles, which are subject to an unspecific activity towards a variety of cells and tissue, could be converted in a targeted lipid-coated nanoparticulare carrier system and demonstrated a target cell-specific action of the bioactive compound. This system benefits from a large modularity: beyond the bioactive compound also the targeting moiety as well as the inner core could be adapted at the current requirements. Thus, a manifold application as imaging or drug carrier system is conceivable. Moreover, a combination of both approaches enables usage for a diagnostic as well as therapeutic application. 


\section{Zusammenfassung}

In der letzten Zeit gewinnen polymere Nanopartikel immer mehr an Bedeutung als vielseitige Trägersysteme für therapeutische und diagnostische Komponenten. Diese können innerhalb der Partikelmatrix eingebettet oder auf den Partikeloberflächen mittels chemischer oder physikalischer Verbindung verankert sein. Für Nanopartikel, welche mit dem Tumor Nekrose Faktor (TNF) oberflächenmarkiert sind, konnte eine wirkungsvolle Aktivierung von beiden TNF Rezeptoren gezeigt werden. Sie ahmen somit mebrangebundenes TNF nach. Dadurch wird eine deutliche Zunahme des programmierten Zelltodes, der Apoptose, erzielt. Eine in vivo Anwendung in der Krebstherapie ist jedoch aufgrund des potentiellen systemischen Potentials von TNF nicht möglich. Verabreichung von TNF kann zu massiven Nebeneffekten und sogar zum Tode führen. In dieser Arbeit wurden zielgerichtete lipid-verpackte, mit TNF funktionalisierte Nanopartikel (scTNF-TLP) hergestellt, welche einen vielversprechenden Ansatz für eine systemische und tumor-spezifische Anwendung darstellen.

Ein scTNF-TLP besteht aus einem inneren polymeren Nanopartikel, welcher mit einem einzelkettigen TNF Derivat (scTNF) funktionalisiert ist. Die umgebende Lipidhülle wird mittels Polyethylenglycol (PEG) sterisch stabilisiert. Auf diese Weise wird eine deutliche Reduktion in der Toxizität der scTNF-P erreicht. Dies deutet auf eine effektive Abschirmung der TNF Aktivität hin. Für ein aktives Targeting wurde ein single-chain Fv Fragment (scFv), welches gegen den Tumor Stroma Marker fibroblast activation protein (FAP) gerichtet ist, in die Lipidhülle integriert. Die Insertion der scFv Moleküle erfolgte über eine definierte und zielgerichtete Kopplung mittels eines zusätzlichen, gentechnologisch eingefügten Cysteins. Zur Verbesserung des Formats des scFv Moleküls wurden mehrere neuartige Varianten untereinander verglichen. Als am meisten geeignet erwiesen sich die sog. LCH Varianten, bei denen zusätzlich zum Cystein-Rest, ein zur Aufreinigung und Detektion verwendeter Hexahistidyl-Tag innerhalb der Linkersequenz integriert wurde. Die resultierenden TLP wie auch scTNF-TLP zeigten eine spezifische Bindung an FAP-exprimierende nicht aber an FAP-negative Zellen. Außerdem konnte durch eine Umhüllung mit Lipid die unspezifische Bindung von Partikeln, sowie die von scTNF-Partikeln vermittelte Apoptosis stark reduziert werden. Im Gegensatz dazu wiesen scTNF-TLP eine erhöhte Zytotoxizität für FAP-positive Zellen relativ zu lipid-verpackten scTNF-Partikeln ohne Targeting Molekül auf. Dies spricht für einen selektiven Transport des enthaltenen, mit TNF funktionalisierten Partikels zu Zellen mit dem entsprechenden Antigen.

Es war somit möglich polymere und mit TNF funktionalisierte Nanopartikel, die sich durch eine unspezifische Aktivität gegenüber einer Vielzahl von Zell- und Gewebearten auszeichnen, durch die Verpackung in eine multifunktionale Lipidhülle in ein zielgerichtetes 
lipid-umhülltes nanopartikuläre Trägersystem umzuwandeln. Dieses System zeichnet sich durch eine zielzell-spezifische Wirkung der bioaktiven Komponente aus. Die Vorteile dieses Trägersystems liegen in der großen Modularität: Neben der bioaktiven Komponente können sowohl der innere Kern als auch das Targeting an die jeweiligen Anforderungen angepasst werden. Somit ist eine vielfältige Anwendung in der Bildgebung oder als Trägersystem für Wirkstoffe denkbar. Darüber hinaus kann durch eine Kombination der beiden Ansätze eine gleichzeitige Verwendung im diagnostischen und im therapeutischen Bereich erreicht werden. 


\section{Introduction}

\subsection{The Tumor Necrosis Factor (TNF) and the TNF Receptor Superfamily}

\section{Tumor necrosis factor (TNF)}

At the end of the $19^{\text {th }}$ century, first evidence for the existence of a factor that induces necrosis of tumors was found. The German physician P. Bruns reported a correlation between a bacterial infection and the reduction in tumor mass (Bruns, 1868). Thereupon, the surgeon Coley used bacterial extracts for the treatment of human cancer. In several cases he could observe some tumor regression but the side effects were uncontrollable including even the death of some patients (Coley, 1891). O'Malley et al. (1962) showed that tumor regression is mediated through the induction of a factor in the serum, which they named tumor-necrotizing factor. Old and coworkers renamed it into tumor necrosis factor (TNF) and furthermore identified macrophages as cellular source (Carswell et al., 1975). Between 1984 and 1985 the cDNA of TNF was cloned and the molecule was biochemically characterized (Pennica et al., 1985). Nowadays the structure of human TNF is well known. TNF is a type II transmembrane protein (26 kDa) consisting of 157 amino acids, arranged in stable homotrimers (Jones et al., 1990; Reed et al., 1997), which are held together by hydrophobic interactions. Human TNF is not glycosylated and possesses one disulfide bridge. The membrane bound form of TNF (mTNF) can be proteolytically cleaved at the extracellular region by TACE (metalloprotease TNF alpha converting enzyme, Black et al., 1997) resulting in a soluble form (sTNF, $17 \mathrm{kDa}$ ). TNF is mainly produced by macrophages but also by lymphoid cells, mast cells, endothelial cells fibroblast and neuronal tissue. The soluble as well as the membrane bound form of TNF are able to stimulate cells, whereas MTNF can additionally transfer information between cells, termed juxtatropic signaling.

\section{TNF receptor superfamily}

TNF exerts its biologically function by interaction with members of the so-called TNF receptor (TNFR) superfamily. Until today, 30 receptors are known (Looksley et al., 2001; Branschädel et al., 2007), for instance CD40, Fas (CD95), TRAIL receptors, TNFR1 and TNFR2. Characteristically, receptors of this superfamily contain one to six cysteine-rich domains (CRDs) in their extracellular parts (Naismith \& Sprang, 1998). The two TNF receptors, TNFR1 and TNFR2, each contain four CRDs, a single CRD typically including six cysteineresidues distributed in a range of about 40 amino acids. Furthermore CRD2 and CRD3 were determined to contain the main sites for TNF binding (Banner et al., 1993; Fu et al., 1995). Ligand induced trimerization of the receptors was long considered to be the crucial step for 
signal initiation. However, some years ago, the existence of homotrimers/homodimers for some members of the TNF receptor family in absence of the ligand was shown (Chan et al., 2000; Siegel et al., 2000). The responsible domain, the so-called pre-ligand binding assembly domain (PLAD) was determined to be located within the first CDR and may keep the receptors in a silent status thus preventing an autoactivation observed by receptor overexpression (Wajant et al., 2003). It has been shown that the CDR1 of TNFR1 and TNFR2 contain the PLAD (Chan et al., 2000). CRD1 seem to be also crucial for TNF binding as suggested by molecular dynamics stimulation studies of TNFR1 lacking CRD1. The data indicates that the first region of CRD2 undergoes major conformational changes (Branschädel, 2007).

In general both receptors can be effectively activated by mTNF, whereas only TNFR1 induces intracellular signals after stimulation with sTNF (Grell et al., 1995). Differences in ability to induce complete receptor activation after binding of the membrane bound or the soluble form have also been shown for other members of the TNF receptor superfamily, like TRAIL receptor 2 and Fas (Wajant et al., 2001; Schneider et al., 1998). The stability of the TNF-TNF receptor complexes at $37{ }^{\circ} \mathrm{C}$ has been proposed as rationale for the different signaling capabilities (Grell et al., 1998). It was demonstrated that sTNF dissociates from TNFR1 very slowly $\left(k_{\text {off }}=0.021 \mathrm{~min}^{-1}\right)$, whereas dissociation from TNFR2 sTNF occurs fast $\left(k_{\text {off }}=0.631 \mathrm{~min}^{-1}\right)$. Therefore, binding of sTNF might be too transient for efficient formation of a signaling complex and activation of the TNFR2 pathway might not take place.

\subsection{The TNF Receptor Signal-Transduction Pathways}

The members of the TNF receptor superfamily possess no intrinsic enzymatic activity and thus rely on the recruitment of intracellular adaptor molecules to the cytosolic domain via protein-protein interaction. Two subclasses of receptors regarding their intracellular domains have been defined: the death domain (DD) containing (e.g. TNFR1) and the non-death domain receptors (e.g. TNFR2). TNFR1 is constantly expressed on most cells, whereas TNFR2 is highly regulated and can be found on endothelial and immune cells.

\section{TNFR1 signal-transduction pathways}

The different signaling pathways of TNFR1 correlate with a different subcellular localization of the receptor and the formation of two signaling complexes: complex $I$ is formed at the plasma membrane and induces mostly anti-apoptotic signals, whereas complex II can be found intracellularly and induces apoptosis (Figure 1-1). 


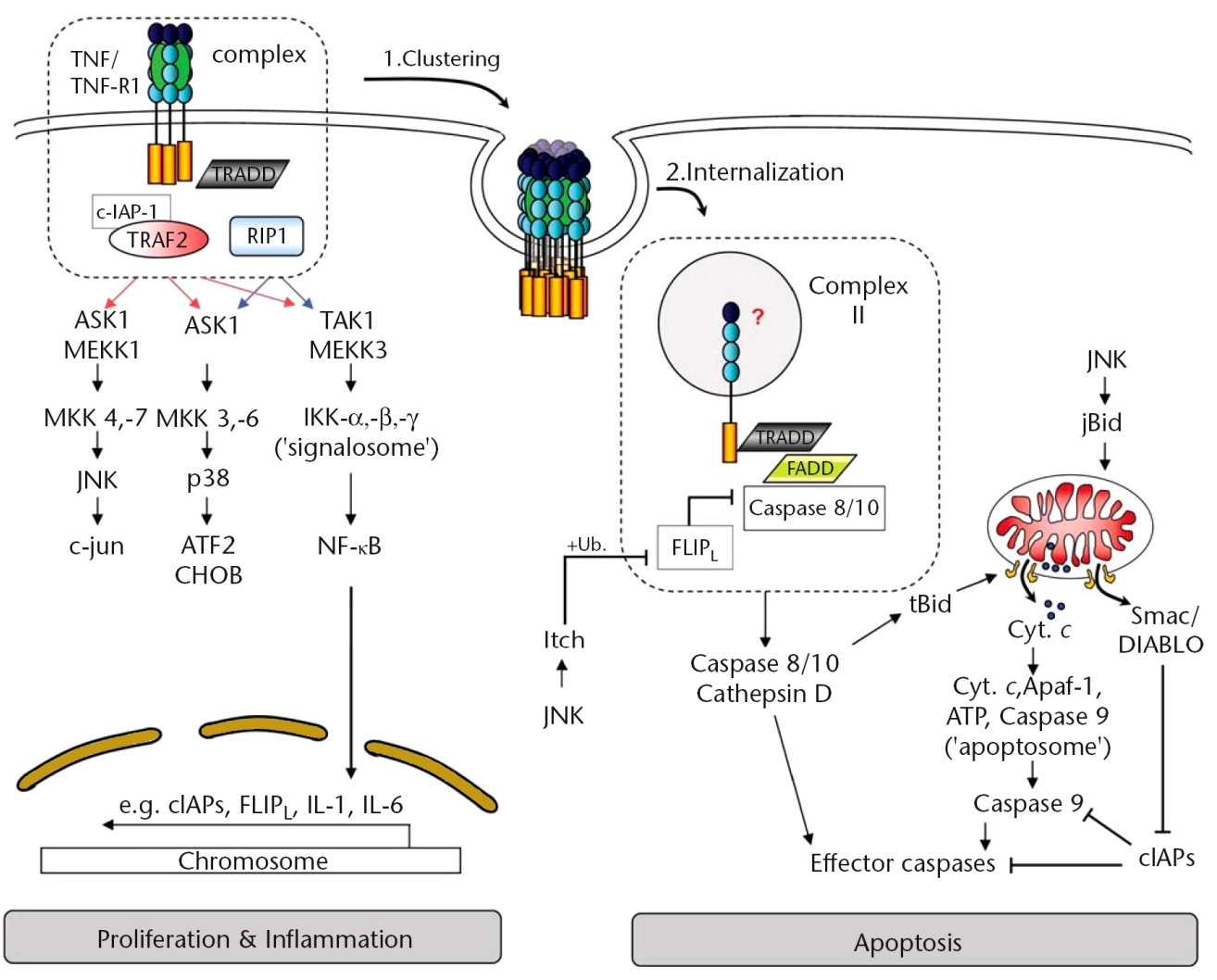

Figure 1-1: Schematic representation of the TNFR1 signaling pathway. Upon binding of TNF (shown in dark green) to TNFR1 an initial protein complex (complex I) is formed at the plasmamembrane leading to the activation of diverse kinases of the MAPK family. Further dowstream kinases of the MAPK cascade become activated, finally resulting in the activation of transcription factors. In addition, the activation of the IKK complex leads to the liberation of the transcription factor NF-KB that translocates into the nucleus to induce gene transcription of several antiapoptotic, proliferative and proinflammatory genes. The formation of the secondary complex (complex II) is shown by the sequential clustering (1) and internalization (2) of TNFR1. The formation of complex II leads to the recruitment of other proteins, such as FADD and caspase 8/10. Effector caspases can be activated by two pathways, either directly by active caspase $8 / 10$ or indirectly by the mitochondrion. The effector caspases accomplish the destruction of the cell. (modified from Branschädel et al., 2007)

Anti-apoptotic signals derive mainly from the activation of the dimeric transcription factor NF$\kappa B$ belonging to the NF-KB/Rel family. The Rel homology domain (RHD) mediates dimerization, DNA binding, nuclear localization and interaction with the inhibitor I-kB (Verma et al., 1995). І-кB interacts with NF-кB dimers to mask their nuclear location sequence, thereby retaining the whole complex in the cytoplasm. Apoptosis is biochemically defined by the activation of caspases. Caspases are zymogens that become active after proteolysis and act themselves proteolytically on other proteins (Riedl \& Shi, 2004). Caspases can be divided into two subfamilies: the initiator caspases and the effector caspases, the latter one become activated by initiator caspases. The spontaneous signaling of TNFR1 without ligand binding is inhibited by silencer of death domains (SODD), which was found to be associated with the DD of TNFR1 (Jiang et al., 1999). TNF treatment releases SODD from the receptor, 
permitting the recruitment of adaptor proteins, like the death domain-containing adapter protein TRADD by homophilic interactions. TRADD serves as an assembly platform for the binding of TNF receptor-associated factor (TRAF) 2 and the death domain-containing serinethreonine kinase receptor-interacting protein RIP (Wajant et al., 2003; Hsu et al., 1996). Subsequently, upstream kinases of the MAPK (mitogen activating protein kinases) family are recruited and activated involving a complex molecular mechanism of phosphorylation and ubiquitination events (Sebban et al., 2006; Kavalenko \& Wallach, 2006). For instance, I-kB is ubiquitinated by the activated I-KB kinase (IKK) complex. Downstream kinases such as MAPK c-Jun N-terminal kinase (JNK) lead to the activation of several transcription factors (Wajant et al., 2003).

The aggregation of TRADD, FADD (Fas-associated death domain), cFLIP (cellular FLICEinhibitory protein) and procaspase-8/10 to establish the TNFR1-associated death inducing signaling complex (DISC) was shown to be dependent on receptor endocytosis (SchneiderBrachert et al., 2004; Micheau \& Tschopp, 2003). Two types of TNFR1 apoptotic signaling are postulated. In type I cells the activation of apoptosis depends on the autoproteolytic cleavage of the procaspases- $8 / 10$ and downstream initiation of effector caspases- 3 and -7 (Shi, 2002). In type II cells, lower levels of activated caspase-8 can be found (Scaffidi et al., 1998). Thus, an additional amplification loop is necessary that involves the cleavage of Bid by caspase- 8 into truncated $(\mathrm{t})$ Bid, mediating cytochrome $\mathrm{c}$ release from mitochondria into the cytosol. This allows the formation of the apoptosome followed by the activation of procaspase-9, which cleaves downstream effector caspases (Wang, 2001). To accomplish apoptosis, the effector caspases then cleave a wide range of defined substrates, for instance poly(ADP)-Ribosme-Polymerase (PARP) (Burkle, 2001), which are responsible for the typical morphological changes observed in apoptotic cells, like membrane blebbing.

\section{TNFR2 signal-transduction pathways}

TNFR2 is a member of non-death domain receptors and contains a TRAF binding site, thus ligand binding induces NF-KB and JNK activation resulting in anti-apoptotic pathways and inflammation (Rothe et al., 1994). Nevertheless it has been observed that selective stimulation of this receptor is sufficient to induce apoptosis in some cells (Medvedev et al., 1994; Grell et al., 1993). In cells, which express both TNFRs, the TNFR2 induced apoptosis is mediated indirectly by the production of endogenous mTNF which has the capability to stimulate TNFR2 in an autocrine dependent manner (Weingärtner et al., 2002). Furthermore, the pro-apoptotic action of TNFR2 relies on the capability to recruit anti-apoptotic protein, like TRAF2. As a consequence of the competition of both TNF receptors for TRAF2, the apoptotic signaling of TNFR1 is enhanced (Fotin-Mleczek et al., 2002). Furthermore, it was 
found that binding of TNF to TNFR2 induces IAP1 (inhibitor of apoptosis) mediated ubiquitination and degradation of TRAF2, which intensifies the TNFR1-induced apoptosis (Li et al., 2002).

\subsection{Cellular and Antitumoral Effects of TNF}

The pleiotropic cytokine TNF can be understood as a proinflammatory molecule that stimulates most cells of the immune system and numerous non-hematopoietic tissues (Figure 1-2). For instance, macrophages secrete TNF upon an infection with gram-negative bacteria, which causes at blood vessels an enhanced blood flow, increases the permeability towards fluids, proteins and production of adhesions molecules, like selectins. This leads to an enhanced adhesion and extravasation of white blood cells.

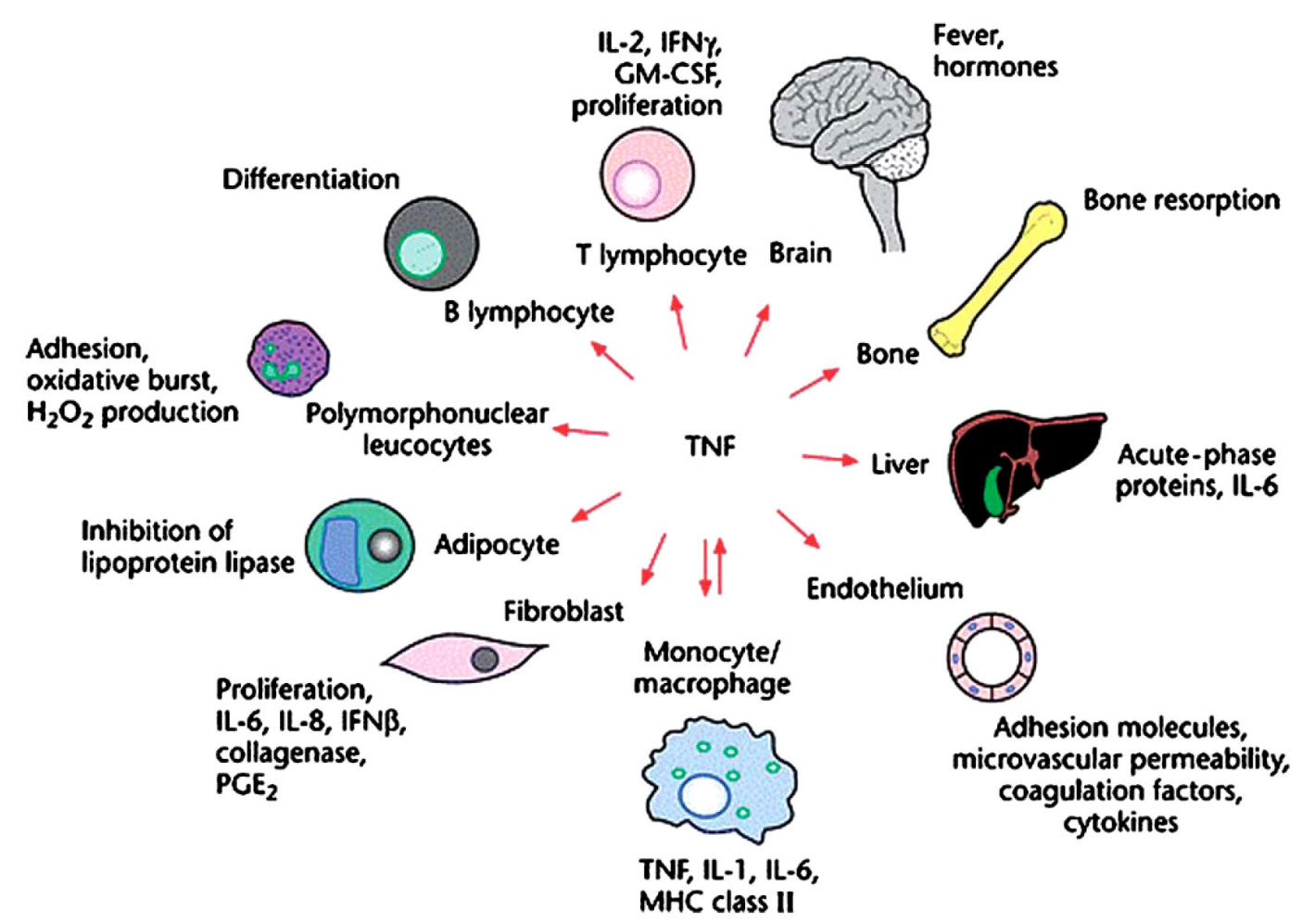

Figure 1-2: Biological activity of tumor necrosis factor (TNF). TNF possesses countless effects on different cell types, thus it is probably the most pleiotropic cytokine. The most important role of TNF is as central regulator of inflammation and immunity. Macrophages are the main producers of TNF, although several cell types can express TNF upon diverse stimuli. II, interleukin; IFN, interferon; GMCSF, granulocyte-macrophage colony-stimulating factor; PGE, prostaglandin E; MHC, major histocompatibility complex (Branschädel et al., 2007).

Furthermore, TNF is essential for the formation of accumulations of macrophages and lymphocytes in granuloma, which prevents spreading of the pathogens like mycobycteria in the organism. However, disregulation in TNF production has been shown to lead to major 
physiological and pathological effects. For instance, raised plasma concentrations of TNF have been detected in patients suffering from bacterial meningitis, cerebral malaria, AIDS and rheumatoid arthritis (Aggarwal, 2003). A definite clinical benefit of anti-TNF strategies has been observed in patients with certain inflammatory autoimmune diseases, like rheumatoid arthritis. Treatment with TNF-specific neutralizing antibodies leads to an improvement of the symptoms (Nash \& Florin, 2005). Nevertheless an unwanted side effect can be the reoccurrence of a latent tuberculosis infection. Although initially thought to be a potent anticancer agent, it is now believed that TNF has limited activity in tumor suppression (Feinberg et al., 1988). Generally, the dosage of administered TNF is limited due to considerable side effects. Some success has been achieved by local administration of TNF via isolated limb and isolated liver perfusion. By means of this technique higher concentrations of TNF can be used without any side effects. Especially in combination with cytostatics like melphalan a high response rate was achieved (Aggarwal, 2003). However this antitumoral activity is probably not due to direct cytotoxic effects of TNF but rather through an action of TNF on the tumor vasculature. This leads to vascular leakage resulting in a good drug distribution all over the tumor (Horssen et al., 2006). An alternative approach to increase efficiency of TNF is the covalent coupling of TNF to solid particles (Bryde et al., 2005, Paciotti et al., 2004). TNF coupled to amino-functionalized silica particles (TNF nanocytes ${ }^{\circledR}$ ) induces a potent activation of TNFR1 as well as TNFR2, initiating strong apoptosis in vitro (Bryde et al., 2005). First in vivo experiments revealed a tumor response upon treatment with silica nanoparticles conjugated either with TNF or in combination with an antibody directed against a tumor specific antigen (Peter Scheurich, personal communication). During the last two decades several approaches of targeted delivery of TNF to tumor cells or to tumor surrounding cells, facilitating effective concentrations at the tumor side by administration of systemically low doses of TNF, have been developed (Gerspach et al., 2009). A limitation of this approach is the affinity of the antibody to its targets, which must be significantly greater than that of TNF to its receptors. Otherwise TNF would not accumulate at the tumor site as required. To circumvent this problem so-called TNF prodrugs are investigated which are fusion proteins consisting of a TNF module, a tumor antigen recognizing domain and a TNF inhibitory domain composed of the extracellular part of TNFR1. The inhibitory domain is released upon processing by tumor specific proteases resulting in active TNF (Gerspach et al., 2009; Gerspach et al., 2006; Wajant et al., 2005). 


\subsection{Single-Chain TNF}

TNF forms stable and tightly packed homotrimers under physiological conditions as mentioned above. It is known that TNF dissociates into its monomers at concentrations below the nanomolar range (Corti et al., 1992) which is commonly reversible. However, TNF is susceptible to hydrophobic interactions, leading to loss of bioactivity and additionally rapid systemic clearance in vivo. Krippner-Heidenreich et al. (2008) constructed a single-chain TNF molecule (scTNF), a TNF mutant consisting of three human TNF monomers fused by two polypeptide linkers composed of serines and glycines (Figure 1-3), thereby preventing a loss in bioactivity caused by dissociation of the trimer.

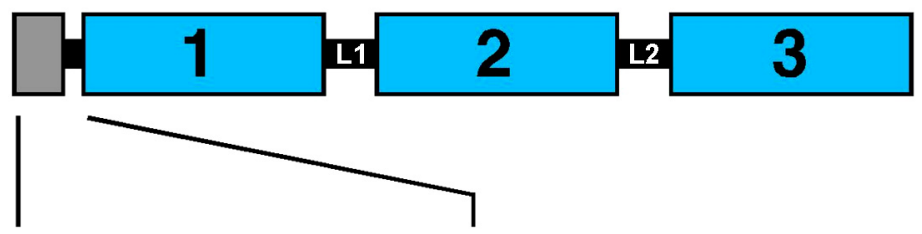

MRGSHHHHHHGSAS-SSSRTPS...

Figure 1-3: Schematic representation of scTNF. The sequence of the $\mathrm{N}$-terminal extension of scTNF is shown, including His-Tag used for purification and detection. TNF modules (blue) are connected by two Glycine-Serine Linkers (L1, L2).

The TNF derivative scTNF revealed a higher stability in vitro and in vivo compared to native sTNF. Binding studies revealed a higher binding affinity of scTNF for both TNF receptors, which could not be displayed in bioactivity assays in cell culture experiments. The bioactivity of scTNF has been determined to be comparable to those of sTNF. Interestingly, scTNF has shown a reduced systemic toxicity and a slightly higher antitumoral activity in two mouse models compared to sTNF. Thus scTNF bears great potential for the generation of new TNFbased therapeutics (Krippner-Heidenreich et al. 2008).

\subsection{Liposomes and Polymeric Nanoparticles}

Liposomes are colloidal, vesicular structures based on lipid bilayers and since they were first described by the British scientist Bangham in 1965 (Bangham et al., 1965) both industry and academia have had high expectations for practical application. The first generation of liposomes, so-called conventional liposomes have been used for the encapsulation of anticancer drugs sometimes resulting in reduced toxicity to normal tissue (Gregoriadis et al., 1971). However, these lipid formulations were rapidly eliminated from the blood circulation by recognition of macrophages of the reticuloendothelial system (RES). To circumvent the short 
half-lives, long-circulating liposomes, so-called "stealth" liposomes, containing inert polymers such as polyethylene glycol (PEG) in the outer lipid layer were established (Allen et al., 1991; Gabizon \& Papahadjopoulos, 1992). The inserted PEG chains prevent the clearance by the RES, resulting in a significant prolongation of circulation of the liposomes. The underlying mechanism may be related to the ability of PEG to form a hydrophilic surface on the liposomes, thus preventing an interaction with plasma proteins, which are involved in the recognition and uptake of liposomes (Allen et al., 1989). Importantly, stealth liposomes have been shown to preferentially accumulate in tumor tissue and therefore reach a higher concentration than in normal tissue. This property is known as the enhanced permeability and retention (EPR) effect also called passive targeting (Maeda, 2001). During angiogenesis tumors release cytokines and other signaling molecules that recruit new blood vessels to the tumor. Angiogenic blood vessels possess larger pore size (200 to $600 \mathrm{~nm}$ ) than observed in vessels of normal tissues; therefore liposomes can extravastate into the interstitial space. Furthermore tumors have only poor lymphatic drainage (Jain, 1987), resulting in an enrichment of liposomes thereby increased drug concentration in tumor tissue relative to administration of the same amount of free drug (Northfelt et al., 1996). The drug uptake has been postulated to be not due to the fusion of liposomes with the cell membrane or by endocytosis but rather by drug release from the liposomes that diffuse though tumor interstitial and passes the cell membrane (Sapra and Allen, 2002). The liposomes serve as a drug reservoir.

Doxorubicin-loaded liposomes (Doxi ${ }^{\circledR} /$ Caelyx $^{\circledR}$, Schering-Plough, Madison NJ, USA) represent an example for a PEGylated liposomal drug carrier system that received clinical approval. Doxil ${ }^{\circledR}$ has been approved for the treatment of AIDS-associated Kaposi's sarcoma (Gottlieb et al., 1997) and ovarian cancer. Liposomal doxorubicin exhibits a prolonged accumulation time in humans and specific enrichment in tumor tissue. Furthermore reduced cardiotoxicity compared to free doxorubicin has been demonstrated. However, side effects like the hand-foot syndrome, a documented side effect for free doxorubicin, have been observed for patients receiving PEGylated liposomal doxorubicin (O'Brien et al., 2004).

The use of polymer-based nanoparticles for drug delivery has been gaining importance and has shown a high therapeutic potential (Lee et al., 2008; Kim et al., 2008). Nanoparticles show a high drug loading capacity with minor drug leakage. Drug release can be manipulated by choosing biodegradable polymers, and external conditions such as $\mathrm{pH}$ and temperature changes may serve as switch for initiating drug release (Rijcken et al., 2007). Furthermore nanoparticles show the ability to circumvent multiple drug resistance compared with reduced toxicity of the drug itself. Additionally, the production of these particles by the miniemulsion method is easy and reproducible (Landfester, 2006) and offers the possibility to 
generate functional groups on particle surface. However, polymeric nanoparticles have shown modest half-lives compared to PEGylated liposomes. One approach to improve biocompatibility and pharmacokinetics of nanoparticles is the encapsulation/coating of particles with PEGylated lipids (Chan et al., 2009; Pulkkinen et al., 2008; van Schooneveld et al., 2008). These core-shell nanoparticles can be used as a platform for therapeutic and imaging modalities. Encapsulation of magnetic particles within liposomes known as magnetoliposomes, provides a promising delivery system, e.g. therapeutic agents, in combination with local hyperthermia (Al-Jamal \& Kostarelos, 2007; Shinkai et al., 2001). In the diagnostic sector, composite silica-nanoparticles have been already described for the design of multifunctional biosensors (Moura et al., 2006; Puu et al., 2006).

\subsection{Immunoliposomes}

As mentioned above, the improved antitumoral activity of liposomes is likely because of the effect called passive targeting. However, despite of the improved circulation properties, an accumulation in liver, spleen and kidney was shown for PEGylated liposomes (Harrington et al., 2001). By a mechanism that is called active targeting, the attachment of peptides, antibodies or antibody fragments on the liposomal surface is thought to increase drug delivery to tumor cells and reduce coincidental accumulation in normal tissues.

Different coupling methods are suitable for the generation of immunoliposomes, for instance the generation of thioether, amide, and hydrazone linkages between antibodies and the liposomal surface (Koning et al., 2003). Immunoliposomes can be grouped into different types depending on the kind of antibody coupling to the liposome surface (Figure 1-4): antibody coupled to the lipid bilayer in the presence or absence of PEG chains (type I $a / b$ ) or to the distal end of a PEG chain incorporated into the lipid bilayer (type II). However, coupling of the antibody to a PEGylated liposomal surface can cause reduction in antigen binding depending on the amount and length of inserted PEG chains (Bendas et al., 1999; Park et al., 1995). These drawbacks can be circumvented by coupling the antibody to the end of a PEG chain (Hansen et al., 1995). 


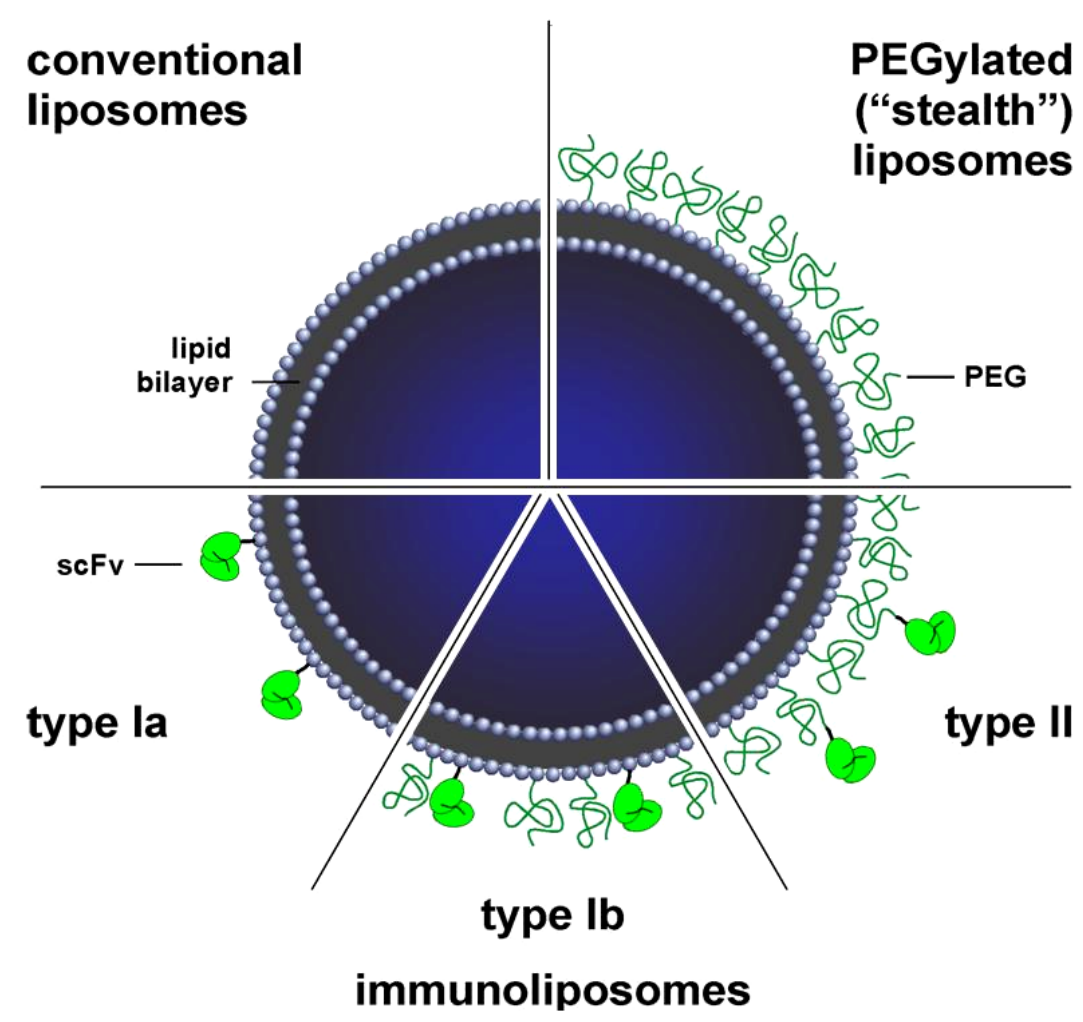

Figure 1-4: Schematic representation of different liposome types. In the upper left site a conventional liposome is presented. On the facing site a sterically stabilized liposome is shown. In the lower part of the figure the different types of immunoliposomes are presented. Type la: antibody (here scFv, green) is coupled to the lipid bilayer; type lb: antibody is coupled to the lipid bilayer of PEGylated liposomes; type II: antibody is coupled to the distal end of PEG chains incorporated into the lipid bilayer

In the beginnings of "active targeting" whole antibodies, mostly monoclonal antibodies of murine origin, have been used for the generation of immunoliposomes. However, these first immunoliposomes were highly immunogenic (Phillips and Dahman, 1995) what prevented a repeated injection (Bendas et al., 2003). Additionally they were rapidly cleared from circulation though recognition of their constant region (Fc) by cells of the RES (Koning et al., 2003). These disadvantages can be principally avoided by the usage of antibody fragments like fragment antigen binding (Fab') or single-chain fragment variable (scFv) fragments.

Fab' fragments are either produced from immunoglobulin (Ig) $G$ molecules by proteolytic cleavage with the enzyme pepsin and a subsequent mild reduction or they are produced in recombinant form for example in bacteria. Immunoliposomes composed of Fab' fragments have been shown to be less immunogenic (Gangné et al., 2002) and show lower clearance from circulation compared to immunoliposomes generated with whole antibodies, probably because the recognition by the RES is circumvented (Pastorino et al., 2003).

Single-chain Fv fragments represent the smallest part of the entire antigen binding site of an antibody in which the variable regions of the heavy and light chain are connected by a 
flexible peptide linker (15 to 20 aa) that further stabilizes the antibody fragment structure (Dübel and Kontermann, 2001). Because of their small size (ca. $25 \mathrm{kDa}$ ) they can be easily produced in bacteria. ScFv fragments can be obtained from hybridoma cells or can be isolated from phage display libraries generated from human donors (Hoogenboom, 2005; Lonberg, 2005). Implementation of human antibody fragments for the generation of immunoliposomes should further reduce immunogenicity. ScFv can be easily genetically modified, for example the introduction of one or more additional cysteine residues (Völkel et al., 2004; Marty et al., 2001) that allow for a site directed coupling to reactive groups. The free sulfhydryl groups of scFv fragments can react with maleimide groups (Mal-PEG) on the liposomal surface, resulting in a stable thioether bond. ScFv-immunoliposomes can be generated by direct conjugation of single-chain Fv (scFv) fragments to Mal-PEG liposomes, called conventional method. In order to use drug loaded immunoliposomes in clinical applications, simple and flexible preparation methods are required. The postinsertion method was first described in 1999 (Ishida et al., 1999). With this method scFv fragments are first coupled to micelles composed of Mal-PEG lipids and then inserted into the outer lipid layer of preformed liposomes in a time and temperature-depended manner. With this method, coupling and drug loading are independent of each other and can be optimized separately. Doxorubicin-loaded sterically stabilized anti-Her2 immunoliposomes (Park et al., 2001) displayed a potent and selective anticancer activity against HER2-overexpressing breast cancer cells and present a well defined targeting-drug system.

By now it is established that the altered antitumoral effect of immunoliposomes compared to non-targeted liposomes is probably not due the increased accumulation in tumor tissues but rather due to internalization of the drug-loaded liposomes as shown in several studies for different immunoliposomes (Kirpotin et al., 2006; Sapra et al., 2004). Interaction of targeted liposomes with cell surface receptors leads to endocytosis of the receptor/immunoliposomes complex. The escape of the entrapped drug from the endosome is mostly required for a successful action and distribution within the cell. Therefore, immunoliposomes may overcome the drug resistance of tumor cells as shown in a study with doxorubicin-loaded immunoliposomes against cancers with low sensitivity to doxorubicin (Hosokawa et al., 2003).

In cancer therapy one has mainly focused on tumor cells as a site of action for immunoliposomes. However, recent focus has been extended to tumor vasculature and tumor stroma as target cells. This approach has several advantages compared to a direct targeting of tumor cells (Augustin, 1998). Tumor endothelial cells are directly accessible for circulating immunoliposomes, which therefore have not to extravastate from the circulation to penetrate into the tumor cells. Furthermore endothelial cells are genetically stable and should 
not develop resistances against therapeutic drugs, as it is observed for the genetically unstable tumor cells. All solid tumor growth depends on neovascularization; targeting and destruction of the vasculature should consequently affect a large number of tumor cells. Fibroblasts, the major cell population in the tumor stroma, contribute to the formation of the tumor stroma by alteration of the extracellular matrix and by promoting tumor cell invasion and metastasis. They also facilitate tumor angiogenesis and availability of signaling molecules like growth factors and cytokines (Kalluri and Zeisberg, 2006). The human fibroblast activation protein (FAP) is a cell surface protein of stromal fibroblasts of tumors and serves as an interesting target in tumor therapy. It is a type II transmembrane protein with an extracellular C-terminal region followed by a single hydrophobic transmembrane domain and a short cytoplasmic tail (Scanlan et al., 1994). FAP belongs to the serine protease family and exhibits dipeptidyl peptidase and also a collagenolytic activity, thereby capable to degrade gelatin I and type I collagen of the extracellular matrix (Park et al., 1999). Therefore it is hypothesized that FAP possesses a role in the remodeling of tumor tissue, for instance influencing invasion and metastasis. FAP forms homodimers with a monomeric molecular mass of $95 \mathrm{kDa}$. It is well expressed by reactive stromal fibroblast of human epithelial cancers in more than $90 \%$ of breast, lung, colorectal and ovary carcinomas. Expression of FAP in normal tissues has only been transiently seen in healing wounds and by the fetus (Ramirez-Montagut et al., 2004; Rettig et al., 1993; Garin-Chesa et al., 1990). Several specific antibodies against FAP have been isolated by phage display or humanized by guided selection (Brocks et al., 2001; Mersmann et al., 2001; Schmidt et al., 2001). The antibody fragment generated by Brooks, named ScFv MO36 was used for the generation of targeted delivery systems in this study. ScFv MO36 recognizes human and murine FAP which share $87 \%$ homology (Niedermeyer et al., 1997).

\subsection{Aim of the Study}

The aim of the study was the generation of a safe and efficient formulation of TNF that should enable a systemic application and induction of tumor selective activity. Therefor, the advantages of complex polymer nanoparticles and liposomes should be combined by the implementation of multifunctional TNF-functionalized, lipid-coated nanoparticle composite systems. As model system polystyrene-based nanoparticles coated with a single-chain TNFfunctionalized surface were used, which were coated with a sterically stabilized PEG-lipid shell. The lipid coating is thought to reduce strongly the cytotoxic effect on target-negative cells, verifying effective shielding of TNF activity. Furthermore, the implementation of a targeting moiety should mediate selective delivery of the embedded TNF-functionalized 
nanoparticle to antigen-positive target cells.

For this purpose a new method had to be established for the coating of the nanoparticles with a sterically stabilized PEG-lipid shell. By insertion of single-chain Fv-PEG-lipids into the lipid coat the PEG-lipid shell should become endowed with a targeting moiety. As target antigen the fibroblast activation protein (FAP) was used exemplary. 


\section{Materials and Methods}

\subsection{Materials}

\subsubsection{Instruments and Special Implements}

Balances

Blotter

Centrifuges

Electrophoresis System / Power Supply

\section{ELISA Plate Reader}

Extruder

Film developing machine

Flow Cytometer

Gel documentation

Heat block

HPLC System

Incubator for bacteria

Incubator for cell culture

LI-COR

Magnetic stirrer

Microscope
Feinwaage Basic [Sartorius AG, Göttingen, Germany] 440-39N and 440-33N [Kern, Balingen, Germany]

TransBlot SD, semidry transfer cell [Bio-Rad, Munich, Germany]

Eppendorf 5415D, 5415C and 5415 R [Eppendorf, Hamburg, Germany]

Eppendorf 5810R (cell culture) [Eppendorf, Hamburg, Germany] J2-MC with Rotors JA10, JA14, JA20 [Beckman Coulter, Krefeld, Germany]

Avanti J-30I [Beckman Coulter, Krefeld, Germany] ultracentrifuge Optima TL [Beckman Coulter, Krefeld, Germany] L7 ultracentrifuge [Beckman Coulter, Krefeld, Germany]

Mini-PROTEAN 3 Electrophoresis Cell System [BioRad, Munich, Germany]

Ready Agarose Precast Gel System [BioRad, Munich, Germany]

Power Pac Basic [BioRad, Munich, Germany]

SPECTRAmax 340PC Microplate Spectrophotometer [Molecular Devices, Palo Alto, USA]

LiposoFast. Basic [Avestin, Ottawa, Ca]

X-OMAT 1000 Processor [Kodak, Rochester, USA]

EPICS XL-MCL Flow Cytometer [Beckman Coulter, Krefeld, Germany]

Cytomics FC 500 [Beckman Coulter, Krefeld, Germany]

Transilluminator, Gel documentation system Felix [Biostep, Jahnsdorf, Germany]

HBT-1-131, [Hlc-Haep Labor Consult, Bovenden, Germany]

Waters HPLC-System [Millipore, Billerica, USA], Chromatography Software Clarity Lite v.2.4.1.65

BD 53, [Binder, Tuttlingen, Germany] Infors HAT Multitron 2, [Infors Ag, Basel, $\mathrm{CH}$ ]

$\mathrm{CO}_{2}$ Inkubator [Zapf, Sarstedt, Germany]

Odyssey [LI-COR Bioscience, Bad Homburg, Germany] MR $3001 \mathrm{~K} 800 \mathrm{~W}$, [Heidolph Instruments, Nürnberg, Germany] Olympus CK2 [Olympus, Hamburg, Germany]

Leica DM IRB with Zeiss AxioCam MRc5 [Wetzlar/Oberkochen, Germany] Software AxioVision v4.5 
PCR cycler

Spectrophotometer

Sterile bench

Tecan

Vortexer

Water bath

ZetaSizer
Cellobserver [Carl Zeiss Microlmaging GmbH, Jena, Germany] RoboCycler 96 [Stratagene, La Jolla, USA]

GeneQuant (260 nm/280 nm), Ultrospec 1000 (600 nm) [Pharmacia Biotech, Uppsala, S]

Sicherheitswerkbank Variolab Mobilien W90,

[Waldner-Laboreinrichtungen, Wangen, Germany]

Tecan infinite M200 [Tecan Austria, Grödig, Austria]

Sky Line, [Elmi Ltd., Riga, Latvia]

MA6, [Lauda, Lauda-Königshofen, Germany]

$\mathrm{GFL}^{\circledR}$

ZetaSizer Nano ZS [Malvern Instruments, Herrenberg, Germany]

\section{Special Implements and Consumables}

Cover slip

Dialysis membrane

cover slip $\varnothing 22$ mm [Roth, Karlsruhe, Germany]

ZelluTrans MWCO 8000-10000 [Carl Roth, Karlsruhe, Germany]

Dialysis tubes

D-Tube ${ }^{\mathrm{TM}}$ Dialyzer Mini, MWCO 6-8 kDa [CalbiochemVovabiochem, Läufelfingen, Germany]

FACS tubes

HPLC column

IMAC affinity matrix

Microscope slide

Microtiter plate

Nitrocellulose membrane

FACS tubes PS, 5ml [Greiner Bio-One, Frickenhausen, Germany]

BioSep-SEC-S2000 or S3000 [Phenomenex, Aschaffenburg, Germany]

Ni-NTA-Agarose [Qiagen, Hilden, Germany]

glass slide 76x26 mm [Roth, Karlsruhe, Germany]

Microtiter plate Cellstar 96 well (V-bottom/ F-bottom) [Greiner Bio-One, Frickenhausen, Germany]

BioTrace NT Nitrocellulose Transfer Membrane [Pall Life Sciences; East Hills, USA]

Polycarbonate filter membrane

Armatis (pore diameter: $50 \mathrm{~nm}$; diameter: $19 \mathrm{~mm}$ ) Liposofast [Avestin, Ottawa, Canada]

Sepharose

Sepharose $^{\mathrm{TM}}$

München,Germany]

4B [Amersham-Biosciences,

cut-off $0.2 \mu \mathrm{m}$ (FP30/0,2 CA-S) and cut-off $0.45 \mu \mathrm{m}$ (FP30/0,45 CA-S), cellulose acetate, non-pyrogenic [Schleicher \& Schuell, Brentford, UK]

Tissue culture flasks and CellStar [Greiner Bio-One, Kremsmünster, Austria] dishes

Ultracentrifuge tubes

ultracentrifuge tubes $9 / 16 \times 31 / 2$, [Beckman Coulter, Krefeld, Germany]

Whatman Filter Paper 
Brentford, UK]

X-Ray film

Medical X-Ray film 100 NIF 18 x 23 cm [Fuji, Düsseldorf, Germany]

\subsubsection{Chemicals and Lipids}

BioRad Protein Assay was purchased from BioRad Laboratories GmbH [Krefeld, Germany]. TCEP BondBreaker ${ }^{\mathrm{TM}}$ Solution (\#77720) was purchased from Pierce [Rockford, USA]. Collagen $\mathrm{R}$ was purchased from Serva Electrophoresis $\mathrm{GmbH}$ [Heidelberg, G]. All other chemicals were purchased from Roth [Karlsruhe, Germany], Sigma-Aldrich [St. Louis, USA], Merck [Darmstadt, Germany] and Roche [Basel, Switzerland] unless otherwise stated and had a purity $\geq 99 \%$.

\section{Lipids}

Cholesterol

highly purified

Calbiochem, Darmstadt,

Dil

1,1'-dioctadecyl-3,3,3',3'Germany tetramethylindocarbo Sigma-Aldrich, St. Louis, cyanine perchlorate

DiO 3,3'-dioctadecyloxacarbocyanine perchlorate

Sigma-Aldrich, St. Louis, USA

EPC

egg phosphatidylcholine

Lipoid, Ludwigshafen,

Mal-PEG ${ }_{2000}$-DSPE 1,2-distearoyl-sn-glycero-3Germany

phosphoethanolamine- $\mathrm{N}$ Avanti Polar Lipids, [maleimide(polyethylene glycol)-2000] Alabaster, USA (ammonium salt) $\mathrm{mPEG}_{2000}$-DSPE 1,2-distearoyl-sn-glycero-3Avanti Polar Lipids, phosphoethanolamine- $\mathrm{N}$ Alabaster, USA [methoxy(polyethylene glycol)-2000] (ammonium salt) 


\subsubsection{Cell lines}

Table 1 Cell lines and corresponding culture conditions.

\begin{tabular}{|c|c|c|c|c|c|}
\hline Cell line & origin & $\begin{array}{l}\text { stably } \\
\text { transfected }\end{array}$ & culture & media & selection \\
\hline $\begin{array}{l}\text { HT1080 } \\
\text { wt }\end{array}$ & $\begin{array}{l}\text { human } \\
\text { fibrosarcoma }\end{array}$ & & adherent & $\begin{array}{l}\text { RPMI } 1640+ \\
5 \% \text { FCS }\end{array}$ & \\
\hline $\begin{array}{l}13.8\left(\mathrm{FAP}_{\mathrm{mo}}\right) \\
33\left(\mathrm{FAP}_{\mathrm{hu}}\right)\end{array}$ & & $\begin{array}{l}\text { mouse FAP } \\
\text { human FAP }\end{array}$ & & & $\begin{array}{l}\text { G418 } \\
\text { G418 }\end{array}$ \\
\hline Kym1 & $\begin{array}{l}\text { human } \\
\text { rhabomyosarcoma }\end{array}$ & & adherent & $\begin{array}{l}\text { RPMI } 1640+ \\
10 \% \text { FCS }\end{array}$ & \\
\hline
\end{tabular}

Cells were cultured at $37{ }^{\circ} \mathrm{C}$ in a humidified (60\% rel. humidity) incubator with a $5 \% \mathrm{CO}_{2}$ atmosphere.

\subsubsection{Bacterial Strain E. coli TG1}

Genotype: supE thi-1 $\Delta($ lac-proAB $) \Delta($ mcrB-hsdSM $) 5\left(\mathrm{r}_{\mathrm{K}}{ }^{-} \mathrm{m}_{\mathrm{K}}{ }^{-}\right)\left[\mathrm{F}^{\prime}\right.$ traD36 proAB laclqZ $\left.\Delta M 15\right]$ [Stratagene, La Jolla, USA]

\subsubsection{Media and Supplements}

\section{Bacterial culture}

LB-Medium, 1x

TY-Medium, 2x

LB $_{\text {Amp, Glc }}$ Agar plates

IPTG

Ampicillin

\section{Cell culture}

RPMI 1640

FCS (Fetal calf serum)

Trypsin/EDTA, 10x

Penicillin/Streptomycin

(P/S), 100x

Geneticin (G418)

Eosin
$1 \%$ peptone, $0.5 \%$ yeast extract, $0.5 \% \mathrm{NaCl}$ in $\mathrm{H}_{2} \mathrm{O}$

$1.6 \%$ peptone, $1 \%$ yeast extract, $0.5 \% \mathrm{NaCl}$ in $\mathrm{H}_{2} \mathrm{O}$

LB-Medium, $1.5 \%$ agar, autoclave, add $100 \mu \mathrm{g} / \mathrm{ml}$ ampicillin, $1 \%$ glucose after cooling-down

Isopropyl- $\beta$-D-thiogalactopyranoside, stock solution $1 \mathrm{M}$ in $\mathrm{H}_{2} \mathrm{O}$ [Gerbu Biochemicals, Gaiberg, Germany]

$100 \mathrm{mg} / \mathrm{ml}$ in $\mathrm{H}_{2} \mathrm{O}$ [Roth, Karlsruhe, Germany]

+ $2 \mathrm{mM}$ glutamine [Gibco/Invitrogen, Carlsbad, USA]

FBS Standard Quality, EU approved, Cat \#A15-101, Lot \#A10106-1033 [PAA Laboratories, Pasching, Austria]

Heat inactivated at $56{ }^{\circ} \mathrm{C}$ for 30 minutes

$0.5 \%$ Trypsin, $5.3 \mathrm{mM}$ EDTA, diluted to $1 \mathrm{x}$ in PBS [Gibco/Invitrogen, Carlsbad, USA]

$10^{4} \mathrm{U} / \mathrm{ml} / 10^{4} \mu \mathrm{g} / \mathrm{ml}$ [Gibco/Invitrogen, Carlsbad, USA]

$100 \mathrm{mg} / \mathrm{ml}$ in PBS [Gibco/lnvitrogen, Carlsbad, USA]

$0.4 \%$ Eosin, $10 \%$ FCS, $0.02 \% \mathrm{NaN}_{3}$ in sterile PBS 


\subsubsection{Solutions}

Blotting buffer, $1 \mathrm{x}$

$20 \%$ methanol, $192 \mathrm{mM}$ glycine, $25 \mathrm{mM}$ Tris, $\mathrm{pH} 8.3$

Bradford Solution

Bio-Rad Protein Assay [Bio-Rad, Munich, Germany]

Coomassie destain solution

$45 \%$ methanol, $10 \%$ glacial acetic acid, $45 \% \mathrm{H}_{2} \mathrm{O}$

Coomassie solution

$0.25 \%$ Coomassie Blue R250 in Coomassie destain solution

Coupling buffer

$10 \mathrm{mM} \mathrm{Na} \mathrm{HPO}_{4} / \mathrm{NaH}_{2} \mathrm{PO}_{4}$ buffer, $0.2 \mathrm{mM}$ EDTA, 30 $\mathrm{mM} \mathrm{NaCl}, \mathrm{pH} 6.7$

DNA loading buffer, $5 x \quad 1 \mathrm{ml} 50 x$ TAE buffer, $2.5 \mathrm{ml}$ glycerol, $0.02 \%$ (w/v) bromophenol blue, ad $10 \mathrm{ml} \mathrm{H}_{2} \mathrm{O}$

ECL reagent Solution A $\quad 0.1 \mathrm{M}$ Tris, 1.25 mM luminol sodium salt in $\mathrm{H}_{2} \mathrm{O}, \mathrm{pH} 8.6$

ECL reagent Solution $B$ $11 \mathrm{mg} \mathrm{p}$-Coumaric acid in $10 \mathrm{ml}$ DMSO

IMAC Na-phosphate buffer, $250 \mathrm{mM}$ Na-phosphate (37.38 g Na $\mathrm{HPO}_{4} \cdot 2 \mathrm{H}_{2} \mathrm{O}$ + $5 x$ (low salt)

$\left.6.24 \mathrm{~g} \mathrm{NaH}_{2} \mathrm{PO}_{4} \cdot 2 \mathrm{H}_{2} \mathrm{O}\right), 1.25 \mathrm{M} \mathrm{NaCl}, \mathrm{pH} 7.5$, ad 1 I $\mathrm{H}_{2} \mathrm{O}$

L-cysteine $\quad 1 \mathrm{mM}$ L-cysteine, $0.02 \mathrm{mM}$ EDTA, pH 5.5

PBA

PBS (1x)

Periplasmatic protein

preparation buffer 1xPBS, $0.02 \%$ Na-acid, $2 \%$ FCS

SDS running buffer, $10 \mathrm{x}$

$2.67 \mathrm{mM} \mathrm{KCl}, 1.47 \mathrm{mM} \mathrm{KH}_{2} \mathrm{PO}_{4}, 137.93 \mathrm{mM} \mathrm{NaCl}$, $8.06 \mathrm{mM} \mathrm{Na}_{2} \mathrm{HPO}_{4} \cdot 7 \mathrm{H}_{2} \mathrm{O}, \mathrm{pH} 7.5$

TAE buffer, $50 x$

$30 \mathrm{mM}$ Tris- $\mathrm{HCl} \mathrm{pH}$ 8.0, 1 mM EDTA, $20 \%$ sucrose in $\mathrm{H}_{2} \mathrm{O}$

1.92 M glycine, $0.25 \mathrm{M}$ Tris, 1 \% SDS, pH 8.3

$2 \mathrm{M}$ Tris, $0.95 \mathrm{M}$ glacial acetic acid, $50 \mathrm{mM}$ EDTA in $\mathrm{H}_{2} \mathrm{O}, \mathrm{pH} 8$

\subsubsection{Antibodies, Enzymes, Kits, Markers etc.}

\section{Antibodies}

His-Probe-HRP [sc-8036 HRP, Santa Cruz 1:1000 (western Blot)

Biotechnology, Santa Cruz USA]

Alexa Fluor 848 anti human CD105 antibody $7 \mu \mathrm{l} / 10^{6}$ cells (immuno [Biozol, Eching, Germany] fluorescence)

anti-(His) $)_{6}$-Tag-FITC, murine IgG1, DIA920 1:500 (flow cytometry)

[Dianova, Hamburg, Germany]

\section{Enzymes}

Alkaline phosphatase

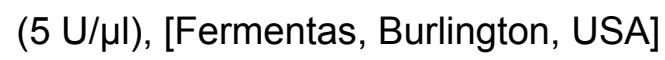

REDTaq ReadyMix ${ }^{\mathrm{TM}}$

$(0,06 \mathrm{U} / \mu \mathrm{l})$, [Sigma-Aldrich, St. Louis, USA] 
T4 DNA Ligase

Taq DNA-Polymerase

\section{Restriction enzymes}

EcoRI

Notl

Sfil

Xhol

Kits

NucleoSpin® Extract II Kit [Macherey-Nagel, Düren, Germany]

PureLinkTM HiPure Plasmid MidiPrep Kit [Invitrogen, San Diego, USA]

\section{Marker}

Gene RulerTM DNA Ladder Mix ready-to-use \#SM0333 [Fermentas, St. Leon-Rot, Germany] Page RulerTM Prestained Protein Ladder \#SM0671 [Fermentas, St. Leon-Rot, Germany]

\subsubsection{Primers}

Primers were purchased from Metabion, Martinsried, Germany.

\section{Primers for screening or sequencing}

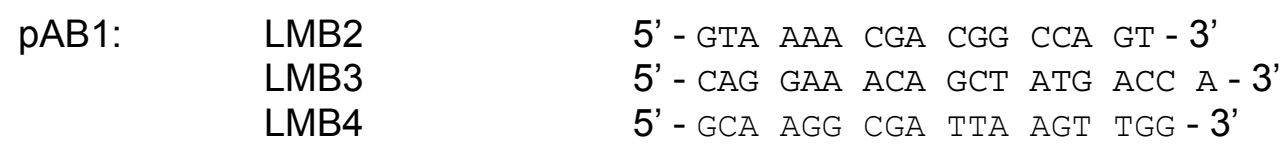

\section{Primers for amplification of scFv`36 HC2, scFv`36 HC3, scFv`HC4} HC2-EcoRI-For

\footnotetext{
5- GCG CAT CAT CAC CAT CAC CAT GGC GGA TCG AGT GGC TCA GGA TGC TAA GAA TTC CAC TGg 3-
}

\section{HC3-EcoRI-For}

$\begin{array}{lllllllllllllllllllllllllllll}\text { A } & \text { H } & \text { H } & \text { H } & \text { H } & \text { H } & \text { H } & \text { G } & \text { G } & \text { S } & \text { S } & \text { G } & \text { S } & \text { C } & \text { G } & \text { C } & \text { S } & \text { C } & -\end{array}$

5- GCG CAT CAT CAC CAT CAC CAT GGC GGA TCG AGT GGC TCA TGC GGA TGT AGT TGC TAA GAA TTC CAC TGG 3-

\section{HC4-EcoRI-For}

5- CAT CAT CAC CAT CAC CAC GGC GGA TCC AGC GGC GGA TCC AGC GGC TCC GGA TGC TAA GAA TTC CGG 3-

Primers for amplification of scFv`36 LC1, scFv`36 LC2, scFv`LC3

\section{LC1-Xhol-Back}

5- ACC GTC TCG AGT TGC GGA GGC GGT TCA GGC GGA GGT GGC TCT 3- 


\section{LC2-Xhol-Back}

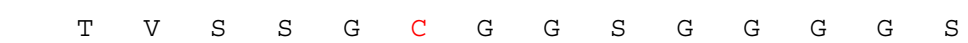

$5^{-}$ACC GTC TCG AgT GGT TGC GGC GGT TCA GGC GGA GGT GGC TCT 3-

LC3-Xhol-Back

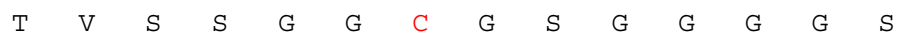

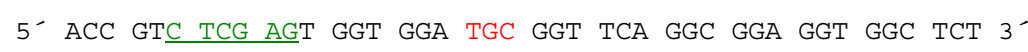

Primers for amplification of scFv`36 LCH1, scFv`36 LCH3

LHC1-Xhol-Back

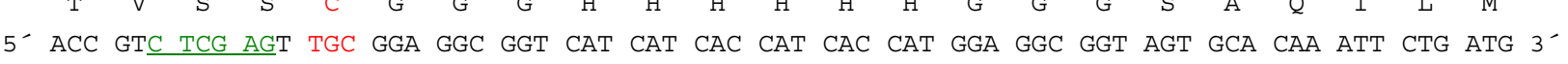

\section{LHC3-Xhol-Back}

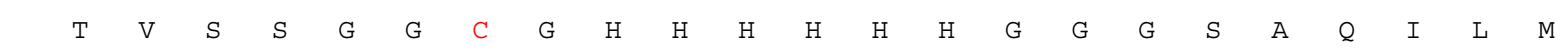
5- ACC GTC TCG AGT GGT GgA TGC GGT CAT CAT CAC CAT CAC CAT GgA GgC GGT AGT GCA CAA ATT CTG ATG 3-

\section{stop-EcoRI-For}

$5^{-}$GGG ACC AAg CTG GAA ATA AAA CGg tAA GAA TTC ACT GGC 3-

\subsubsection{Vectors}

The vector pABC4 (3312 bp) for prokaryotic protein expression derives from pAB1. In pABC4 the Myc-tag was removed and an additional cysteine residue added behind the Histag.

pAB1: Vector for prokaryotic periplasmic protein expression in E. coli TG1 (Kontermann et al., 1997). 
a)

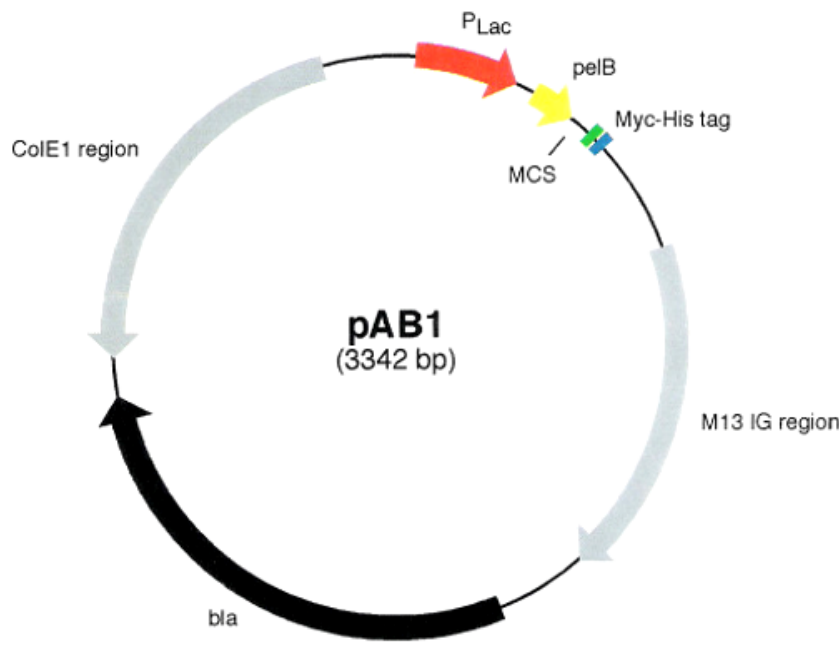

b)

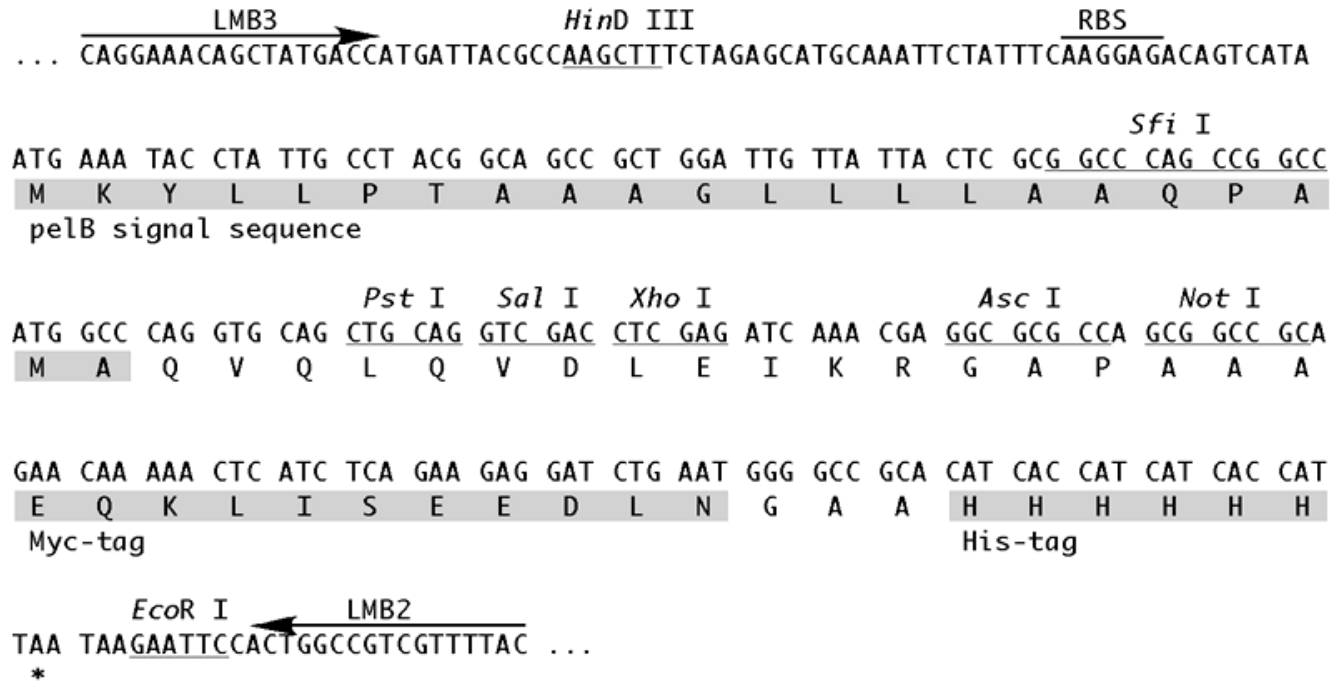

Figure 2-1: (a) Map of vector $p A B 1: P_{L a c}$ : lacl repressor and lacZ promotor, pelB: signal sequence for periplasmatic secretion, MCS: multiple cloning site, Myc- and His-tag, M13 IG region: intergenic region, bla: $\beta$-lactamase (ampicillin resistance), ColE1: origin of replication (b) Section of pAB1 sequence indicating peIB signal sequence for secretion, the Myc- and His-tag, multiple cloning sites and primer sequences LMB3/LMB2. 


\subsection{Methods}

\subsubsection{Cloning of scFv' variants}

\subsubsection{Cloning strategies for the scFv' constructs}

scFv'36 HC variants

The scFv'36 constructs (HC2-4) were amplified from the vector pAB1 scFv'36 with the primers LMB3 and HC2-EcoRI-For, HC3-EcoRI-For or HC4-EcoRI-For, respectively. The $\mathrm{PCR}$ products were digested with Sfil and $\mathrm{EcORI}$ and cloned into vector pABC4 digested with the same enzymes.

scFv'36 LC variants

For generating the LC variants (LC1-3), scFv' molecules were amplified from pAB1 scFv36 with the primers LMB2 and LC1-Xhol-Back, LC2-Xhol-Back or LC3-Xhol-Back, respectively. PCR products were digested with Xhol and Notl and cloned into pAB1 scFv36 digested with the same enzymes.

scFv'36 LCH variants

The both LCH constructs ( $\mathrm{LCH} 1$ \& 3) were amplified from pAB1 scFv36 with the primers stop-EcoRI-For and LCH1-Xhol-Back or LCH3-Xhol-Back, respectively. PCR products were digested with Xhol and EcoRI and cloned into pAB1 scFv36 digested with the same enzymes.

\subsubsection{Polymerase Chain Reaction (PCR)}

Polymerase Chain Reaction was used to amplify desired DNA fragments out of the corresponding vectors. Thereof, a PCR reaction mix was prepared:

$\begin{array}{ll}\text { DNA template }(1 \mathrm{ng} / \mu \mathrm{l}) & 10 \mu \mathrm{l} \\ 10 x \text { buffer with }\left(\mathrm{NH}_{4}\right)_{2} \mathrm{SO}_{4} & 5 \mu \mathrm{l} \\ \mathrm{MgCl}_{2}(25 \mathrm{mM}) & 4 \mu \mathrm{l} \\ \text { Forward primer }(10 \mathrm{pmol} / \mu \mathrm{ll}) & 1 \mu \mathrm{l} \\ \text { Reverse primer }(10 \mathrm{pmol} / \mu \mathrm{l}) & 1 \mu \mathrm{l} \\ \text { dNTP }(20 \mathrm{mM}) & 2.5 \mu \mathrm{l} \\ \text { Taq DNA-Polymerase }(1 \mathrm{U} / \mu \mathrm{l}) & 1.25 \mu \mathrm{l} \\ \mathrm{dH}_{2} \mathrm{O} & \text { ad } 50 \mu \mathrm{l}\end{array}$

The amplification was performed using the following PCR program: 


\begin{tabular}{ll|l}
$5 \min$ & $94^{\circ} \mathrm{C}$ & \\
$1 \min$ & $94^{\circ} \mathrm{C}$ & \\
$1 \min$ & $50{ }^{\circ} \mathrm{C}$ & $30 \mathrm{x}$ \\
$1 \mathrm{~min}$ & $72{ }^{\circ} \mathrm{C}$ & \\
$5 \mathrm{~min}$ & $72{ }^{\circ} \mathrm{C}$ & \\
forever & $4{ }^{\circ} \mathrm{C}$ &
\end{tabular}

The successful amplification was controlled on a $1.0 \%$ agarose gel and the PCR product isolated by agarose gel electrophoresis followed by DNA gel extraction (NucleoSpin Extract II, PCR Clean-up Gel extraction kit, Macherey-Nagel) (2.2.1.4).

\subsubsection{Restriction Digestion}

Ten $\mu \mathrm{g}$ vector DNA or total amount of DNA extracted from agarose gel were digested in a total volume of $50 \mu \mathrm{l}$. Restriction enzymes (Fermentas) were added ( $20 \mathrm{U} /$ reaction) and incubated with the corresponding buffers and conditions according to the manufacturer's protocol for $3 \mathrm{~h}$. For buffer exchange the NucleoSpin Extract II, PCR Clean-up Gel extraction kit (Macherey-Nagel) was used.

To avoid vector religation, digested vector DNA was dephosphorylated after restriction digestion by adding $1 \mathrm{U}$ alkaline phosphatase to the reaction mix and incubated for $1 \mathrm{~h}$ at 37 ${ }^{\circ} \mathrm{C}$.

\subsubsection{Agarose Gel Electrophoresis and DNA Gel Extraction}

Analysis and purification of DNA (amplified or digested DNA) was performed by horizontal agarose gel electrophoresis. DNA samples were mixed with 5x DNA loading buffer and separated using a $1.0 \%$ agarose gel containing $1 \mu \mathrm{g} / \mathrm{ml}$ ethidium bromide in TAE buffer. Samples were run at $85 \mathrm{~V}$ for 60 minutes. Relevant DNA bands were excised under UV light and extracted with a DNA gel extraction kit (NucleoSpin Extract II, PCR Clean-up Gel extraction kit, Macherey-Nagel) according to the manufacturer's protocol. DNA was eluted in $30 \mu \mathrm{H}_{2} \mathrm{O}$.

\subsubsection{Ligation}

Ligation of linearized, dephosphorylated vector and insert at a molar ratio of 1:3 to 1:5 was carried out with T4 DNA ligase $(5 \mathrm{U})$ in ligation buffer in a total reaction volume of $20 \mu \mathrm{l}$. After incubation for $1 \mathrm{~h}$ at room temperature, $10 \mu \mathrm{l}$ of the ligation mixture were used for the transformation of chemical competent $E$. coli TG1 cells. 


\subsubsection{Transformation of E. coli TG1}

$100 \mu \mathrm{l}$ of chemical competent E. coli TG1 cells were thawed on ice and mixed with $10 \mu \mathrm{l}$ of ligation mixture. After 15 minutes incubation on ice, the cells were subjected to a heat shock at $42{ }^{\circ} \mathrm{C}$ in a water bath for 45 seconds and cooled down on ice for 1 minute. $1 \mathrm{ml}$ of LB medium was added and the cells were incubated for $1 \mathrm{~h}$ at $37{ }^{\circ} \mathrm{C}$ on a shaker to allow the expression of the resistance protein. The cells were harvested (1 min, $16000 \mathrm{~g}$ ), plated on $\mathrm{LB}_{\mathrm{Amp}, \mathrm{Glc}}$ plates and incubated over night at $37^{\circ} \mathrm{C}$.

\subsubsection{Screening of Clones}

Single colonies were picked and the incorporation of DNA insert was checked by screening PCR using the REDTaq ReadyMix PCR Reaction Mix (Sigma-Aldrich). Simultaneously, the same colonies were plated on a master plate and incubated over night $\left(37^{\circ} \mathrm{C}\right)$. The primers were chosen according to the expected DNA fragments and plasmids to obtain an unambiguous PCR result. The PCR fragments were separated by agarose gel electrophoresis and positive clones with correct DNA integration were identified due to bands of predicted size. One positive clone was used for an over night culture for plasmid DNA isolation (Midi preparation).

\subsubsection{Plasmid-DNA Isolation (Midi)}

For the isolation of plasmid DNA, a positive clone (Screening PCR) of the master plate was used to inoculate an over night culture of $100 \mathrm{ml}$ (LB medium $+1 \%$ glucose supplemented with $100 \mu \mathrm{g} / \mathrm{ml}$ ampicillin). For long-term storage of the clones glycerol stocks of the over night culture (26\% glycerol) were prepared.

For DNA isolation, the cells were harvested by centrifugation (15 min, $5000 \mathrm{rpm}$ (J2-MC, rotor JA14), $4{ }^{\circ} \mathrm{C}$ ) and the plasmid DNA was isolated using the PureLink HiPure Plasmid Midiprep Kit (Invitrogen) according to the manufacturer's instruction. The DNA was air-dried, the pellet dissolved in $100 \mu \mathrm{dH}_{2} \mathrm{O}$ and stored at $-20{ }^{\circ} \mathrm{C}$. To confirm the identity of the resulting DNA a control digestion with appropriate restriction enzymes and/or sequence analysis was performed.

\subsubsection{Sequence Analysis}

Two $\mu \mathrm{g}$ of DNA was air dried and sent together with $30 \mu \mathrm{l}$ of primer (10 pmol/ $\mu \mathrm{l})$ to MWG biotech (Martinsried, $G$ ). Sequence alignment and analysis was performed using the program "blast" (Tatusova \& Maddrn, 1999) and the program Clone Manager 7 respectively.

\subsubsection{Photometric Measurement of DNA concentration}

DNA absorbance was measured photometrically at $\mathrm{OD}_{260}$ and the concentration calculated 
by the formula:

$$
c_{D N A}[\mu g / \mu /]=O D_{260} * \text { dilution factor } * 0.05
$$

\subsubsection{Expression and Purification of scFv' variants}

\subsubsection{Periplasmic protein expression in E. coli}

All scFv'36 constructs were produced in the periplasma of E. coli TG1. For a $2 \times 1$ I production, $25 \mathrm{ml}$ of over night culture (2x TY medium supplemented with $1 \%$ glucose, 100 $\mu \mathrm{g} / \mathrm{ml}$ of ampicillin) were inoculated from a glycerol stock and grown at $37^{\circ} \mathrm{C}$. The next day, $10 \mathrm{ml}$ of the over night culture were used to inoculate 1 I medium ( $2 x$ TY with $0.1 \%$ glucose, $100 \mu \mathrm{g} / \mathrm{ml}$ ampicillin) and cells were grown at $37{ }^{\circ} \mathrm{C}$ to $\mathrm{OD}_{600}$ of $0.8-1.0$ in a $2 \mathrm{~L}$ baffled shaking flask on a rotary shaker at 180-190 rpm. Induction of the antibody under lactose promoter was started by adding IPTG (f.c. $0.1 \mathrm{M}$ ), followed by incubation at room temperature for $3 \mathrm{~h}$ whilst shaking (180-190 rpm). The cells were harvested (10 min, 5000 rpm, J2-MC, rotor JA10) and resuspended in $50 \mathrm{ml}$ periplasmatic protein preparation buffer. Cell wall lysis was achieved by adding lysozyme (f.c. $50 \mu \mathrm{g} / \mathrm{ml}$ ) [Roche, Mannheim, G] and incubation for 20 minutes on ice. Spheroblasts were stabilized by addition of $\mathrm{MgCl}_{2}$ (f.c. 0.01 M). The supernatant obtained after centrifugation (10 min, $8000 \mathrm{rpm}$, rotor JA14) was dialyzed (MWCO 8-10 kDa) against 5 I PBS over night $\left(4^{\circ} \mathrm{C}\right)$. The dialyzed supernatant was centrifuged again (15 min, $8000 \mathrm{rpm}$, rotor $\mathrm{JA} 14,4^{\circ} \mathrm{C}$ ) and recombinant protein purified by Ni-NTA-IMAC as described in (2.2.2.2).

\subsubsection{Purification by Immobilized Metal Affinity Chromatography (IMAC)}

The antibody fragments were purified by IMAC. A column was filled with $1 \mathrm{ml}$ of Ni-NTAagarose beads and equilibrated with $10 \mathrm{ml}$ sterile PBS. The protein suspension after dialysis was loaded onto the column. Unbound proteins were washed away with about $40 \mathrm{ml}$ of IMAC wash buffer (1x IMAC Na-phosphate buffer with $30 \mathrm{mM}$ imidazole) until no protein could be further detected in the collected wash fractions. Therefor a qualitative Bradford assay was carried out by adding $10 \mu \mathrm{l}$ of sample to $100 \mu \mathrm{l}$ of $1 \mathrm{x}$ Bradford reagent. The presence of protein was detected by the change of color. Bound proteins were eluted with IMAC column buffer (1x IMAC Na-phosphate buffer with $250 \mathrm{mM}$ imidazole) in fractions of $1 \mathrm{ml}$. The presence of protein in the elution and column wash fractions was detected by Bradford assay as described above. The purity of the protein in the eluted fractions was analyzed by SDSPAGE in comparison to crude extract, flow through and wash fraction. The fractions were pooled according to similar protein content (main fraction, side fractions) and dialyzed against $5 \mathrm{I}$ autoclaved PBS over night at $4{ }^{\circ} \mathrm{C}$ (MWCO $\left.12.4 \mathrm{kDa}\right)$. 


\subsubsection{Protein Characterization}

\subsubsection{Determination of Protein Concentration}

Protein concentration (c) was determined photometrically by measuring the absorbance at $280 \mathrm{~nm}\left(\mathrm{OD}_{280}\right)$.

$$
\begin{aligned}
& c[\mathrm{mg} / \mathrm{m} /]=M[\mathrm{~mol} / \mathrm{l}] * M W[\mathrm{~g} / \mathrm{mol}]=\frac{O D_{280}}{\varepsilon} * M W \\
& \varepsilon=\left(\text { number }_{T r p} * 5540\right)+\left(\text { number }_{T y r} * 1480\right)
\end{aligned}
$$

$(\mathrm{M}=$ molarity, $\mathrm{MW}=$ molecular weight $[\mathrm{g} / \mathrm{mol}=\mathrm{Da}], \varepsilon=$ molar extinction coefficient $)$

\subsubsection{SDS-PAGE and Western Blot Analysis}

Purity and molecular mass of protein samples were analyzed by SDS-PAGE. Samples were mixed with $5 x$ reducing SDS loading buffer and boiled at $95{ }^{\circ} \mathrm{C}(5 \mathrm{~min})$. A prestained protein ladder was used for size determination. Gels were run at $50 \mathrm{~mA} / \mathrm{gel}$ for approximately 1 hour, stained with Coomassie solution for $1 \mathrm{~h}$ on a shaker and destained with Coomassie destain solution over night. Gels with different acrylamide concentrations according to the expected protein molecular mass were prepared:

\begin{tabular}{lcccc}
\hline & \multicolumn{3}{c}{ Running gel } & Stacking gel \\
& $\mathbf{1 0} \%$ & $\mathbf{1 2} \%$ & $\mathbf{1 5} \%$ & $\mathbf{5} \%$ \\
\hline $\mathrm{H}_{2} \mathrm{O}$ & $2.95 \mathrm{ml}$ & $2.45 \mathrm{ml}$ & $1.7 \mathrm{ml}$ & $2.1 \mathrm{ml}$ \\
$30 \%$ Acrylamide Mix & $2.5 \mathrm{ml}$ & $3 \mathrm{ml}$ & $3.75 \mathrm{ml}$ & $0.5 \mathrm{ml}$ \\
$1.5 \mathrm{M}$ Tris, $\mathrm{pH} 8.8$ & $1.9 \mathrm{ml}$ & $1.9 \mathrm{ml}$ & $1.9 \mathrm{ml}$ & - \\
$1.0 \mathrm{M}$ Tris, $\mathrm{pH} 6.8$ & - & - & - & $0.38 \mathrm{ml}$ \\
$10 \%$ SDS & $75 \mu \mathrm{l}$ & $75 \mu \mathrm{l}$ & $75 \mu \mathrm{l}$ & $30 \mu \mathrm{l}$ \\
$10 \%$ APS & $75 \mu \mathrm{l}$ & $75 \mu \mathrm{l}$ & $75 \mu \mathrm{l}$ & $30 \mu \mathrm{l}$ \\
TEMED & $3 \mu \mathrm{l}$ & $3 \mu \mathrm{l}$ & $3 \mu \mathrm{l}$ & $3 \mu \mathrm{l}$
\end{tabular}

\subsubsection{Western Blot Analysis}

The identity of a protein separated by SDS-PAGE was confirmed by Western Blot. The protein on the polyacrylamide gel was transferred by blotting for $1 \mathrm{~h}$ at $12 \mathrm{~V}$ onto a nitrocellulose membrane (semidry blot) and remaining binding sites blocked with $5 \%$ skim milk powder in PBS $+0.1 \%$ Tween20 for $1 \mathrm{~h}$ at room temperature on a shaker. For detection of recombinant proteins with $\mathrm{His}_{6}$-tag the membrane was then incubated with an HRP conjugated anti-His ${ }_{6}$-tag antibody, diluted 1:1000 in blocking solution for $1 \mathrm{~h}$ at room temperature. After washing three times with PBS $+0.05 \%$ Tween20 and once with PBS alone for 5 minutes each, the blot was developed with ECL substrate solution ( $5 \mathrm{ml}$ Solution A $+500 \mu \mathrm{l}$ Solution $\left.\mathrm{B}+1.5 \mu \mathrm{H}_{2} \mathrm{O}_{2}(30 \%)\right)$ for 2 minutes in the dark. The blot was exposed 
to a light-sensitive film for 10 seconds to 1 minute and developed in a film developing machine.

\subsubsection{Size Exclusion Chromatography by High Performance Liquid Chromatography}

Analytical gel filtration was performed to determine the molecular mass and oligomerization state of the recombinant antibody samples under native conditions. $25 \mu \mathrm{l}$ of a sample with a concentration of $0.4-0.5 \mathrm{mg} / \mathrm{ml}$ was applied to a HPLC column (BioSep-SEC-S2000) at a flow rate of $0.5 \mathrm{ml} / \mathrm{min}$. For determining the size of recombinant proteins, standard proteins of size between $6.5 \mathrm{kDa}$ - $669 \mathrm{kDa}$ (aprotinin-6.5 kDa, cytochrome c-12.4 kDa, carbonic anhydrase-29 kDa, BSA-66 kDa, $\beta$-amylase-200 kDa, apoferritin-443 kDa, thyroglobulin$669 \mathrm{kDa}$ ) were run under the same conditions. Sample protein sizes were obtained by interpolation of a standard curve (retention times vs. protein sizes) in GraphPad Prism v4.0 (two phase exponential decay).

\subsubsection{Determination of Protein Melting Points}

The melting point of the scFv' variants were determined with the ZetaSizer Nano ZS (Malvern). Approximately $150 \mu \mathrm{g}$ of purified scFv' protein was diluted in PBS to a total volume of $1 \mathrm{ml}$ and sterile filtered into a quartz cuvette. Dynamic laser light scattering intensity was measured while the temperature was increased in $1{ }^{\circ} \mathrm{C}$ intervals from 30 to 70 ${ }^{\circ} \mathrm{C}$ using 2 min equilibration time between each temperature step. The melting point was defined as the temperature at which the light scattering intensity dramatically increased.

\subsubsection{Liposome Technology}

\subsubsection{Liposome Preparation}

All liposomes were prepared by the film hydration-extrusion method and were composed of egg phosphatidylcholine (EPC), cholesterol and $\mathrm{mPEG}_{2000}-\mathrm{DSPE}$ at a molar ratio of 6.5:3:0.5. In addition all liposomes contained $0.3 \mathrm{~mol} \%$ Dil or DiO, respectively as fluorescent dye. Lipids and Dil were dissolved in chloroform, and a thin lipid film was formed in a round bottom flask by removing the solvents in a rotary evaporator at $42^{\circ} \mathrm{C}$ and subsequent drying under vacuum for at least $1 \mathrm{~h}$ at room temperature. The lipid film was hydrated with $1 \mathrm{ml}$ of $10 \mathrm{mM}$ HEPES buffer, pH 7.4 and vortexed until all components were dissolved. The final lipid concentration was $10 \mu \mathrm{mol}$ per $\mathrm{ml}$ of buffer. The resulting multilamellar vesicle dispersion was extruded 21 times through a $50 \mathrm{~nm}$ polycarbonate filter membrane using a LiposoFast extruder (Avestin, Ottawa) to obtain small unilamellar vesicles. 


\subsubsection{Coupling scFv' to Micelles}

For the preparation of Mal-PEG ${ }_{2000}$-DSPE micelles, chloroform was removed by incubation at room temperature. The lipid was dissolved in $\mathrm{ddH}_{2} \mathrm{O}$ to a final concentration of $10 \mathrm{mg} / \mathrm{ml}$ and incubated in $5 \mathrm{~min}$ at $65^{\circ} \mathrm{C}$ in a water bath by shaking from time to time for the formation of micelles. For coupling scFv' molecules to maleimide, purified scFv' was reduced by adding 1

$\mu \mathrm{l}$ TCEP per $20 \mu \mathrm{g}$ protein and incubating under nitrogen atmosphere at room temperature for 2 to 3 hours. TCEP was removed by dialysis (Dialyzer Mini MWCO 6-8 kDa) against deoxygenated (30 min) coupling buffer ( $\mathrm{pH} \mathrm{6.7)} \mathrm{o/n} \mathrm{under} \mathrm{constant} \mathrm{stirring} \mathrm{at} 4^{\circ} \mathrm{C}$.

Micellar lipid and scFv' were mixed in a molar ratio of 4.67:1 (Nellis et al., 2005) and overlayed with nitrogen. The coupling reaction was performed at room temperature for 30 min and quenched with $1 \mathrm{mM} \mathrm{L-cysteine} \mathrm{for} \mathrm{at} \mathrm{least} 10 \mathrm{~min}$.

\subsubsection{Analysis of Coupling Efficiency}

The scFv'-coupled micelles and purified scFv' $(2 \mu \mathrm{g})$ were analyzed by $15 \%$ SDS-PAGE under reducing conditions and stained with Coomassie (2.2.3.2). The coupling efficiency was determined by quantifying the intensity of the protein bands before and after coupling to micelles using the software ImageQuant (GE Healthcare).

\subsubsection{Generation of Immunoliposomes}

The scFv'-coupled Mal-PEG 2000 -DSPE micelles were inserted into preformed PEGylated liposomes by incubation at $55{ }^{\circ} \mathrm{C}$ for $30 \mathrm{~min}$ with molar ratios of $0.6,2$ or $5 \mathrm{~mol} \%$, respectively, micellar lipid in respect to liposomal lipid. Non-coupled single-chain fragment was removed from immunoliposome preparations by gel filtration using a Sepharose CL4B column equilibrated with $10 \mathrm{mM}$ HEPES buffer, $\mathrm{pH}$ 7.4. Pink (Dil) fractions, indicating liposome-containing fractions were pooled.

\subsubsection{Determination of Liposome Size and $\zeta$-potential}

The liposomal formulations were diluted 1:50 in sterile filtered PBS. Size and zeta potential were measured using the Zetasizer Nano ZS.

For determination of the lipid concentration the initial amount of lipid loaded onto the column was divided by the final volume after gel filtration, not taking into account that part of the liposomes might have been lost during the gel filtration step. 


\subsubsection{Lipid coating of Nanoparticles}

\subsubsection{Lipid Coating by Extrusion}

A lipid film composed of EPC/Chol/mPEG ${ }_{2000}$-DSPE $=6.5: 3: 0.5$ or 6.8:3:0.2) without Dil was generated as described above (2.2.4.1). $200 \mu \mathrm{g}$ of polystyrene particles were added to 300 $\mu \mathrm{l}$ of hydrated lipid film and incubated for $30 \mathrm{~min}$ at $60{ }^{\circ} \mathrm{C}$, followed by extrusion through a $200 \mathrm{~nm}$ polycarbonate membrane. The resulting mixture of uncoated particles, lipid-coated particles (LP) and empty liposomes was added on the top of a sucrose gradient (6-15\% sucrose in water $(\mathrm{w} / \mathrm{w}))$ in a $10 \mathrm{ml}$ centrifuge tube. The density centrifugation was performed for $20 \mathrm{~h}$ at $68000 \mathrm{~g}$ (L7 ultracentrifuge, rotor SW41 Ti, Beckmann, G) (4 $\left.{ }^{\circ} \mathrm{C}\right)$. The LP fraction was carefully removed from the sucrose gradient and dialyzed against 5 I 10 mM HEPES buffer pH 7.4 over night at $4{ }^{\circ} \mathrm{C}$ (MWCO $\left.12.4 \mathrm{kDa}\right)$. The dialyzed lipid-coated particles were concentrated by centrifugation for $20 \mathrm{~min}$ at $16000 \mathrm{~g}$ and resuspension of the pellet in $200 \mu \mathrm{l}$ $10 \mathrm{mM}$ HEPES buffer and a washing step was carried out (20 min, 16 000g). The resulting pellet was resuspended in 100-200 $\mu \mathrm{l} 10 \mathrm{mM}$ HEPES buffer $\mathrm{pH} 7.4$.

\subsubsection{Lipid Coating by Sonification}

Liposomes composed of $\mathrm{EPC} / \mathrm{Chol} / \mathrm{mPEG}_{2000}-\mathrm{DSPE}=6.5: 3: 0.5$ or $\left.6.8: 3: 0.2\right)$ without $\mathrm{Dil}$ were generated as described above (2.2.4.1). The polystyrene nanoparticles (200 $\mu \mathrm{g})$ were added to $300 \mu$ l of preformed liposomes $(100 \mathrm{~nm})$ and sonificated in a water bath for $20 \mathrm{~min}$ at $60{ }^{\circ} \mathrm{C}$. LP were separated from empty liposomes by centrifugation for $20 \mathrm{~min}$ at $16000 \mathrm{~g}$. After an additional washing step the LP were resuspended in 100-200 $\mu 10$ mM HEPES buffer $\mathrm{pH} 7.4$.

\subsubsection{Generation of Targeted Lipid-Coated Nanoparticles}

Anti-FAP targeted lipid-coated particles (TLP) were generated by postinsertion of scFv36 LCH3-conjugated micelles into the lipid coat of LPs. The scFv'36 LCH3 Mal-PEG ${ }_{2000}-\mathrm{DSPE}$ micelles were prepared as describe in 2.2.4.2. The scFv-coupled micelles were inserted into the LP by incubation for $30 \mathrm{~min}$ at $55^{\circ} \mathrm{C}$ with molar ratios of $0.2,0.6,2$ and $5 \mathrm{~mol} \%$ micellar lipids in respect to LP lipid. Unbound scFv'36 LCH3 molecules and not inserted scFvmicelles were removed by centrifugation for $10 \mathrm{~min}$ at $68000 \mathrm{~g}$ (ultracentrifuge Optima TL). TLP were resuspended in $200 \mu \mathrm{l} 10 \mathrm{mM}$ HEPES buffer $\mathrm{pH}$ 7.4. Size of LP and TLP were measured using a ZetaSizer Nano ZS (2.2.4.5). Particle concentration of LP and TLP were determined by absorbance at 300-350 nm (Tecan infinite M200). Therefor, a standard curve of non coated polystyrene particles was measured in a range of 2 to $20 \mu \mathrm{g}$ P per $100 \mu \mathrm{l}$ PBS. Two and $4 \mu \mathrm{l}$ of the particle preparations were added to $100 \mu \mathrm{l}$ PBS and the absorbance was 
measured (300-350 nm). Obtained data were analyzed with GraphPad Prism 5 and the concentrations of LP and TLP were determined by linear regression.

\subsubsection{Binding Studies}

\subsubsection{Cell culture}

To start cultivation, frozen cell suspension (2 to 4 mio. cells in $1 \mathrm{ml} 10 \%$ DMSO in FCS) was quickly thawed in a $37^{\circ} \mathrm{C}$ water bath and mixed with $1 \mathrm{ml}$ of culture medium. The cells were centrifuged at $1500 \mathrm{rpm}$ (Eppendorf 5810R) for $5 \mathrm{~min}$ at RT, dissolved in fresh culture medium and transferred to culture flasks. Cells were passaged every two to three days. Adherent cells were detached using working solution of Trypsin/EDTA. Trypsin activity was then quenched by addition of medium. Cells were diluted 1:10 to 1:20 with fresh medium for further cultivation. $\mathrm{HT} 1080 \mathrm{FAP}_{\text {mo }}$ and $\mathrm{HT} 1080 \mathrm{FAP}_{\text {hu }}$ cultures were supplemented with $\mathrm{G} 418$ (f. c. $250 \mu \mathrm{g} / \mathrm{ml}$ ).

In order to store cells approximately $5 \times 10^{6}$ cells were resuspended in $1 \mathrm{ml} \mathrm{FCS,} 10 \%$ DMSO and gradually frozen to $-80^{\circ} \mathrm{C}$ in a cryobox filled with isopropanol. Cells were stored at $-80^{\circ} \mathrm{C}$ or in liquid nitrogen.

\subsubsection{Flow Cytometry}

To determine binding of different scF'36 variants to target antigen-expressing cells,

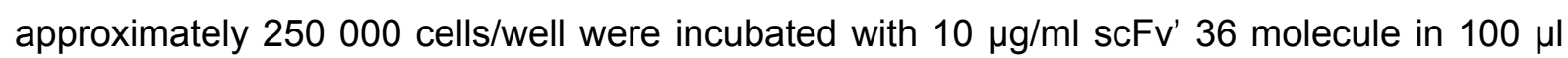
PBA per well in a v-shape 96-well cell culture plate for $1 \mathrm{~h}$ at room temperature. The cells were washed three times with $100 \mu \mathrm{l}$ PBA (5 min, $1500 \mathrm{rpm}$, Eppendorf 5810R) and incubated with FITC-conjugated anti-His-tag antibody diluted 1/500. After further washing steps (2 times), the cells were resuspended in $500 \mu \mathrm{l}$ PBA, transferred into FACS tubes and analyzed in a flow cytometer (EPICS or FC 500). In case of analyzing Dil-labeled immunoliposomes and particle preparations, cells were seeded as described above and incubated with $10 \mathrm{nmol}$ liposomal lipids or $3 \mu \mathrm{g}$ particles in $100 \mu \mathrm{l}$ PBA for $1 \mathrm{~h}$ at $4{ }^{\circ} \mathrm{C}$. Particles were detected by the incorporated green-fluorescing dye perylene monoimide (PMI). Washing steps and analysis was carried out as described above. For the competition experiments, liposomes and particle preparations were mixed with $10 \mu \mathrm{g} / \mathrm{ml}$ scFv36 prior to incubation with cells. Obtained data were analyzed with the software WinMDI, version 2.9.

\subsubsection{In Vitro Plasma Stability}

To analyze the stability under physiological conditions, anti-FAP immunoliposomes (10 nmol) or anti-FAP TLP (corresponding to $3 \mu \mathrm{g}$ particles) were preincubated in the presence of PBS 
or $50 \%$ human plasma (stabilized with citrate-phosphate-dextrose solution (CPD)) for up to 4 days or $24 \mathrm{~h}$, respectively, in a total volume of $50 \mu \mathrm{l}$. Thereafter, binding to FAP-positive and FAP-negative cells were determined by flow cytometry as described above (2.2.7.2).

\subsubsection{Internalization Studies by Microscopy}

\section{Anti-FAP immunoliposomes}

Autoclaved cover slips were coated with collagen R (f. c. $25 \mu \mathrm{g} / \mathrm{ml}$ ) for $2 \mathrm{~h}$ at $37{ }^{\circ} \mathrm{C}$ and rinsed twice with $1 \mathrm{ml}$ PBS. Subsequently, Ht1080 wild-type and $\mathrm{FAP}_{\text {hu }}$ cells $\left(0.5 \times 10^{5}\right.$ cells $/ \mathrm{ml}$ ) were seeded onto coated cover slips and cultivated over night at $37{ }^{\circ} \mathrm{C}$ and $5 \%$ $\mathrm{CO}_{2}$ in $1 \mathrm{ml}$ culture medium. Next day culture medium was replaced with $1 \mathrm{ml}$ fresh medium and adherent cells were incubated with DiO-labeled liposomes $(20 \mathrm{nmol})$ for up to 6 hours at $37^{\circ} \mathrm{C}$. Afterwards the cells were washed twice with $1 \mathrm{ml}$ ice-cold PBS and fixed with $4 \%$ para-formaldehyde (PFA) for $20 \mathrm{~min}$ at room temperature. Again the cells were washed twice in ice-cold PBS. The nuclei were stained in DAPI solution (f. c. $1 \mu \mathrm{g} / \mathrm{ml}$ ) for $20 \mathrm{~min}$ at room temperature in the dark. Prior mounting the cover slip upside down in a drop of Mowiol, they were washed with PBS. Slides were analyzed by a fluorescent microscope (Cellobserver). Excitation at $488 \mathrm{~nm}$ induces the DiO fluorescence (green emission) for the immunoliposomes, while excitation at $364 \mathrm{~nm}$ induces DAPI fluorescence (blue emission). The cell outlines are visualized in bright field settings.

\section{Anti-FAP targeted-lipid-coated nanoparticles}

Antigen-expressing and control cells $\left(0.2 \times 10^{5}\right.$ cells $\left./ \mathrm{ml}\right)$ were incubated in $1 \mathrm{ml}$ culture medium with $10 \mu \mathrm{g}$ of particles, LP and TLP, respectively for $6 \mathrm{~h}$ at $37^{\circ} \mathrm{C}$ under permanent rolling. To separate the particle preparation from the cells, they were underlayed with $1 \mathrm{ml}$ FCS and centrifuged for $5 \mathrm{~min}$ at $300 \mathrm{~g}$ at $4^{\circ} \mathrm{C}$ (Eppendorf $415 \mathrm{R}$ ). The supernatant was carefully removed and cells were washed twice with $200 \mu$ ice-cold PBS and fixed in $800 \mu \mathrm{l}$ of $4 \%$ para-formaldehyde (PFA) for $20 \mathrm{~min}$ at room temperature. Afterwards the cells were washed twice in ice-cold PBS and resuspended in $20 \mu \mathrm{l}$ Mowiol. Cells were analyzed by fluorescent microscopy (Cellobserver).

In case of visualizing cell boundary, the cells were additionally incubated in $1.4 \mu \mathrm{l}$ Alexa Fluor ${ }^{\circledR} 488$ anti-human CD105 antibody in $200 \mu$ PBA for 30 min on ice to stain membranebound endoglin. Afterwards the cells were washed twice with $200 \mu \mathrm{l}$ ice-cold PBS and fixed with $800 \mu \mathrm{l}$ of $4 \%$ para-formaldehyde (PFA) for $20 \mathrm{~min}$ at room temperature. Again the cells were washed twice in ice-cold PBS and resuspended in $20 \mu \mathrm{l}$ Mowiol. Cells were analyzed by confocal microscopy (Leica TCS SP 2). 


\subsubsection{Pharmacokinetic}

Animal care and all experiments performed were in accordance with federal guidelines and have been approved authorities of the university and of the state Baden-Wuerttemberg. Amino-functionalized particles containing an IR-fluorescent dye were used for the generation of lipid-coated particles. CD1 mice (female, 11-17 weeks, weight between 32-40 g, 6 mice/group) received an i.v. injection of $300 \mu \mathrm{g}$ particle or LP in a total volume of $300 \mu \mathrm{l}$ PBS. In time intervals of $3,10,30,60,120,240 \mathrm{~min}$ and $24 \mathrm{~h}$ blood samples (100 $\mu \mathrm{l})$ were taken from the tail and incubated for at least $1 \mathrm{~h}$ on ice. Clotted blood was centrifuged at $300 \mathrm{~g}$ for $60 \mathrm{~min}$ and serum samples stored at $-20^{\circ} \mathrm{C}$. Serum concentrations of particle or LP were determined by LI-COR Odyssey [LI-COR bioscience]. Therefor, $15 \mu \mathrm{l}$ of selected serum was mixed with $85 \mu \mathrm{I}$ PBS and analyzed by LI-COR Odyssey by means of inserted IR-fluorescent dye (700 nm channel). Concentrations were determined by linear regression of a standard curve of uncoated particles. Initial injected dose was set to $100 \%$. Pharmacokinetic parameters AUC, $t_{1 / 2} \alpha$ and $t_{1 / 2} \beta$ were calculated with Excel using the first 3 times points to calculate $t_{1 / 2} \alpha$ and the last 3 time points to calculate $t_{1 / 2} \beta$.

\subsubsection{Coupling of Cys-scTNF to Nanoparticles}

Cys-scTNF was produced by Appronex (Prague, Czech Republic) and stored in $20 \mathrm{mM}$ HEPES, $150 \mathrm{mM} \mathrm{NaCl}, 1 \mathrm{mM}$ DTT, $0.1 \mathrm{mM} \mathrm{CaCl}_{2}, 1 \%$ sucrose, 0.01\% Tween-20, pH 7.4. DTT was removed by dialysis (Dialyzer Mini MWCO 6-8 kDa) for $4 \mathrm{~h}$ against oxygenated (30 min) coupling buffer ( $\mathrm{pH}$ 6.7). The solution was sterilized by filtration. One milligram of amino-functionalized polystyrene particles was suspended in $900 \mu \mathrm{l}$ of a $3 \mathrm{mM}$ sulfosuccinimidyl-4-(N-maleimidomethyl) cyclohexane-1-carboxylate (sulfo-SMCC) solution in PBS pH 7.4 and the reaction mixture was shaken for $1 \mathrm{~h}$ at room temperature. All steps were performed under sterile conditions. The resulting particles were collected by centrifugation for $10 \mathrm{~min}$ at $68000 \mathrm{~g}$ (ultracentrifuge Optima $\mathrm{TL}$ ) at $4{ }^{\circ} \mathrm{C}$ and washed with $500 \mu \mathrm{l}$ coupling buffer $\mathrm{pH}$ 6.7. Subsequently the activated particles were resuspended in a sterile solution of Cys-scTNF (1,10 or $30 \mu \mathrm{g})$ in coupling buffer and incubated for $1 \mathrm{~h}$ at RT under constant rolling. The reaction was quenched with $1 \mathrm{mM}$ L-cysteine, $0.02 \mathrm{mM}$ EDTA, $\mathrm{pH}$ 5.5. Uncoupled Cys-scTNF was removed by centrifugation for $10 \mathrm{~min}$ at $68000 \mathrm{~g}$ (ultracentrifuge Optima TL) at $4{ }^{\circ} \mathrm{C}$. Afterwards, the particles were washed twice (centrifugation step between the washings) with sterile PBS. The final preparation was resuspended in $200 \mu \mathrm{l} \mathrm{PBS}$ and stored at $4{ }^{\circ} \mathrm{C}$. Bioactivity of the particles was determined in cytotoxicity assays as described below. Coupling efficiency was determined by sandwich ELISA (Hycult biotechnology). Firstly, the amount of Cys-scTNF in the supernatant incubated 
without $\mathrm{P}$ was determined (= Cys-scTNF input). Secondly, the amounts of free Cys-scTNF in the supernatant after coupling to $P$ and after two washing steps were determined, added together and subtracted from the Cys-scTNF input yielding the amount of Cys-scTNF coupled to $1 \mathrm{mg}$ of $P$.

\subsubsection{Cytotoxicity Assays}

Kym-1 cells $\left(1.5 \times 10^{4}\right.$ cells/well) were grown in 96-well flat bottom cell culture plates in 100 $\mu \mathrm{l}$ cell culture medium over night. TNF derivatives were diluted in culture medium and $50 \mu \mathrm{l}$ were added to each well, with final TNF concentrations ranging from 0 to $10 \mathrm{ng} / \mathrm{ml}$. In case of scTNF-particle preparations, dilutions in the range of 0 to $66.7 \mu \mathrm{g} \mathrm{P} / \mathrm{ml}$ were added and cells were cultivated over night. The next day, supernatant was discarded. Viable cells were stained in $50 \mu \mathrm{l}$ crystal violet solution ( $20 \%$ methanol, $0.5 \%$ crystal violet) for $15 \mathrm{~min}$ at room temperature. The wells were washed with $\mathrm{H}_{2} \mathrm{O}$ and air-dried. The dye was resolved with $50 \mu \mathrm{l}$ methanol for $15 \mathrm{~min}$, and optical density at $550 \mathrm{~nm}$ was determined (Tecan infinite M200). Data were analyzed using the software Microsoft ${ }^{\circledR}$ Excel and GraphPad Prism 5.

HT1080 wild-type or HT1080 FAP $_{\text {hu }}$ cells $\left(1.5 \times 10^{4}\right.$ cells $\left./ \mathrm{ml}\right)$ were incubated with different amounts (0-15 $\mu \mathrm{g}$ particles per $\mathrm{ml})$ of $\mathrm{P}, \mathrm{LP}$ and TLP, respectively, in medium containing cycloheximide ( $\mathrm{CHX}$ ) (f. c. $2.5 \mu \mathrm{g} / \mathrm{ml}$ ) for $2 \mathrm{~h}$ at $37^{\circ} \mathrm{C}$ under permanent rolling. To separate the cells from unbound particles, the probes were then underlayed with $1 \mathrm{ml} \mathrm{FCS}$ and centrifuged at $300 \mathrm{~g}$ for $5 \mathrm{~min}$. The separated cells were taken up in $300 \mu \mathrm{l}$ culture medium containing $\mathrm{CHX}$ (f. c. $2.5 \mu \mathrm{g} / \mathrm{ml}$ ) and split into $100 \mu \mathrm{l}$ fractions which were transferred into 96well flat bottom plates and grown for $16 \mathrm{~h}$ at $37{ }^{\circ} \mathrm{C}$ and $5 \% \mathrm{CO}_{2}$. Subsequently, the cells were stained as describes above. 


\section{Results}

\subsection{Novel Single-Chain Fv' Formats for the Generation of Immunoliposomes by Site-Directed Coupling}

Immunoliposomes generated by coupling of antibodies to the liposomal surface allow an active tissue targeting, e.g. through binding to tumor cell-specific receptors. Instead of whole antibodies, single-chain $\mathrm{Fv}$ fragments (scFv), which represent the smallest part of an antibody containing the entire antigen-binding site, find increasing usage as targeting moiety. The insertion of an additional cysteine residue permits site-directed coupling to reactive groups of lipids. In this work a comparative analysis of various scFv' variants with one or three additional cysteine residues introduced either at a C-terminal extension of varying length or at different positions in the linker peptide was performed, using a scFv fragment directed against fibroblast activation protein (FAP) as model antibody (Baum et al., 2007).

\subsubsection{Generation and Characterization of scFv' Variants}

Six different scFv' variants of anti-FAP scFv 36 were designed. The variants $\mathrm{HC} 2$ and $\mathrm{HC} 4$ contain one more additional cysteine residues at the $\mathrm{C}$-terminal and vary in the length of the C-terminal extension (Figure 3-1). Variant HC3 contains three additional cysteine residues at the C-terminal extension, which also includes a hexahistidyl-tag for purification. Furthermore, three different $\mathrm{scFv}$ ' variants containing an additional cysteine residue in the peptide linker at different positions were designed (LC variants). These molecules exhibit a Myc-tag and hexahistidyl-tag at the $\mathrm{C}$-terminus.

a)

$$
\begin{aligned}
& \text { ScFv' HC2 } \mathrm{V}_{\mathrm{H}} \text { - } \mathrm{V}_{\mathrm{L}} \text {-AHHHHHHGGSSGSGC }
\end{aligned}
$$

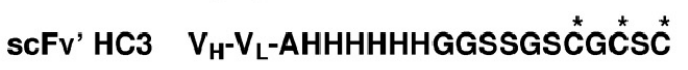

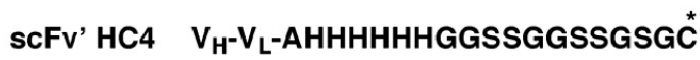

b)

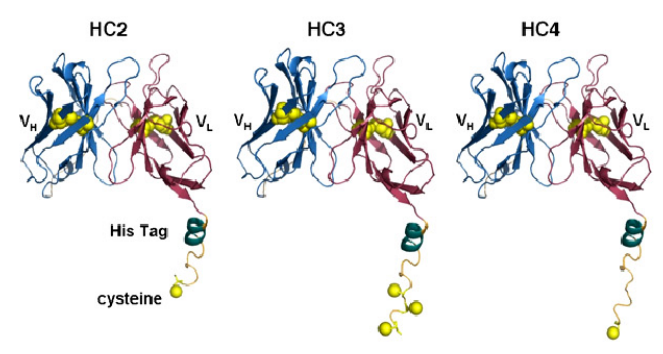

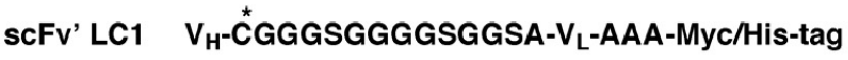

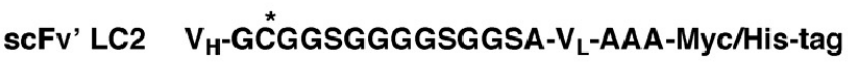

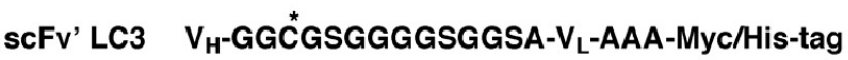

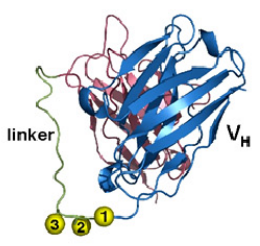

Figure 3-1: Composition of scFv' variants. Sulfur atoms of the cysteine residues are shown as spheres. (a) C-terminal sequences and model structure of $\mathrm{scFv}^{\prime} 36 \mathrm{HC}$ constructs (b) Linker sequences and model structure of the LC constructs. The Myc/His-tag has the sequence EQKLISEEDLNGAAHHHHHH-. Structures were visualized with PyMol (http://www.pymol.org). 
The model structures of each scFv'36 variant are presented in Figure 3-1. They were generated by Michael Knoll and Jürgen Pleiss (ITB, University Stuttgart). The models of the linker modifications ( $\mathrm{LC}$ and $\mathrm{LCH}$ ) indicate that the inserted cysteine residues are located at the bottom of the scFv' molecules opposite of the antigen binding site. Therefore the SHgroups should be accessible for coupling.

All scFv'36 variants could be expressed in E. coli TG1 and purified in soluble form from periplasmic preparations by IMAC. Protein yields varied between 0.2-0.8 mg per liter culture. For the HC variants, SDS-PAGE analysis showed a single band with an apparent molecular mass of $30 \mathrm{kDa}$ under reducing and nonreducing conditions (Figure 3-2 a, c). An additional band of $60 \mathrm{kDa}$ could be seen under nonreducing conditions (Figure 3-2 c), which corresponds to a dimer of two single-chain fragments. The LC constructs showed only a one prominent band of $32 \mathrm{kDa}$ under reducing and nonreducing conditions (Figure 3-2 a, c). Immunoblot experiments with anti-His-tag antibody confirmed the identity of the purified proteins (Figure 3-2 b, d). A second protein band of approximately $20 \mathrm{kDa}$ could be observed for the LC constructs only under reducing conditions. This is probably due to proteolytic cleavage.

a)

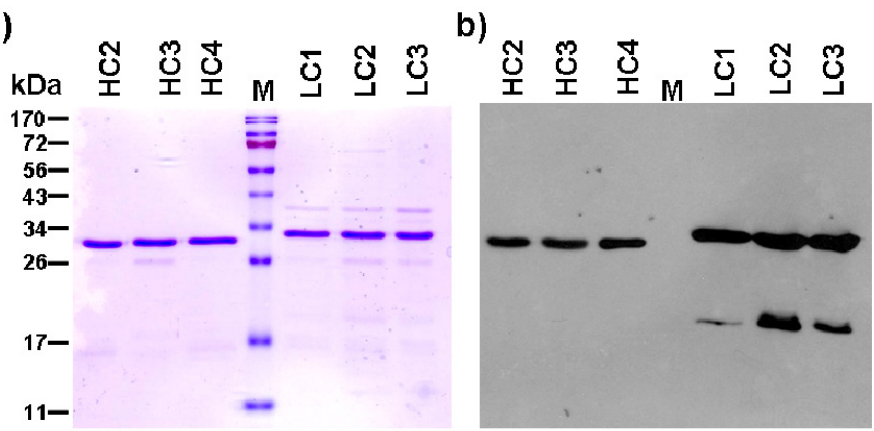

c)

d)

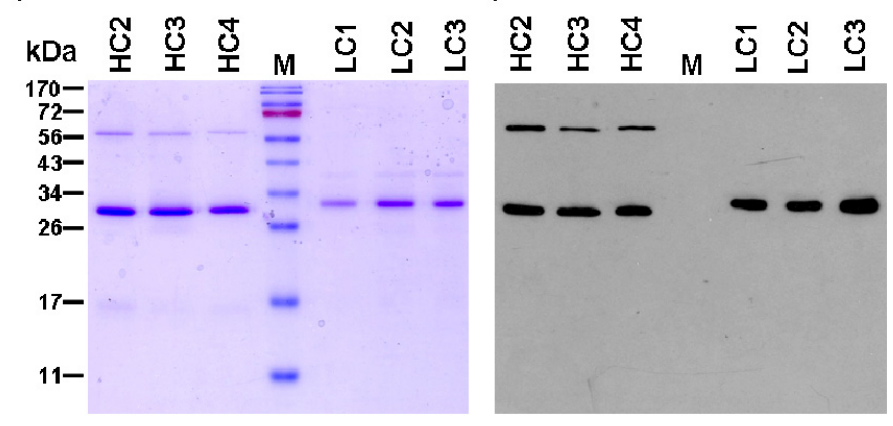

Figure 3-2: SDS-PAGE and immunoblot analysis of purified scFv' variants. Purified scFv' variants $\mathrm{HC} 2-4$ and LC1-3 were analyzed by $15 \%$ SDS-PAGE under nonreducing (c and d) or reducing $(a$ and $b)$ conditions and either stained with Coomassie or immunoblotted with an anti-His-tag antibody ( $2 \mu \mathrm{g} / \mathrm{lane}$ were used for Coomassie staining and $1 \mu \mathrm{g} / \mathrm{lane}$ for immunoblotting).

The scFv'36 variants showed a major peak in HPLC size exclusion chromatography (Figure 
3-3). The three $\mathrm{HC}$ constructs eluted with an apparent molecular mass of $22.5 \mathrm{kDa}$, which is somewhat below the calculated of $28 \mathrm{kDa}$. While the LC variants eluted with an apparent molecular mass of $29 \mathrm{kDa}$ (calculated mass, $28 \mathrm{kDa}$ ). A minor peak of higher molecular mass could be detected for the LC constructs representing the exclusion volume of the column. A small shoulder for the LC variants, in particular for LC2 indicating a dimer. A minor peak of lower molecular mass is presented for all constructs probably representing low molecular dirt in the samples that could not be detected by SDS-PAGE analysis.

$\mathrm{HC2}$

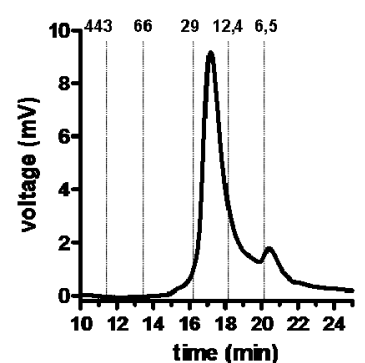

LC1

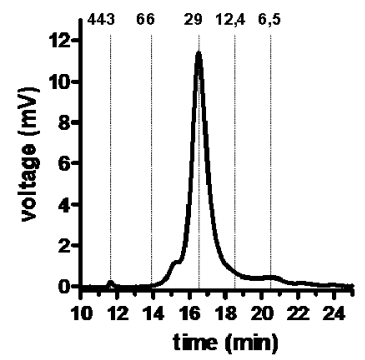

$\mathrm{HC} 3$

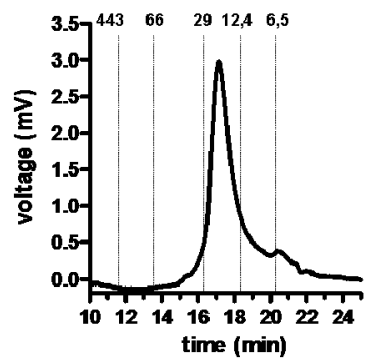

LC2

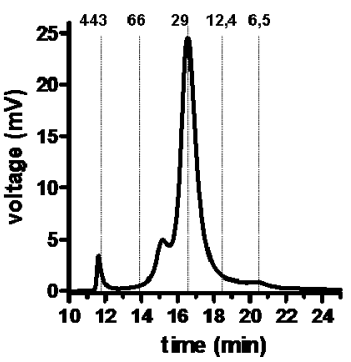

$\mathrm{HC} 4$

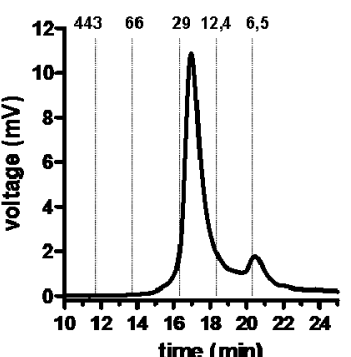

LC3

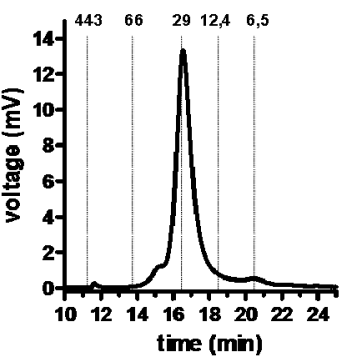

Figure 3-3: High-performance liquid chromatography. ScFv' molecules were analyzed by HPLC size exclusion chromatography using a BioSepSec-2000 column. Peak positions of standard proteins are indicated.

The thermal stability of each scFv'36 construct was determined by dynamic light scattering to investigate if these variants are suitable for the postinsertion method (Figure 3-4). For the HC constructs the melting point was determined to be around $56-57^{\circ} \mathrm{C}$. The LC constructs show a slightly higher melting point between $58-59^{\circ} \mathrm{C}$. 
HC2

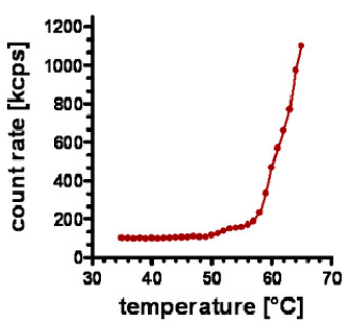

LC1

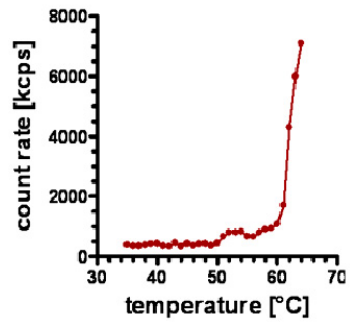

HC3

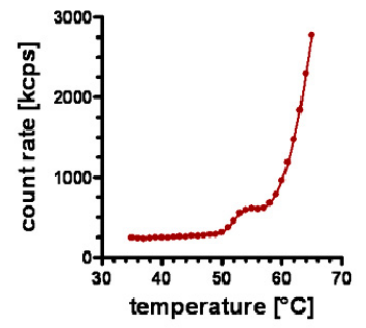

LC2

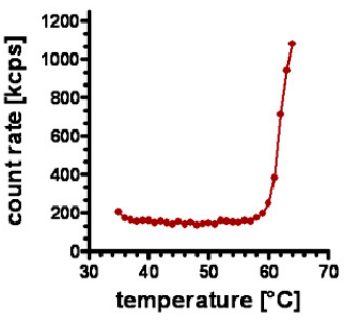

$\mathrm{HC4}$

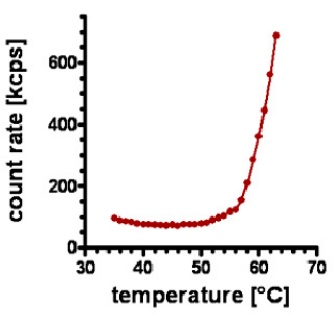

LC3

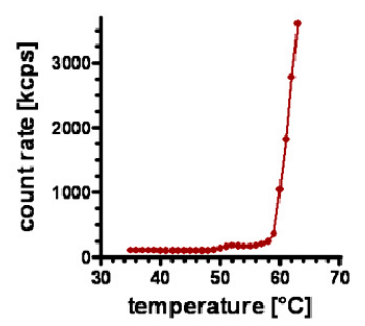

Figure 3-4: Thermal stability. Protein melting curves of the scFv' variants were determined by dynamic light scattering using ZetaSizer NanoZS $\left(1^{\circ} \mathrm{C}\right.$ intervals).

To show specific binding of the different scFv'36 constructs to target antigen-expressing cells flow cytometry was performed. Antigen-expressing cells (HT1080-FAP mo $_{\text {) }}$ and control cells (HT1080 wt) were incubated in PBA with added scFv' variant $(10 \mu \mathrm{g} / \mathrm{ml})$ or in PBA without scFv' as control. Bound scFv's were detected by FITC-conjugated anti-His-tag antibody. The results are summarized in Figure 3-5.
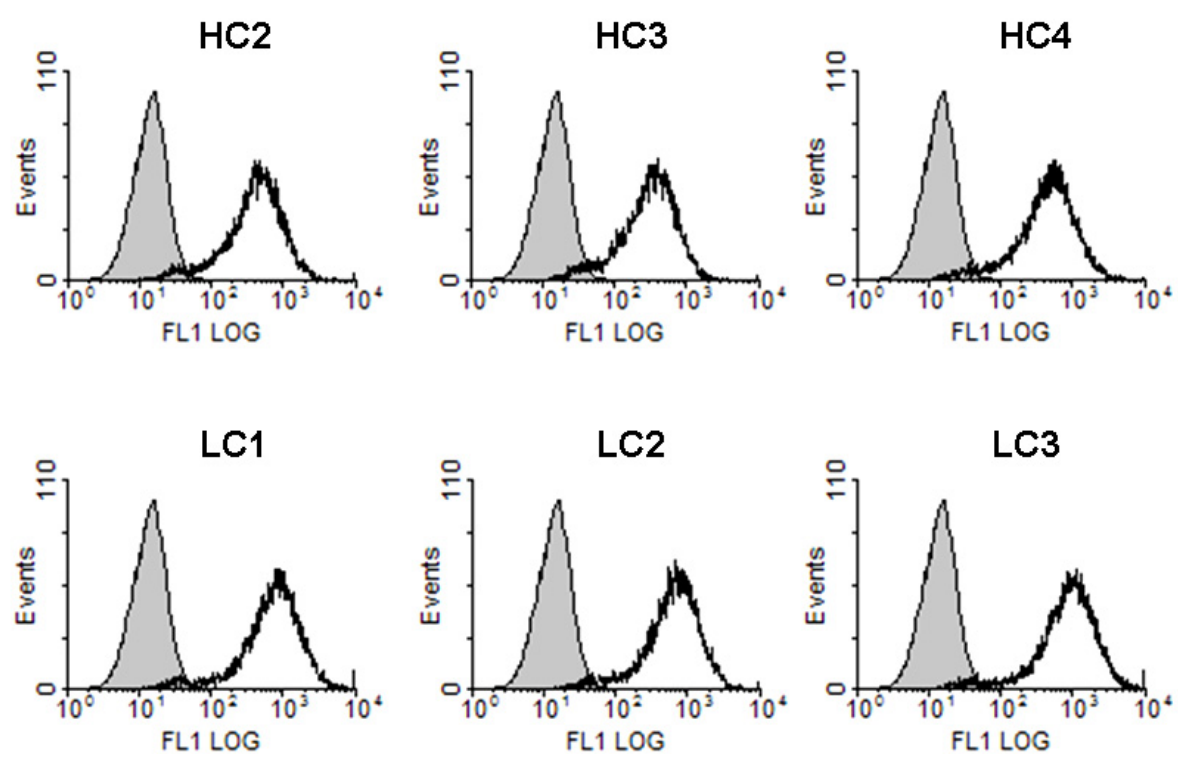

Figure 3-5: Binding analysis of scFv' variants to FAP-expressing cells by flow cytometry. ScFv' variants $(10 \mu \mathrm{g} / \mathrm{ml})$ were incubated with $250000 \mathrm{HT} 1080-\mathrm{FAP}_{\mathrm{mo}}$ cells. Grey fill: cells alone; black line: scFv' molecules $(10 \mu \mathrm{g} / \mathrm{ml}$, detection: FITC-conjugated anti-His-tag antibody $(1: 500))$. 
The HC and LC variants of scFv'36 showed binding to FAP-expressing cells (Figure 3-5) but not to wild-type cells (data not shown). This indicates that the additionally inserted cysteine residues at the $\mathrm{C}$-terminus or in the linker region do not interfere with the antigen binding activity.

\subsubsection{Coupling of scFv' Fragments to Mal-PEG $2000-D S P E$ Micelles}

The first step to generate immunoliposomes by the postinsertion method is the coupling of scFv' molecules to preformed Mal-PEG ${ }_{2000}$-DSPE-micelles. In this work a molar ratio of scFv' to lipid of 1:4.67 was used. The coupling reaction was performed at room temperature and was quenched after $30 \mathrm{~min}$. Successful coupling of the lipid to the scFv' fragments was shown by SDS-PAGE analysis (Figure 3-6). All scFv' variants possessing one additional cysteine residue showed an increase of the apparent molecular mass of approximately 3 $\mathrm{kDa}$. Only the molecular mass of the HC3 construct showed an increase of $10 \mathrm{kDa}$. These results indicate a coupling of one Mal-PEG ${ }_{2000}$-DSPE chain to one cysteine residue, possessing a molecular mass of $2.8 \mathrm{KDa}$.

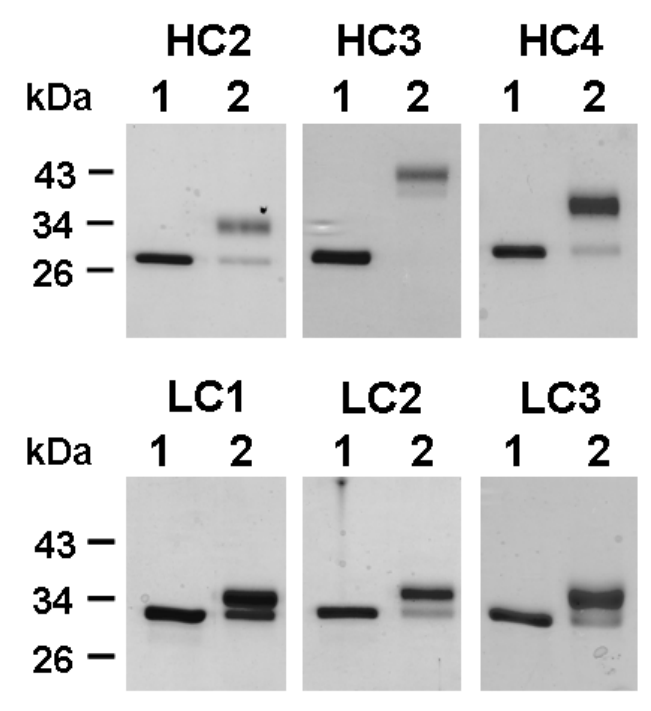

Figure 3-6: Coupling of ScFv' variants to Mal-PEG ${ }_{2000}$-DSPE micelles. SDS-PAGE analysis of scFv' fragments before (1) and after (2) coupling to micelles. Coupling of Mal-PEG-DSPE induce a mobility shift. Gel is stained with Coomassie.

The coupling efficiency of these reactions was determined by quantitative analysis of the scFv' molecules before and after the coupling to micelles. The results are presented in Table 2. The efficiencies were in the range of $80-98 \%$. The highest coupling efficiency was found for the HC3 variant with about $98 \%$. 
Table 2: Coupling efficiency of scFv' variants

\begin{tabular}{lc}
\hline construct & coupling efficiency (\%) \\
\hline ScFv'36 HC2 & $86.7 \pm 4.5$ \\
scFv'36 HC3 & $97.8 \pm 0.2$ \\
scFv'36 HC4 & $82.0 \pm 7.5$ \\
ScFv'36 LC1 & $79.7 \pm 4.7$ \\
scFv'36 LC2 & $81.8 \pm 3.9$ \\
scFv'36 LC3 & $90.2 \pm 2.3$
\end{tabular}

\subsubsection{Generation of scFv Immunoliposomes by the Postinsertion Method}

To generate scFv immunoliposomes, scFv-coupled micelles were inserted into preformed liposomes by incubation for $30 \mathrm{~min}$ at $55^{\circ} \mathrm{C}$ (Ishida et al., 1999; Allen at al., 2002). The liposomes were composed of egg phosphatidylcholine (EPC), cholesterol (Chol) and $\mathrm{mPEG}_{2000}$-DSPE at a molar ratio of 6.5:3:05. Two different micellar to liposomal lipid ratios (0.6 mol\% and $2 \mathrm{~mol} \%$ micellar lipid related to total lipid) were used to generate immunoliposomes. The resulting immunoliposomes possessed a size of 90-106 nm (Table 3). These average sizes are similar to the plain liposomes used for postinsertion of the micelles. The $\zeta$-potential of the immunoliposomes was determined to be slightly negative whereas the acceptor liposomes had a slightly positive $\zeta$-potential (Table 3). The scFv' density was calculated to be in the range of 1.1-1.2 nmol scFv/ $\mu \mathrm{mol}$ lipid at $0.6 \mathrm{~mol} \%$ micellar lipid and 3.7-3.9 nmol scFv/ $\mu \mathrm{mol}$ lipid at $2 \mathrm{~mol} \%$ micellar lipid.

Table 3: Size, Polydispersity Index (PDI) and ל-Potential of scFv Immunoliposomes Immunoliposomes were generated by insertion of scFv-coupled micelles into preformed liposomes (2 mol \% micellar lipids to total lipid).

\begin{tabular}{cccc}
\hline construct & size $(\mathrm{nm})$ & PDI & $\zeta$-potential $(\mathrm{mV})$ \\
\hline- & $108 \pm 2.3$ & $0.123 \pm 0.038$ & $5.2 \pm 3.5$ \\
scFv' 36-HC2 & $90 \pm 1.5$ & $0.159 \pm 0.058$ & $-12.6 \pm 5.4$ \\
scFv' 36-HC3 & $96 \pm 1.2$ & $0.134 \pm 0.032$ & $-12.5 \pm 2.8$ \\
scFv' 36-HC4 & $102 \pm 2.8$ & $0.156 \pm 0.034$ & $-15.0 \pm 0.5$ \\
ScFv' 36-LC1 & $106 \pm 3.7$ & $0.178 \pm 0.021$ & $-8.8 \pm 1.5$ \\
ScFv' 36-LC2 & $105 \pm 7.6$ & $0.149 \pm 0.004$ & $-11.2 \pm 7.9$ \\
scFv' 36-LC3 & $105 \pm 7.7$ & $0.112 \pm 0.054$ & $-2.8 \pm 2.3$
\end{tabular}

The immunoliposome preparations were analyzed for binding on FAP-expressing cells

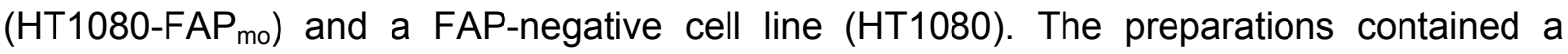
fluorescent dye incorporated into lipid layer for detection. 
scFv"

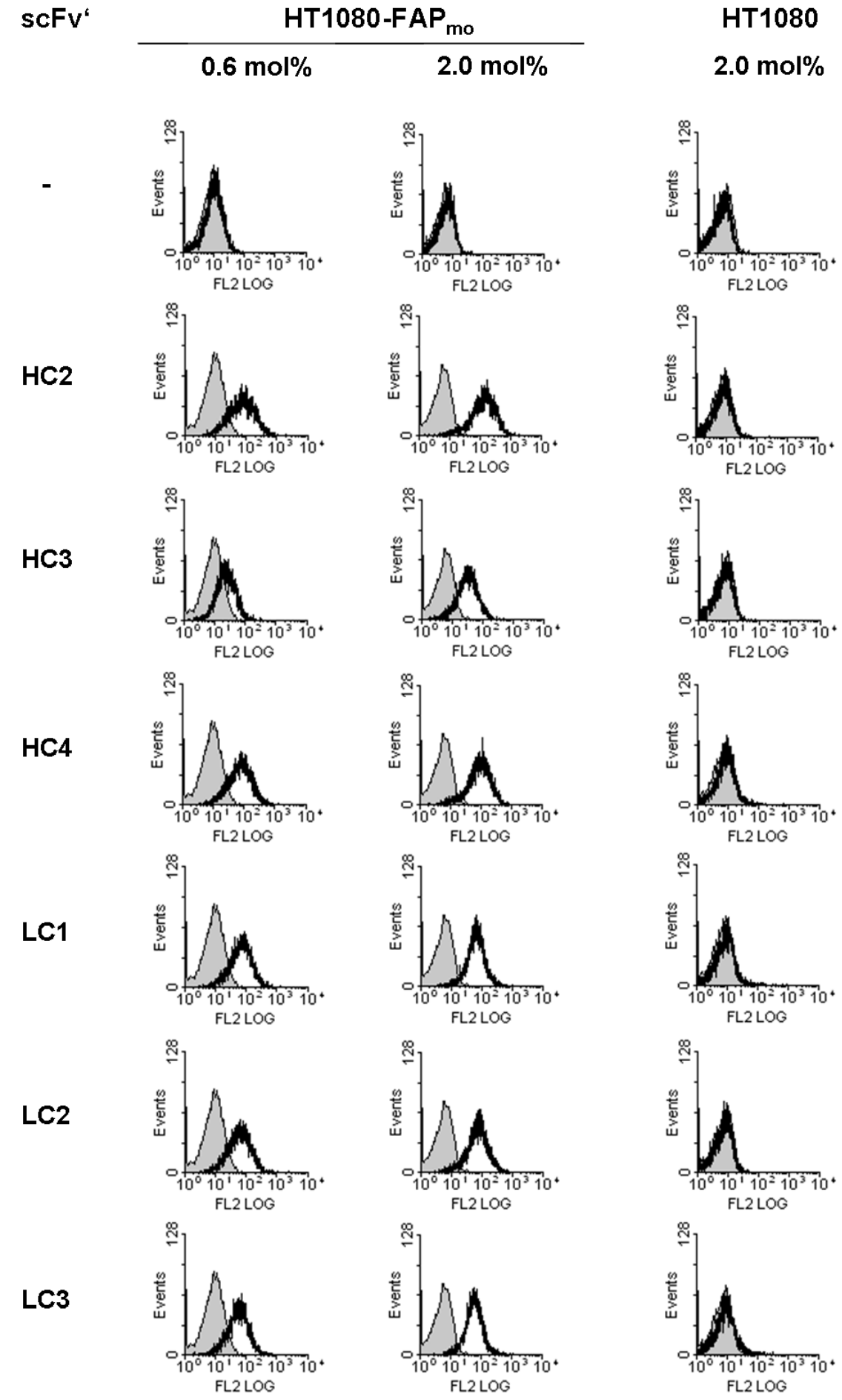

HT1080-FAP

HT1080

$2.0 \mathrm{~mol} \%$

Figure 3-7: Binding of scFv immunoliposomes to FAP-expressing cells. Micelle-coupled scFv's were inserted at two concentrations $(0.6 \mathrm{~mol} \%$ and $2 \mathrm{~mol} \%$ micellar lipids) into preformed PEGylated liposomes and analyzed for binding to FAP-expressing cells $\left(\mathrm{HT} 1080-\mathrm{FAP}_{\mathrm{mo}}\right)$ or to wild-type HT1080 cells. Negative control: plain liposomes (-), grey fill: cells alone, black line: cells incubated with liposomes at $10 \mathrm{nmol}$ lipid per 250.000 cells. 
All immunoliposomes showed a specific binding to $\mathrm{HT} 1080-\mathrm{FAP}_{\text {mo }}$ cells, whereas no binding was observed for wild-type cells (Figure 3-7). The plain liposomes without scFv showed no binding to both cell lines. The binding of the immunoliposomes was stronger for the higher micellar lipid concentration (2 mol\%) used by postinsertion. The immunoliposomes of different scFv-constructs showed a comparably strong binding, except HC3immunoliposomes, which showed a reduced binding under the applied assay conditions.

To verify that the scFv immunoliposomes bind only to FAP and not to any other molecule on the cell surface, binding was blocked by addition of soluble scFv' $36(10 \mu \mathrm{g} / \mathrm{ml})$. As a negative control the cells were also preincubated with an irrelevant ScFv (A5) directed against endoglin, which is also expressed on HT1080 cells (Rüger et al., 2006). The binding of the immunoliposomes was completely blocked by soluble scFv 36 for the $\mathrm{HC}$-immunoliposomes and almost completely for the LC-immunoliposomes, while no blocking was seen with scFv A5 for all immunoliposomes (Figure 3-8).

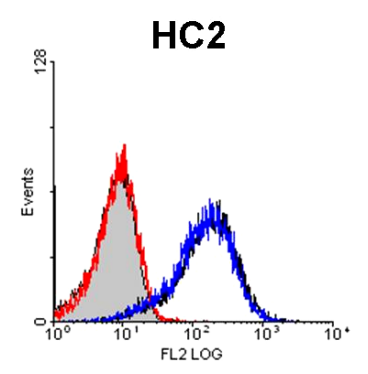

LC1

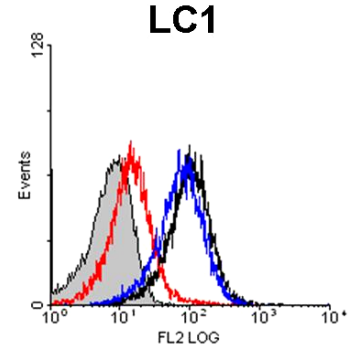

HC3

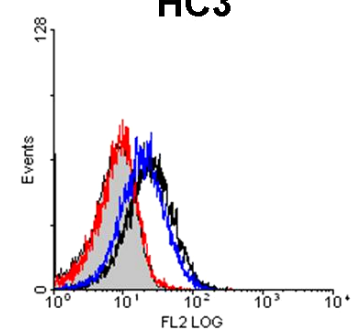

LC2

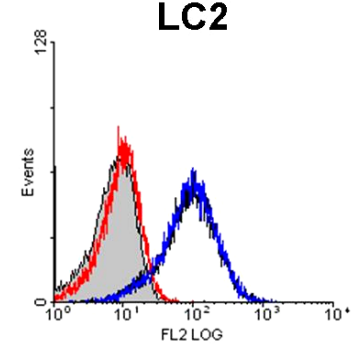

HC4

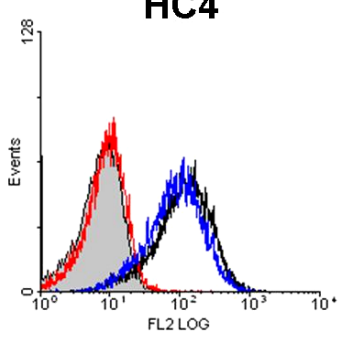

LC3

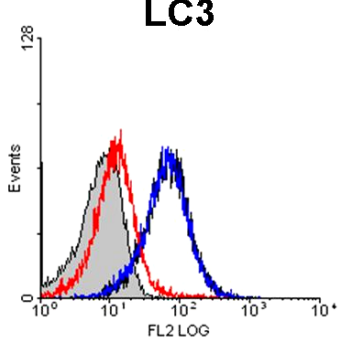

Figure 3-8: Blocking the binding of scFv immunoliposomes to HT1080 FAP mo $_{0}$ cells. Binding of immunoliposomes to HT1080 FAP mo $_{0}$ cells is blocked after preincubation with $10 \mu \mathrm{g}$ of soluble anti-FAP scFv 36 but not by an irrelevant scFv (A5). Grey fill: cells alone, black line: immunoliposomes $(10 \mathrm{nmol}$ lipid) alone, red line: immunoliposomes + scFv 36, blue line: immunoliposomes + scFv A5.

To analyze stability of these immunoliposomes under physiological conditions, immunoliposomes were incubated with human serum at $37^{\circ} \mathrm{C}$. No or only marginal reduction in binding to FAP-expressing cells after 4 days could be shown (Figure 3-9). 


\section{$\mathrm{HC2}$}

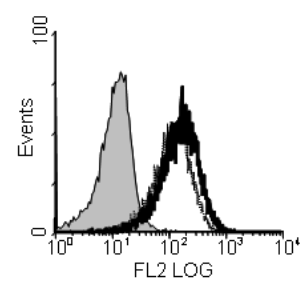

LC1

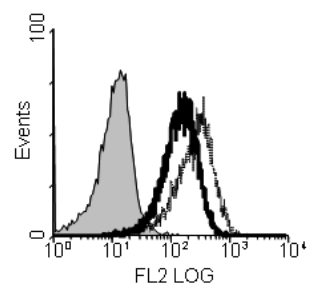

HC3

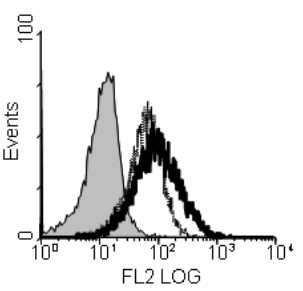

LC2

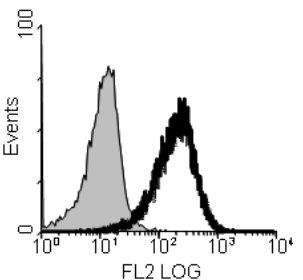

HC4

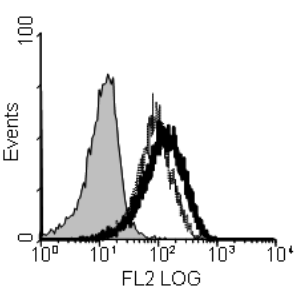

LC3

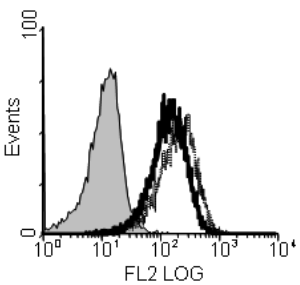

Figure 3-9: Plasma stability of scFv immunoliposomes. Immunoliposomes were incubated with PBS or human plasma at $37^{\circ} \mathrm{C}$ for 4 days and subsequently analyzed for binding to HT1080 FAP ${ }_{\text {mo }}$. grey fill: cells alone, black line: immunoliposomes (10 nmol lipid) incubated with PBS, dashed line: immunoliposomes (10 nmol lipid) incubated with human plasma.

\subsubsection{ScFv' Formats Combining a Cysteine Residue and Hexahistidyl-Tag in the Linker}

In order to reduce the number of additional amino acids within the molecules, two further scFv' variants were generated, with the hexahistidyl-tag incorporated into the peptide linker together with a cysteine residue either at position 1 or 3 (LCH variants) (Figure 3-10).

scFv“ LCH1 V $_{\mathrm{H}}$-ČGGGHHHHHHGGGSA-V scFv‘ LCH3 $V_{H}$-GGC̈GHHHHHHGGGSA-V
LCH1

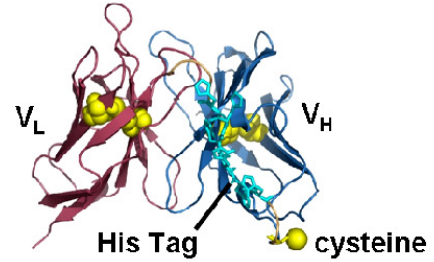

LCH3

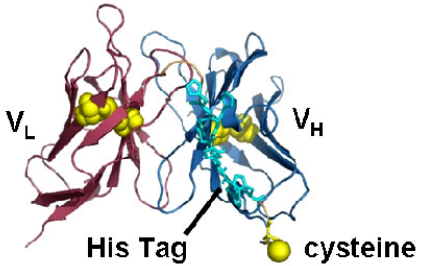

Figure 3-10: Composition of scFv' ${ }^{`} \mathrm{CH}$ variants. Sulfur atoms of the cysteine residues are shown as spheres. Linker sequences and model structure of the LCH constructs. Structures were visualized with PyMol (http://www.pymol.org).

Both constructs could be expressed in soluble form in bacteria and purified by IMAC (yield: $0.3 \mathrm{mg} / \mathrm{l})$. SDS-PAGE and immunoblot analysis revealed for both scFv' variants a major band of $26 \mathrm{kDa}$ under reducing and nonreducing conditions (Figure 3-11). For both constructs dimers with an apparent molecular mass of $56 \mathrm{kDa}$ were detected under nonreducing conditions. Also some proteolytic cleavage products were observed. 
a)

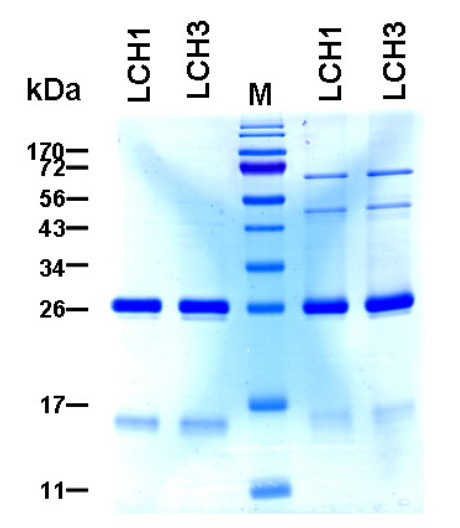

b)

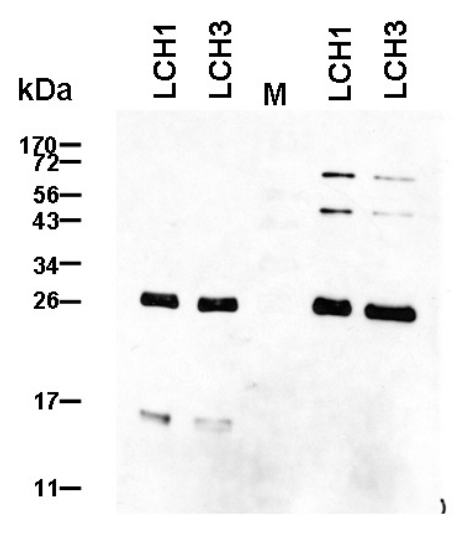

Figure 3-11: SDS-PAGE and immunoblot analysis of purified scFv' LCH variants. (a) Coomassiestained SDS-PAGE ( $2 \mu \mathrm{g} / \mathrm{lane})$ and (b) immunoblot experiment ( $1 \mu \mathrm{g} / \mathrm{lane})$ of purified LCH1 and LCH3 analyzed under reducing (left side of marker) or nonreducing (right side of marker) conditions. Detection was performed with an anti-His-tag antibody.

In size exclusion chromatography by HPLC, scFv' LCH constructs eluted in one main peak (Figure 3-12 a). The LCH1 variant eluted with an apparent molecular mass of $16 \mathrm{kDa}$ while the LCH3 construct with $19 \mathrm{kDa}$, which are much lower than the calculated mass of 26.8 kDa. Minor peaks of higher molecular mass indicate dimers. The melting point of the LCH variants was determined to be in the range of $56-58^{\circ} \mathrm{C}$ (Figure $3-12 \mathrm{~b}$ ).

a)

LCH1

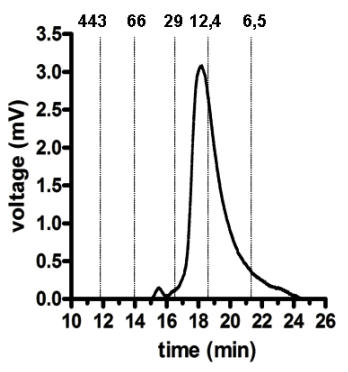

b)

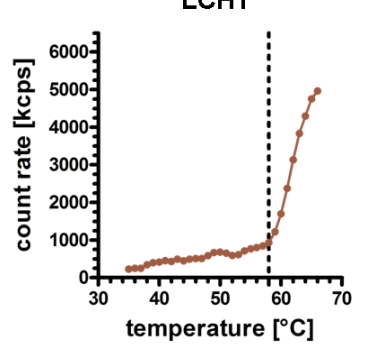

LCH3

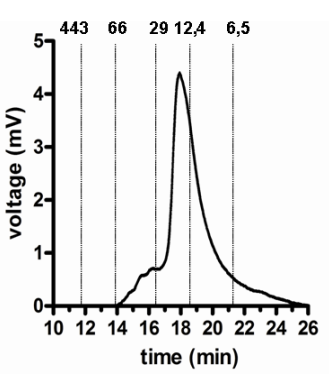

LCH3

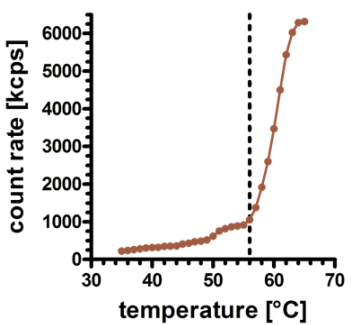

Figure 3-12: Size exclusion chromatography and thermal stability. (a) ScFv' LCH variants were analyzed by HPLC size exclusion chromatography using a BioSepSec-2000 column. Peak positions of standard proteins are indicated. (b) Melting curves of the $\mathrm{LCH}$ variants were determined with $1^{\circ} \mathrm{C}$ intervals using ZetaSizer NanoZS. 
The binding of the LCH variants to FAP-expressing cells was determined by flow cytometry. Both LCH variants showed strongly reduced flow cytometry signals (Figure 3-13). This is probably due to the reduced detection of these variants with the anti-His-tag antibody. The two LCH variants showed no binding to FAP-negative cells (data not shown).
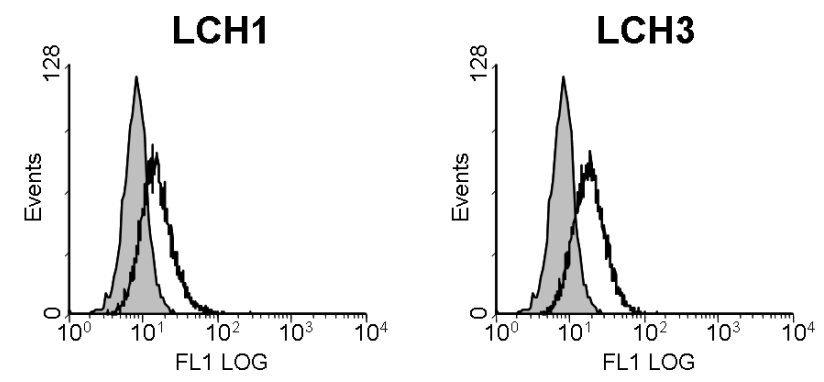

Figure 3-13: Binding analysis of LCH scFv' variants to FAP-expressing cells by flow cytometry. ScFv' variants $(10 \mu \mathrm{g} / \mathrm{ml})$ were incubated with $250000 \mathrm{HT} 1080-\mathrm{FAP}_{\text {mo }}$ cells. Grey fill: cells alone; black line: scFv' molecules (10 $\mathrm{gg} / \mathrm{ml}$, detection: FITC-conjugated anti-His-tag antibody (1:500)).

Although detection of cell bound scFv' $\mathrm{LCH}$ constructs was reduced, immunoliposomes generated from the two scFv' 36 variants LCH1 $(103 \pm 2.1 \mathrm{~nm})$ and LCH3 (106 $\pm 1.7 \mathrm{~nm})$ showed a strong and specific binding to a FAP-positive cells line and no binding to wild-type cells (Figure 3-14). Binding intensity was similar to that observed for the LC variants (Figure 3-7). Determined sizes of $\mathrm{LCH}$ immunoliposomes was similar to those measured for immunoliposomes generated from other scFv' variants (Table 3).
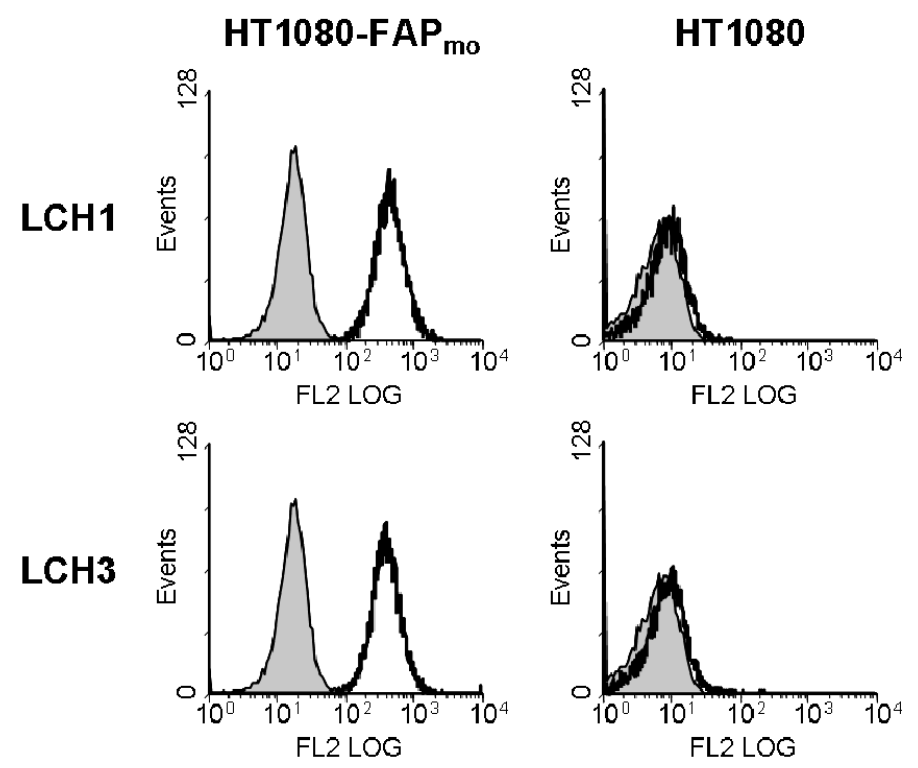

Figure 3-14: Binding of LCH1 and LCH3 immunoliposomes to FAP-expressing cells. Micellecoupled ScFv's were inserted into preformed PEGylated liposomes (2 mol\% micellar lipids) and analyzed for binding to FAP-expressing cells (HT1080-FAP ${ }_{\text {mo }}$ ) or to wild-type HT1080 cells. Grey fill: cells alone, black line: cells incubated with liposomes at $10 \mathrm{nmol}$ lipid per 250.000 cells In order to analyze whether binding to cells under physiological conditions leads to an uptake 
of the scFv immunoliposomes by receptor-mediated endocytosis (Baum et al., 2007), HT1080 cells were incubated with scFv36 immunoliposomes in cell culture medium for $6 \mathrm{~h}$ at $37^{\circ} \mathrm{C}$. The immunoliposomes were fluorescently labeled with $\mathrm{DiO}$. The internalization study of scFv36 LCH3 immunoliposomes is exemplarily shown in Figure 3-15. Binding to the cell surface and uptake of immunoliposomes to FAP-expressing cells could clearly be seen, whereas no uptake was observed with FAP-negative cells.
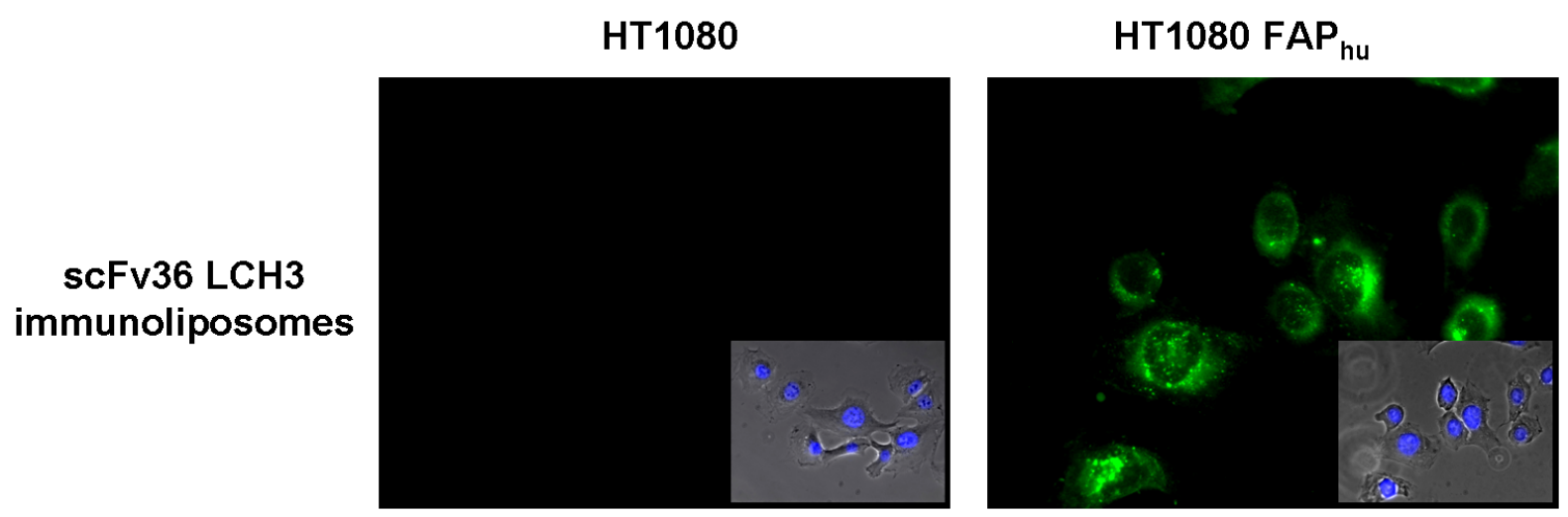

Figure 3-15: Selective uptake of immunoliposomes in FAP expressing cells. Fluorescence microscopy images of scFv36 LCH3 immunoliposomes on FAP-negative (HT1080) and FAP-positive $\left(\mathrm{HT} 1080-\mathrm{FAP}_{\mathrm{hu}}\right)$ cell lines after $6 \mathrm{~h}$ at $37^{\circ} \mathrm{C}$. Immunoliposomes $(25 \mathrm{nmol})$ are labeled with $\mathrm{DiO}$ (green), nuclei are stained with DAPI (blue), cells are shown in bright field images. 


\subsection{Targeted Lipid-Coated Nanoparticles: Delivery of Tumor Necrosis Factor-Functionalized Particles to Tumor Cells}

Amino-functionalized silica nanoparticles decorated with tumor necrosis factor (TNF), termed nanocytes ${ }^{\circledR}$ (Bryde et al., 2005) are known for a potent activation of the two TNF-receptors and an effective induction of apoptosis. Thus, TNF-nanocytes are potentially useful therapeutics. However the therapeutic utilization is limited due to the lack of selectivity of the TNF nanocytes for target tissues and systemic toxicity as possible consequence. Encapsulation of nanoparticles within liposomes has been proposed as a method to enhance the plasma stability (Al-Jamal \& Kostarelos 2007; De Miguel et al., 2000; Senarath-Yapa et al., 2007; van Schooneveld et al., 2008) and should also be capable of shielding the organism from the unspecific action of protein-decorated nanoparticles. Additionally, an incorporation of ligands to the lipid surface should further allow an active targeting to tissues and cells (Torchilin, 2007). In this work polystyrene particles were used to establish methods for the generation of single-chain TNF-functionalized nanoparticles.

\subsubsection{Establishment of Methods for Lipid Coating of Nanoparticles}

In a first set of experiments, two standard protocols were established for lipid coating of amino-functionalized polystyrene particles (NSL-PMI). A schematic diagram of the both established method for lipid coating is presented in Figure 3-17.

\subsubsection{Lipid-Coating by Extrusion}

For this method the nanoparticles were coated with lipids by adding particles to a lipid film composed of EPC, cholesterol and $\mathrm{mPEG}_{2000}$-DSPE (2 mol\% or $5 \mathrm{~mol} \%$ ) followed by subsequent extrusion through $200 \mathrm{~nm}$ membranes at $60^{\circ} \mathrm{C}$. To separate lipid-coated particles $(L P)$ from empty liposomes $(L)$ and non-coated particles $(P)$ a density centrifugation through a 6-15\% sucrose gradient was performed (Figure 3-16). Nanoparticles alone migrated to the bottom of the gradient, whereas liposomes were located near the top of the sucrose gradient. The lipid-coated nanoparticle (LP) accumulated between liposomes and free particles, thus LP exhibited an intermediate density and could be clearly separated from particles and liposomes. After treatment with $2 \%$ Triton X-100 the LP and liposome bands could not be observed. A single band corresponding to free particles was seen, thus confirming the lipid coating of particles. 


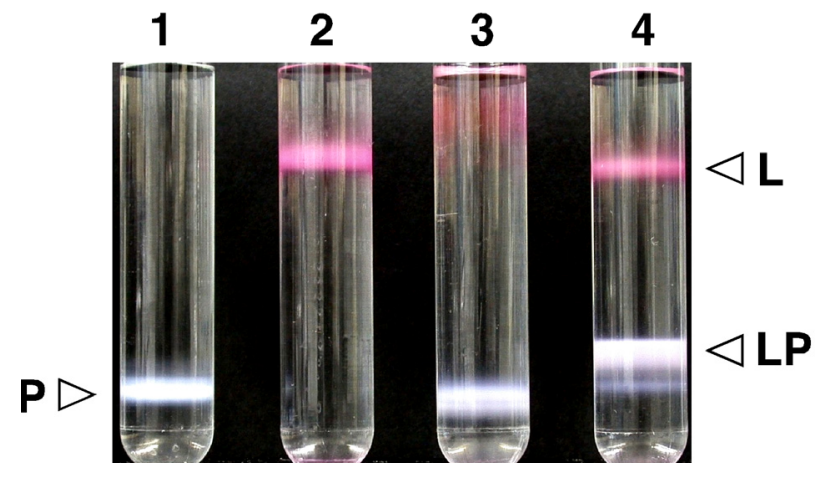

Figure 3-16: Sucrose density gradient. Separation of lipid-coated particles (LP) from liposomes (L) and particles $(P)$. Particle preparation were overlayed with a sucrose gradient $(6-15 \%$ in water) and centrifuged for $20 \mathrm{~h}$ at $68000 \mathrm{~g}$ at $4^{\circ} \mathrm{C}$. 1, $200 \mu \mathrm{g}$ amino-functionalized polystyrene particles (NSLPMI); 2, 2 nmol liposomes; 3, $200 \mu \mathrm{g}$ lipid-coated particle preparation incubated with $2 \%$ Triton X-100; 4, $200 \mu \mathrm{g}$ lipid-coated particle preparation.

\subsubsection{Lipid-Coating by Sonification}

Lipid-coating can alternatively performed by sonification of particles with preformed liposomes composed of EPC, cholesterol and $\mathrm{mPEG}_{2000}$-DSPE (2 mol\% or $5 \mathrm{~mol} \%$ ). The liposomes were prepared with the film hydration method and extruded through $100 \mathrm{~nm}$ membranes. The coating with lipids was then induced by sonification for 20 min at $60{ }^{\circ} \mathrm{C}$. Lipid-coated particles were separated from empty liposomes by a centrifugation step but can also separated by using a sucrose gradient centrifugation. 


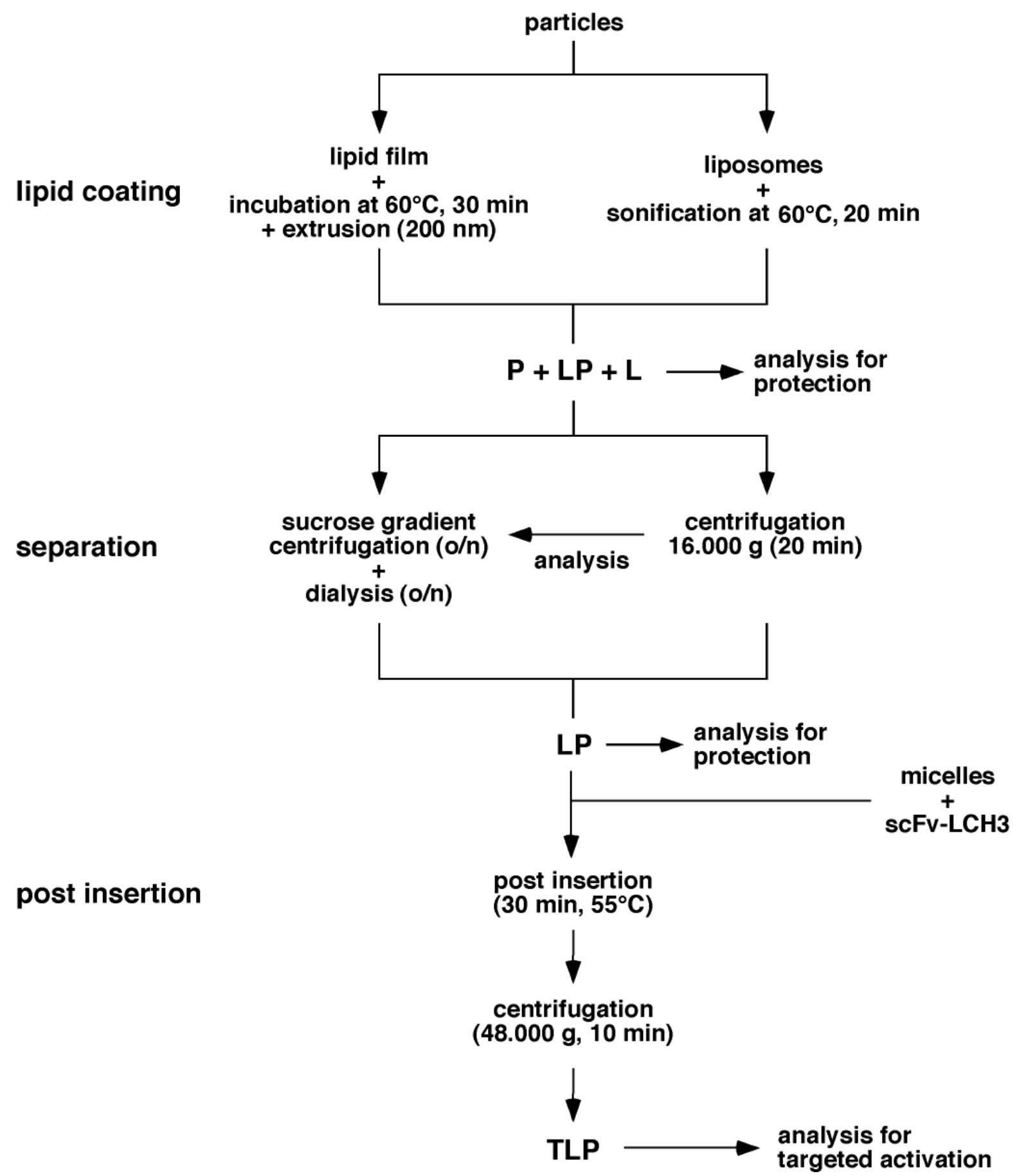

Figure 3-17: Strategy to generate targeted lipid-coated particle. Lipid-coated amino-functionalized polystyrene particles (LP) are produced by adding the particles either to a lipid film $\left(\mathrm{EPC} / \mathrm{Chol} / \mathrm{mPEG}_{2000}-\mathrm{DSPE}=6.5: 3.0 .5\right)$ and subsequent extrusion through a $200 \mathrm{~nm}$ membrane at 60 ${ }^{\circ} \mathrm{C}$ or to liposomes $\left(\mathrm{EPC} / \mathrm{Chol} / \mathrm{mPEG}_{2000}\right.$-DSPE $\left.=6.5: 3.0 .5,100 \mathrm{~nm}\right)$ followed by sonification for 20 $\min$ at $60^{\circ} \mathrm{C}$. Lipid-coated particles (LP) are separated from lipids and liposomes either by sucrose density centrifugation and subsequently dialysis of the LP fraction is against Hepes buffer or by centrifugation for $10 \mathrm{~min}$ at $40000 \mathrm{~g}$. In parallel, the antibody fragment is coupled through the sulfhydryl group of a genetically introduced cysteine residue to Mal-PEG ${ }_{2000}$-DSPE micelles and inserted into LP by incubation for $30 \mathrm{~min}$ at $55^{\circ} \mathrm{C}$ resulting in TLP. Unconjugated antibody fragments are separated from TLP by centrifugation for $10 \mathrm{~min}$ at $48000 \mathrm{~g}$. 


\subsubsection{Targeted Lipid-Coated Nanoparticles (TLP)}

Targeted lipid-coated nanoparticles (TLP) were prepared by coupling scFv' 36 LCH3 to Mal$\mathrm{PEG}_{2000}$-DSPE micelles and following insertion into LP (5 mol\% micellar lipid in respect to LP lipid) for $30 \mathrm{~min}$ at $55^{\circ} \mathrm{C}$. Non-coupled scFv molecules were subsequently removed by centrifugation (FIGURE). The size determined by dynamic light scattering intensity of LP and TLP was dependent on the amount of $\mathrm{mPEG}_{2000}-\mathrm{DSPE}$ (Table 4). The presence of $2 \mathrm{~mol} \%$ mPEG resulted in an increase in size of TLP in the range of 91-98 nm, while TLP containing $5 \mathrm{~mol} \%$ mPEG showed an increase between 30-35 nm. This was independent of functionalization of the particle surface.

Table 4: Particle characterization

P, particles; LP, lipid-coated particles; TLP, targeted lipid-coated particles

\begin{tabular}{|c|c|c|c|c|}
\hline Formulation & $\begin{array}{c}\text { mPEG-DSPE } \\
\text { (mol \%) }\end{array}$ & $\begin{array}{c}\text { ScFv-Mal-PEG-DSPE } \\
(\mathrm{mol} \%)\end{array}$ & $\begin{array}{l}\text { Size } \\
(\mathrm{nm})\end{array}$ & PDI \\
\hline \multicolumn{5}{|c|}{ non-functionalized particles based SL-PMI } \\
\hline $\mathrm{P}$ & - & - & $174 \pm 3.5$ & 0.18 \\
\hline LP & 2 & - & $241 \pm 3.6$ & 0.20 \\
\hline LP & 5 & - & $176 \pm 4.1$ & 0.29 \\
\hline TLP & 2 & 5 & $272 \pm 9.9$ & 0.30 \\
\hline TLP & 5 & 5 & $209 \pm 4.0$ & 0.25 \\
\hline \multicolumn{5}{|c|}{ amino-functionalized particles based on NSL-PMI } \\
\hline$P$ & - & - & $186 \pm 1.4$ & 0.11 \\
\hline LP & 2 & - & $251 \pm 10.0$ & 0.29 \\
\hline LP & 5 & - & $210 \pm 2.4$ & 0.18 \\
\hline TLP & 2 & 5 & $277 \pm 18.0$ & 0.33 \\
\hline TLP & 5 & 5 & $216 \pm 1.4$ & 0.14 \\
\hline
\end{tabular}

\subsubsection{Binding Studies of Targeted Lipid-Coated Particles}

The TLP preparations were analyzed for binding to FAP-expressing (HT1080 FAP hu $)$ and FAP-negative (HT1080 wt) cells. Binding of particles, LP and TLP was detected through a green fluorescence dye (perylene monoimide, PMI) incorporated into polystyrene particles. Both, non-functionalized (SL-PMI) as well as amino-functionalized (NS-PMI) particles showed unspecific binding to $\mathrm{HT}_{1080} \mathrm{FAP}_{\mathrm{hu}}$ and HT1080 wt cells (Figure 3-18). LP prepared from SL-PMI with coating lipids containing 2 mol\% mPEG $2000-D S P E$ showed a completely reduction of non-specific binding to FAP-negative and FAP-positive cells. TLP exhibited a specific binding to HT1080 $\mathrm{FAP}_{\text {hu }}$ cells after insertion of scFv-coupled Mal-PEG ${ }_{2000}$-DSPE (5 mol\%). Lipid coating containing $5 \mathrm{~mol} \% \mathrm{mPEG}_{2000}-\mathrm{DSPE}$ revealed an unspecific binding of 
LP to wild-type and FAP-expressing cells, which was completely reduced for TLP on FAPnegative cells.

HT1080 wt
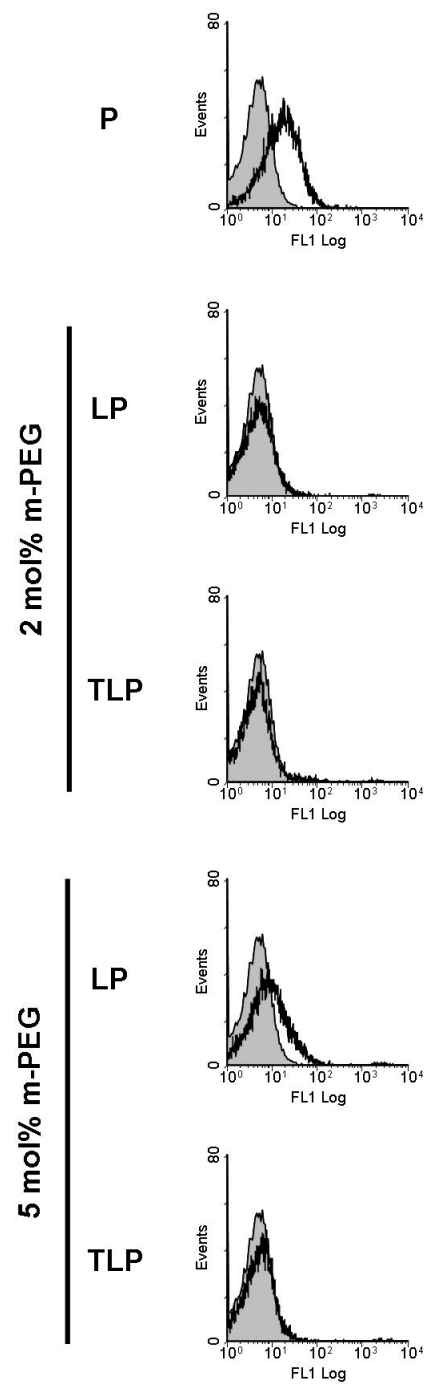

not-functionalized particles
HT1080 wt
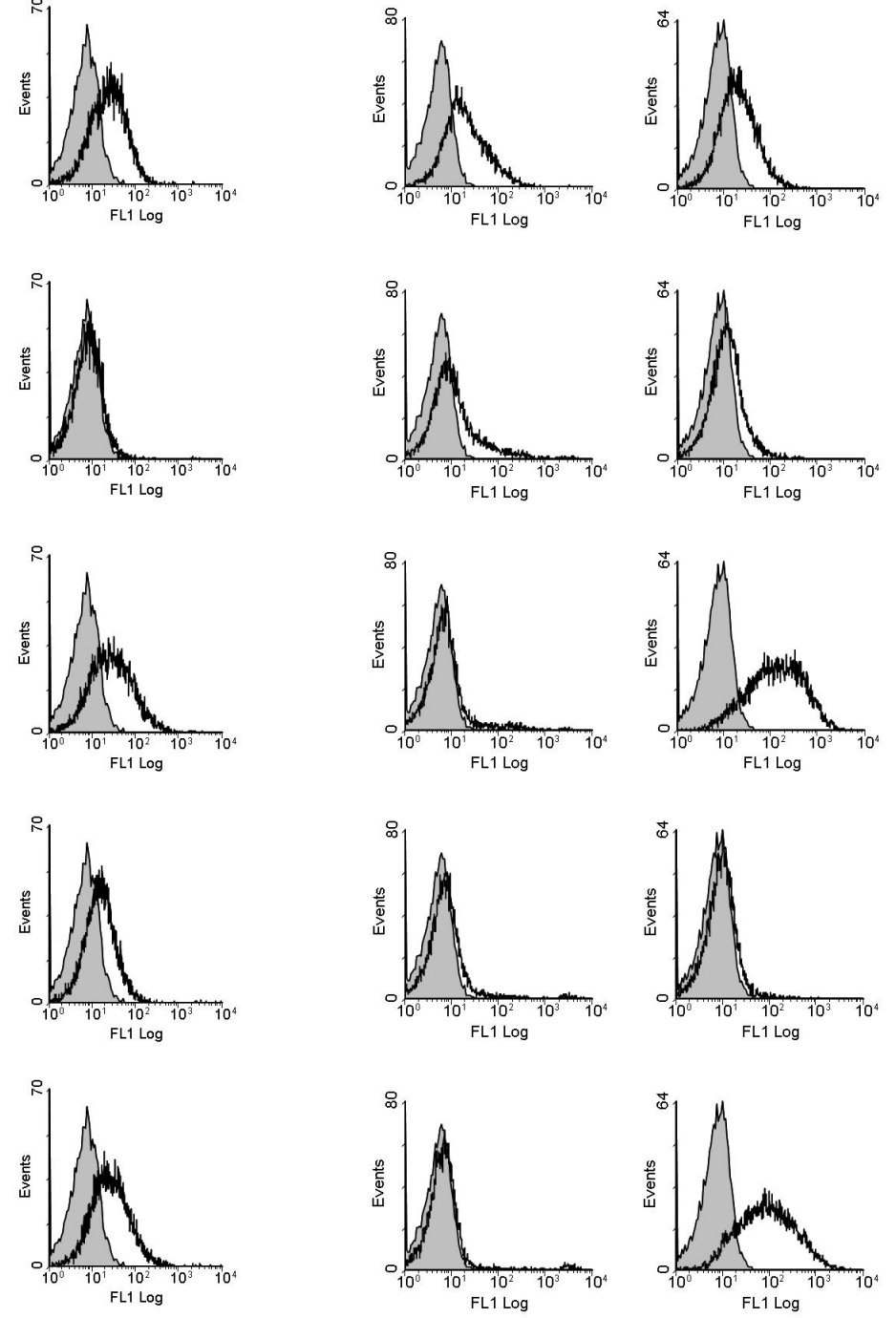

amino-functionalized particles

Figure 3-18: Flow cytometry analysis of $P$, LP, and TLP prepared from non-functionalized (SLPMI) or amino-functionalized (NSL-PMI) $P$ for binding to FAP ${ }^{-}$HT1080 wild-type (wt) cells or $\mathrm{FAP}^{+} \mathrm{HT} 1080 \mathrm{FAP}_{\text {hu }}$ cells. LP and TLP were prepared with 2 or $5 \mathrm{~mol} \% \mathrm{mPEG}_{2000}-\mathrm{DSPE}$ and insertion of $5 \mathrm{~mol} \%$ micellar scFv-coupled Mal-PEG ${ }_{2000}$-DSPE lipid. Grey fill: cells alone, black line: cells incubated with particle preparations at $3 \mu \mathrm{g} / 250000$ cells).

Lipid coated amino-functionalized (NS-PMI) particles containing 2 mol\% mPEG ${ }_{2000}-\mathrm{DSPE}$ showed a slight binding to HT1080 wild-type and HT1080 FAP hu cells, which was further reduced for anti-FAP TLP on wild-type cells and showed a strong binding on FAP-positive cells. LP composed of coating lipids with 5 mol\% $\mathrm{mPEG}_{2000}$-DSPE possessed a marginal unspecific binding to HT1080 cells. The residual binding to HT1080 wild-type cells was 
completely reduced for TLP (5 mol\% MalPEG $\left.{ }_{2000}-D S P E\right)$, which exhibited a strong and specific binding to FAP-expressing cells. Binding of TLP, prepared from SL-PMI or NS-PMI, to FAP-positive cells was not influenced by the concentration of $m P E G_{2000}$-DSPE.

LP and anti-FAP TLP, prepared from NS-PMI particles, were further analyzed on FAPnegative Kym-1 cells (Figure 3-19). A strong binding of uncoated amino-functionalized particles could be observed, whereas lipid-coated and targeted lipid-coated particles showed no or only very weak binding to Kym-1 cells.
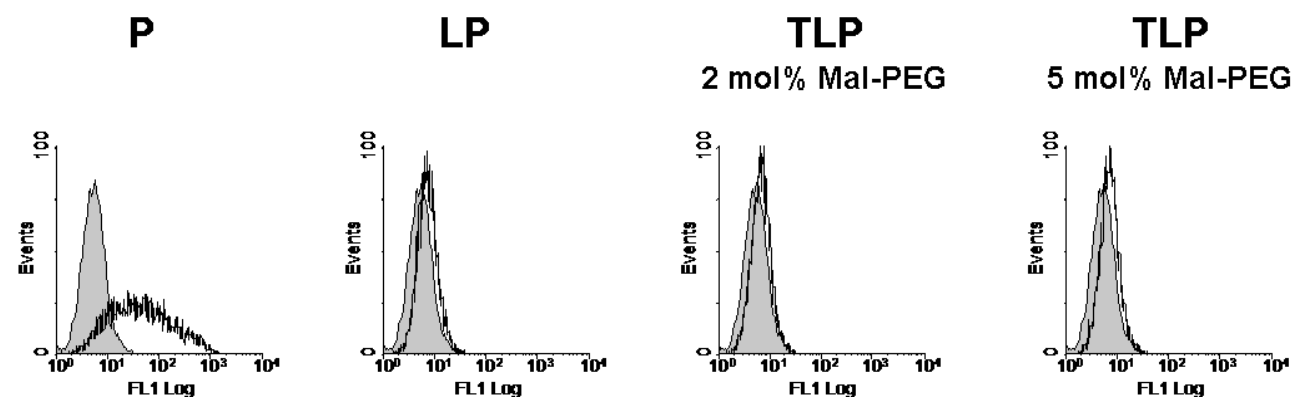

Figure 3-19: Flow cytometry analysis of $P$, LP and TLP prepared from amino-functionalized (NSL-PMI) $\mathbf{P}$ to $\mathrm{Kym}-1$ cells. LP and TLP were prepared with 5 mol\% $\mathrm{mPEG}_{2000}$-DSPE and insertion of 2 or $5 \mathrm{~mol} \%$ micellar scFv-coupled Mal-PEG $2000-\mathrm{DSPE}$ lipid. Grey fill: cells alone, black line: cells incubated with particle preparations at $3 \mu \mathrm{g} / 250000$ cells.

The amount of antibody molecules incorporated into TLP depends on the concentration of inserted Mal-PEG ${ }_{2000}$-DSPE. In order to determine the optimal amount required for strong binding to target cells a titration of scFv-coupled Mal-PEG ${ }_{2000}-\mathrm{DSPE}$ lipid was performed. Therefor LP prepared from amino-functionalized green-fluorescing particles (NSL-PMI) containing 5 mol\% $\mathrm{mPEG}_{2000}$-DSPE were used and between 0.2 to $5 \mathrm{~mol} \%$ scFv-coupled micellar lipid were inserted into the lipid coat. The resulting TLP showed an increasing binding to $\mathrm{HT} 1080 \mathrm{FAP}_{\text {hu }}$ cells with increasing amounts of inserted scFv (Figure 3-20). Also, TLP containing the highest concentration of inserted Mal-PEG ${ }_{2000}-\mathrm{DSPE}$ lipid (5 mol\%) showed the lowest unspecific binding to FAP-negative cells, indicating that Mal-PEG ${ }_{2000^{-}}$ DSPE contributes in reduction nonspecific binding.

In the following, experiments were performed with preparation of TLP containing $5 \mathrm{~mol} \%$ Mal-PEG ${ }_{2000}$-DSPE inserted into LP coated with 5 mol\% $\mathrm{mPEG}_{2000}$-DSPE lipid. 


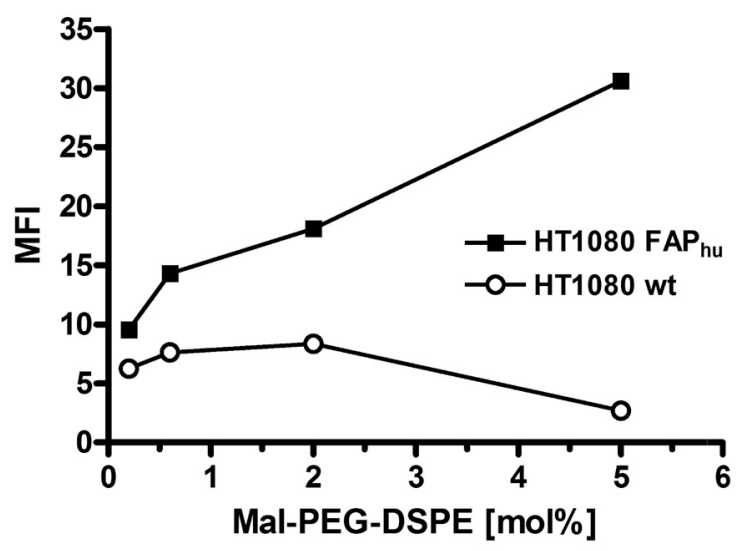

Figure 3-20: Dependence of target cell binding on inserted scFv coupled to Mal-PEG ${ }_{2000}-\mathrm{DSPE}$. LP and TLP were prepared from amino-functionalized green-fluorescing $P$ (NSL-PMI). Varying amounts of scFv-coupled Mal-PEG 2000 -DSPE $(0.2-5$ mol\%) were inserted into LP containing $5 \mathrm{~mol} \%$ mPEG-DSPE and resulting TLP were analyzed by flow cytometry for binding to HT1080 wild-type and HT1080 FAP hu cells. $3 \mu \mathrm{g}$ particles/250 000 cells.

In order to analyze the lipid coating of particles further flow cytometry analysis were performed. Therefor amino-functionalized, red-fluorescing particles (NSL-Lum, $274 \mathrm{~nm}$ ) containing Lumogen F Red and lipids containing the green fluorescent dye DiO were used.

a)

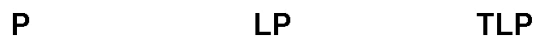

HT1080 wt
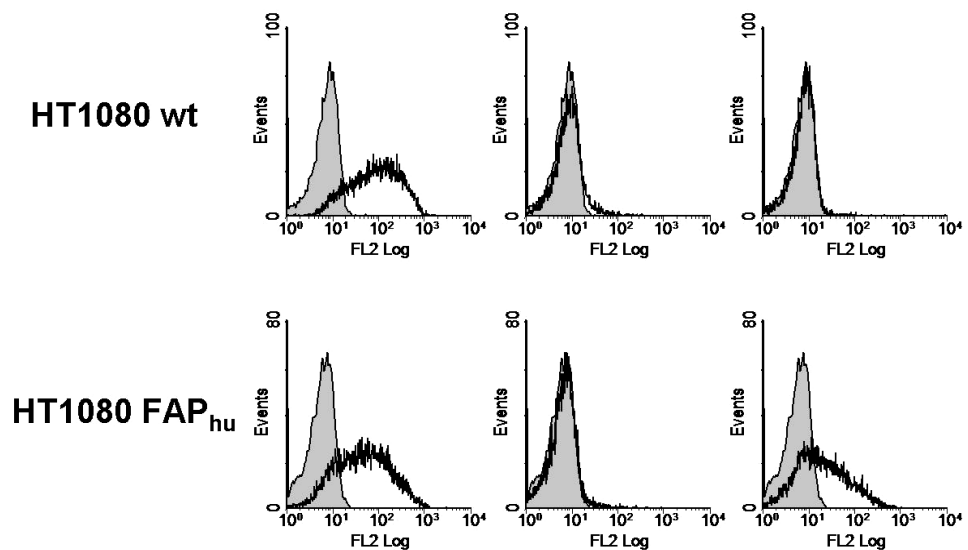

b)

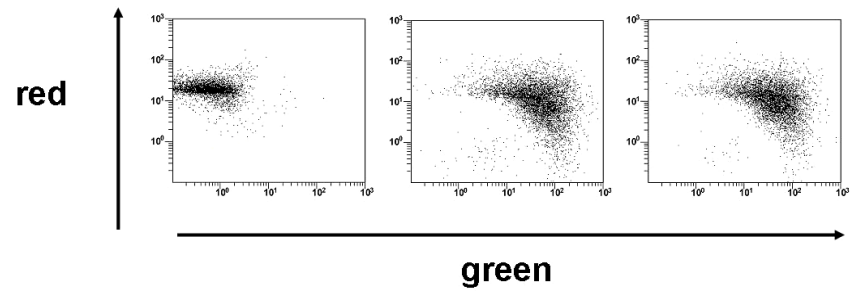

Figure 3-21: Analysis of lipid coating of particles. LP and TLP were prepared from aminofunctionalized red-fluorescing P (NSL-Lum). (a) Flow cytometry analysis for binding to HT1080 wt and HT1080 $\mathrm{FAP}_{\text {hu }}$ cells. Grey fill: cells alone, black line: cells incubated with particle preparations at 3 $\mu \mathrm{g} / 250000$ cells. b) Flow cytometry analysis of $\mathrm{P}, \mathrm{LP}$ and TLP showed a red fluorescence of $\mathrm{P}$, while fluorescence of LP and TLP was shifted from red toward green fluorescence. 
These P, LP and TLP exhibited the same cell binding behavior (Figure 3-21 a) as for the other green-fluorescing particles shown (Figure 3-18): A strong and unspecific binding to both cell lines of uncoated $P$, which is nearly completely reduced after lipid-coating, while TLP showed specific binding only on FAP-expressing cells. Additionally flow cytometry revealed a strong red fluorescence of particles, whereas LP and TLP showed red and green fluorescence (Figure 3-21 b), demonstrating for co-localizations of lipids (green) and particles (red).

To verify the specific binding of TLP to FAP and not to any other molecule on the cell surface, binding to $\mathrm{HT} 1080 \mathrm{FAP}_{\mathrm{hu}}$ should be blocked by addition of soluble scFv36 $(10 \mu \mathrm{g} / \mathrm{ml})$. As a negative control the cells were also preincubated with an irrelevant scFv (A5) directed against another antigen (endoglin), which is expressed on HT1080 cells. Binding of TLP prepared from NSL-PMI was completely blocked by scFv36, while no blocking was seen with the irrelevant scFv (Figure 3-22). Binding of uncoated particles and LP was not affected by both antibody molecules.

$\mathbf{P}$
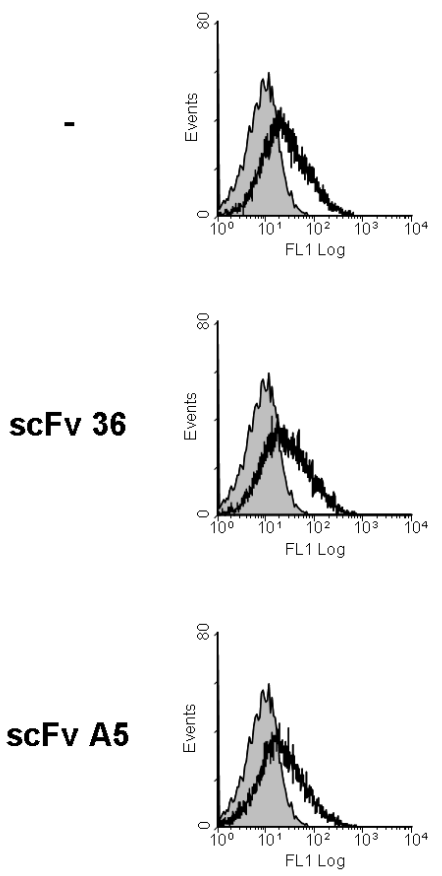

LP
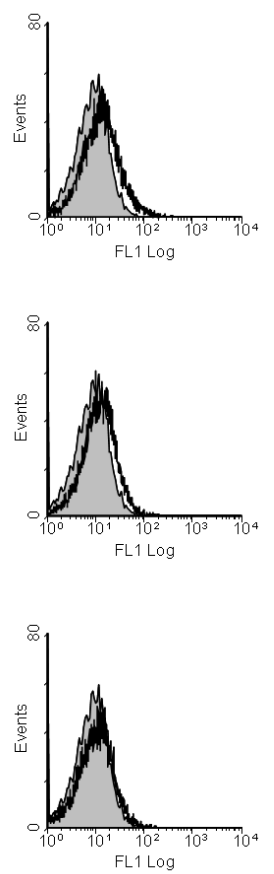

TLP
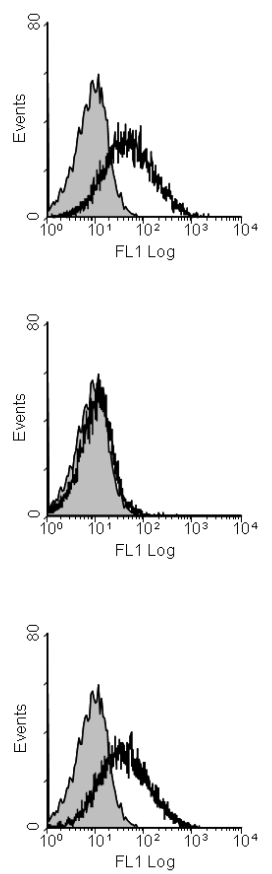

Figure 3-22: Selectivity of binding of TLP to HT1080 FAP $_{\text {hu }}$ cells. TLP were prepared from aminofunctionalized green-fluorescing P (NSL-PMI). Binding of TLP to HT1080 FAP hu cells is blocked by 10 $\mu \mathrm{g}$ of soluble anti-FAP scFv 36 but not an irrelevant scFv (A5), while nonspecific binding of $P$ is not affected. Grey fill: cells alone, black line: cells incubated with P, LP or TLP and ScFv as indicated. 
To verify stability of TLP under physiological conditions, TLP prepared from aminofunctionalized green-fluorescing $P$ (NSL-PMI) were incubated with human plasma or in PBS at $37{ }^{\circ} \mathrm{C}$. No reduction in specific binding to $\mathrm{HT} 1080 \mathrm{FAP}_{\mathrm{hu}}$ was observed after $24 \mathrm{~h}$ of incubation with plasma. Additionally, no statistically significant difference in binding to FAPexpressing cells after incubation with plasma in comparison to incubation in PBS at $37^{\circ} \mathrm{C}$ was seen. Above all, no increase in nonspecific binding of TLP to HT1080 wild type cells could be observed, neither after incubation with plasma nor incubation in PBS. These results indicate that TLP are stable under physiological conditions for at least 24 hours with respect to antigen-binding activity.

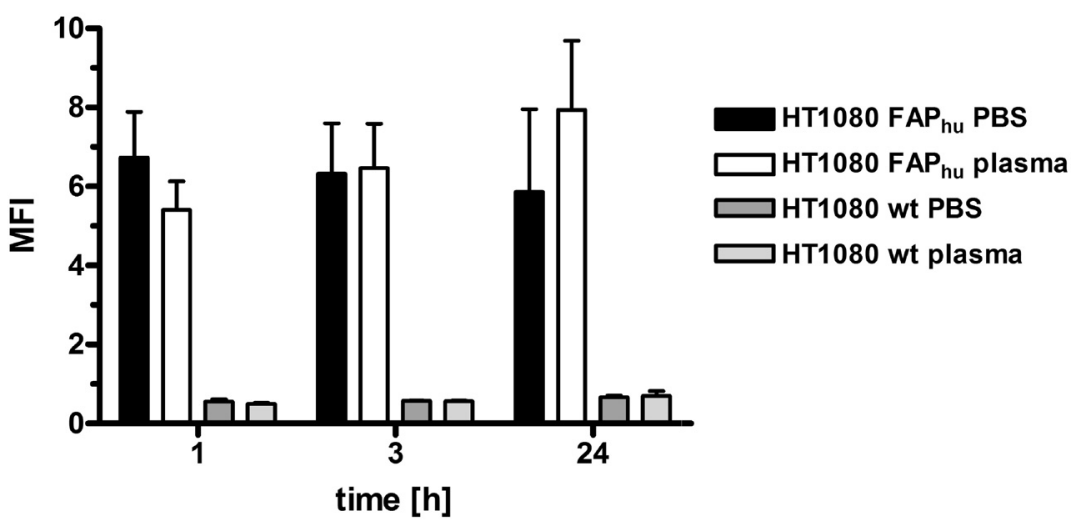

Figure 3-23: Plasma stability of TLP. TLP prepared from amino-functionalized green-fluorescing $P$ (NSL-PMI) were incubated with either PBS or human plasma at $37^{\circ} \mathrm{C}$ for 1,3 , and $24 \mathrm{~h}$ and subsequently analyzed for binding to HT1080 FAP $\mathrm{Fu}_{\mathrm{h}}$ and HT1080 wt, respectively $(n=3)$.

\subsubsection{Internalization Studies by Fluorescence Microscopy}

In order to confirm the results obtained from flow cytometry analysis of uncoated particles, LP and TLP internalization studies by fluorescence microscopy were performed. HT1080 cells were incubated with preparations from amino-functionalized, red-fluorescing particles (NSL-Lum) under permanent rolling in culture medium for $6 \mathrm{~h}$ at $37^{\circ} \mathrm{C}$ and were subsequently fixed. The unspecific and strong internalization of uncoated particles into both HT1080 cell lines could clearly be seen. The particles seem to be accumulated in vesiculare structures. LP showed no or only marginal unspecific binding or uptake to both cell lines, respectively. Also anti-FAP TLP demonstrated a negligible binding to wild-type cells. Importantly TLP exhibited a significantly fluorescence signal only on HT1080 FAP hu cells, mostly of internalization into vesiculare structures but also binding on the cell surface. 

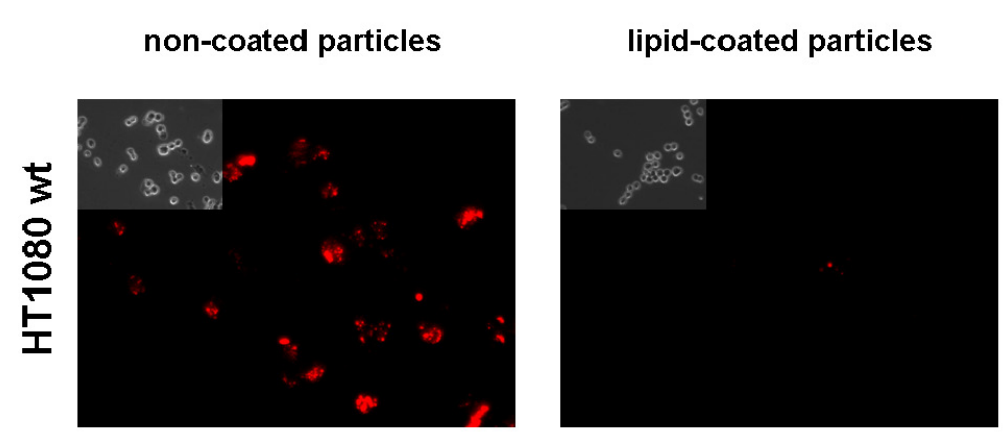

anti-FAP targeted lipid-coated particles
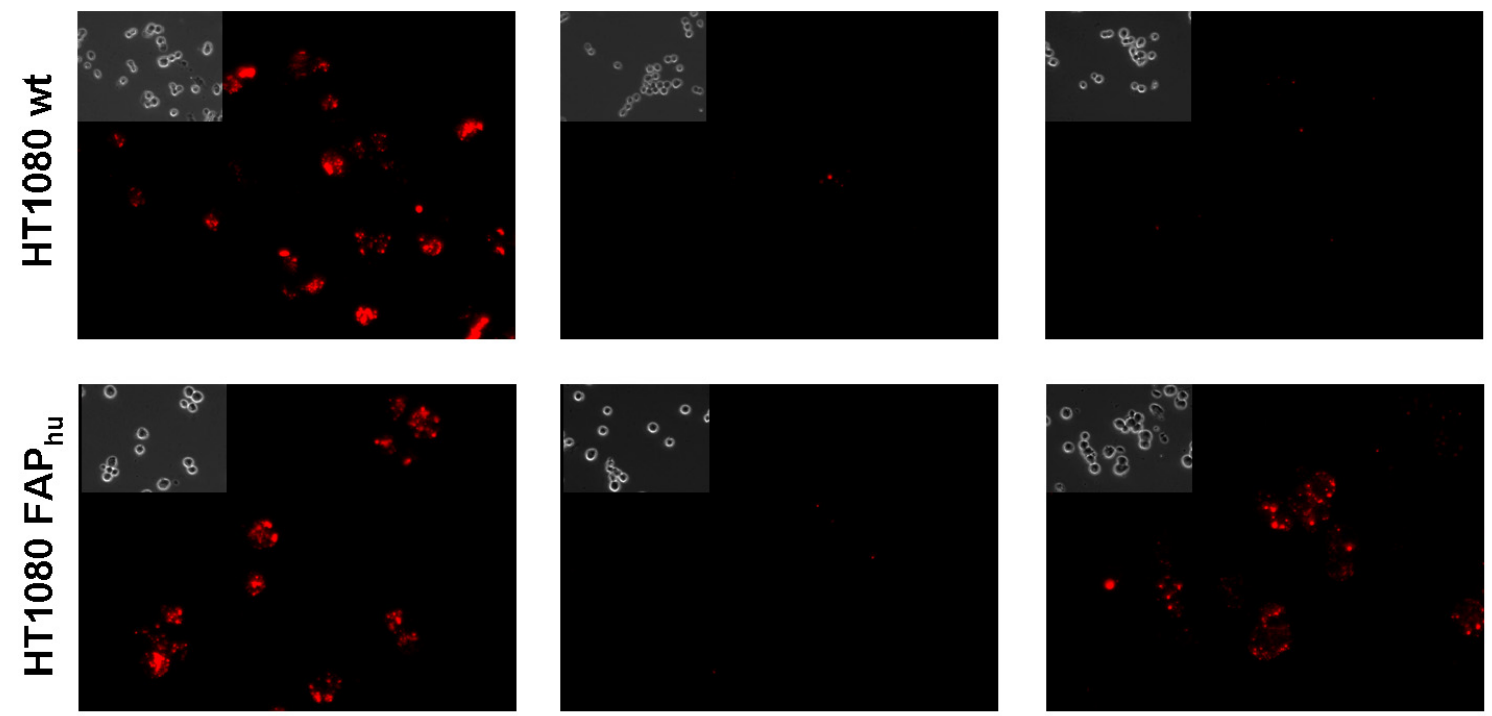

Figure 3-24: Internalization studies of particle preparations. Fluorescence microscopy images particles, LP and anti-FAP TLP on FAP-negative (HT1080 wt) and FAP-positive (HT1080-FAP ${ }_{\text {hu }}$ ) cell lines after $6 \mathrm{~h}$ at $37^{\circ} \mathrm{C}$. Particles $(3 \mu \mathrm{g})$ contain a red fluorescent dye; cells are shown in bright field images.

\subsubsection{Pharmacokinetic Properties}

To analyze the pharmacokinetic properties of $P$ and LP in mice, amino-functionalized particles containing an IR-dye (IR iodide 780) were used. LP were prepared with $5 \mathrm{~mol} \%$ mPEG 2000 -DSPE.

Pharmacokinetics was determined after a single dose (300 $\mu \mathrm{g})$ i.v. injection into CD1 mice. All particle preparations showed biphasic elimination from circulation. The LP were cleared from circulation with an approximately 8.9 increased initial half-life ( $\left.t_{1 / 2} \alpha\right)$ compared to uncoated $P$, while terminal half-life $\left(t_{1 / 2} \beta\right)$ was increased by factor 1.5 . Compared to $P$ the area under the curve ( $\left.\mathrm{AUC}_{0-24 h}\right)$ was increased 1.8 for LP (Figure 3-25, Table 5). 


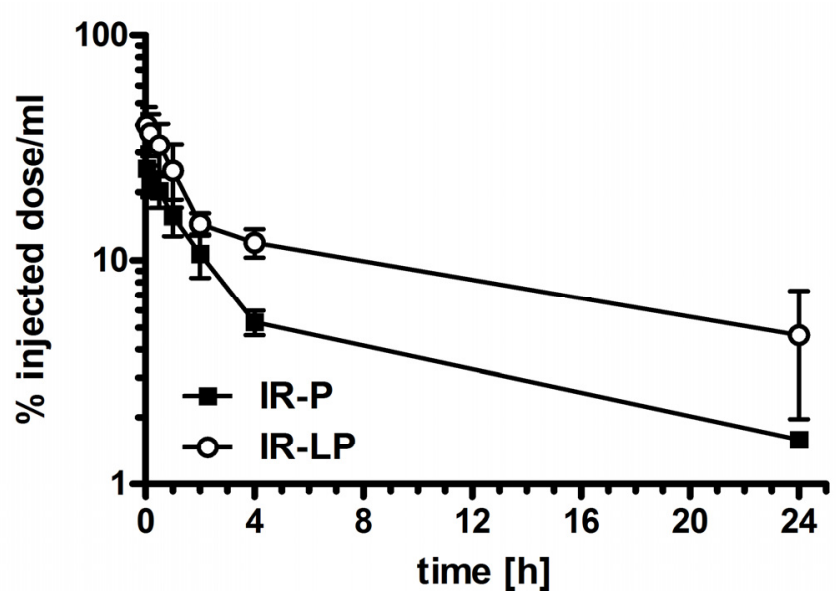

Figure 3-25: Pharmacokinetic properties. $300 \mu \mathrm{g}$ of $\mathrm{P}$ and LP prepared from amino-functionalized IR-P were injected i.v. into CD1 mice. Serum concentrations of particle preparations were determined at different time points by determination particle concentration by means of incorporated IR dye (IR iodide 780 ). Data were normalized related to injected dose.

Table 5: Pharmacokintetic properties. Parameters were determined from data obtained for the first $24 \mathrm{~h}$.

\begin{tabular}{llll}
\hline $\begin{array}{l}\text { Particle } \\
\text { preparation }\end{array}$ & $\begin{array}{l}\mathbf{t}_{1 / 2} \alpha \\
(\min )\end{array}$ & $\begin{array}{l}\mathbf{t}^{1 / 2} \boldsymbol{\beta} \\
(\mathbf{h})\end{array}$ & $\begin{array}{l}\mathrm{AUC}_{\mathbf{0} 24} \\
\left(\% \mathrm{ID}^{*} \mathbf{h}\right)\end{array}$ \\
\hline $\mathrm{P}$ & $53.5 \pm 2.8$ & $9.58 \pm 2.47$ & $136 \pm 47.6$ \\
$\mathrm{LP}$ & $480 \pm 367$ & $14.65 \pm 2.83$ & $243 \pm 4.7$
\end{tabular}

\subsubsection{Generation of scTNF-Nanoparticles}

The bioactive form of the tumor necrosis factor (TNF) has shown to be a compact noncovalently linked homotrimer (Eck et al., 1989; Jones et al., 1990). A TNF derivative with a cysteine/histidine tag (Krippner-Heidenreich et al., 2002) coupled to silica particles (Bryde et al., 2005) showed an effective induction of apoptosis in cells expressing both TNF receptors and acts therefore as TNF homotrimer. However a reversible dissociation of several TNF molecules, which are covalently linked to the particle surface by only one of its monomers, may represent a disadvantage of this TNF derivative. Thus, a further TNF derivate (CysscTNF) was developed providing increased stability and allowing a site-directed conjugation to nanoparticles.

\subsubsection{Cys-scTNF}

ScTNF is a TNF derivative consisting of 3 TNF monomers connected by 2 flexible 12 amino

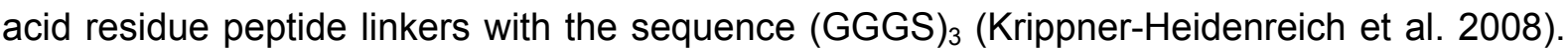
This TNF trimer showed an enhanced stability and anti-tumoral activity. To enable sitedirected coupling, scTNF was further modified by introducing an additional cysteine residue 
at the N-terminal extension (Cys-scTNF, Figure 3-26 a). In SDS-PAGE purified Cys-scTNF migrated with an apparent molecular mass of $50 \mathrm{kDa}$ under reducing conditions (Figure 3-26 b), which is similar to the calculated molecular mass of $54 \mathrm{kDa}$. The bioactivity of Cys-scTNF was tested on Kym-1 cells, a cell line very sensitive to TNF-mediated apoptosis. In this assay, Cys-scTNF $\left(\mathrm{IC}_{50}=70 \mathrm{pg} / \mathrm{ml}\right)$ exhibited a 2.8 fold stronger activity on $\mathrm{Kym}-1$ cells compared to soluble TNF $\left(\mathrm{IC}_{50}=200 \mathrm{pg} / \mathrm{ml}\right.$, Figure 3-26 c).

a)

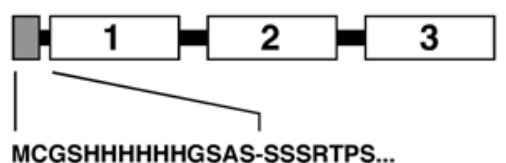

b)

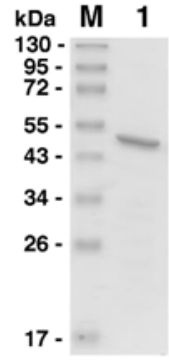

c)

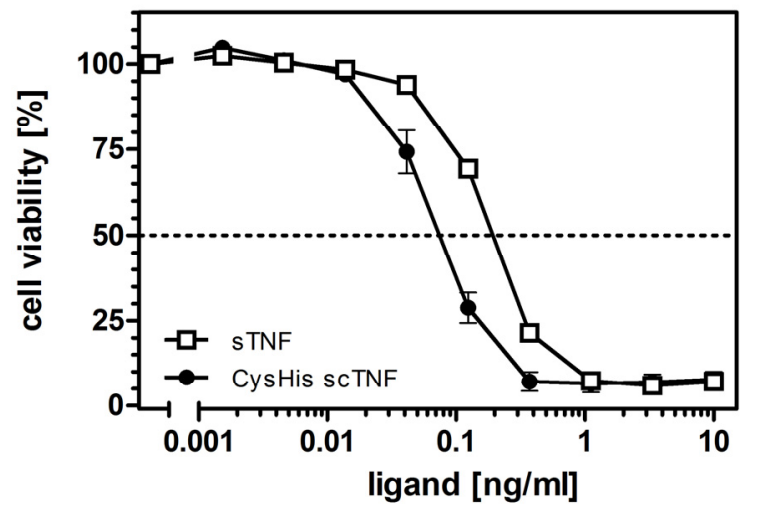

Figure 3-26: Cys-scTNF. (a) Composition of cys-scTNF. The sequence of the N-terminal extension is shown including cysteine residue (position 2) for site-directed coupling. (b) SDS-Page analysis under reducing conditions. Lane 1: $2 \mu \mathrm{g}$ Cys-scTNF, stained with Comassie. (c) Cytotoxicity assay on $\mathrm{Kym}-1$ cells of Cys-scTNF and TNF $(0-10 \mathrm{ng} / \mathrm{ml})$.

\subsubsection{Coupling of Cys-scTNF to Amino-Functionalized Nanoparticles}

In a first step amino-functionalized polystyrene nanoparticles (NSL-PMI) were activated with the chemical crosslinker sulfo-SMCC (Figure 3-27). Next, Cys-scTNF or L-cysteine was incubated with activated particles for $1 \mathrm{~h}$ at room temperature. The cysteine residue at the $\mathrm{N}$ terminal extension of Cys-scTNF is capable to form a C-S-thioether with the maleimide groups on the particle surface resulting in scTNF-nanoparticles. This oriented presentation of Cys-scTNF at the surface of the particles permits binding and activation of TNF receptors. 


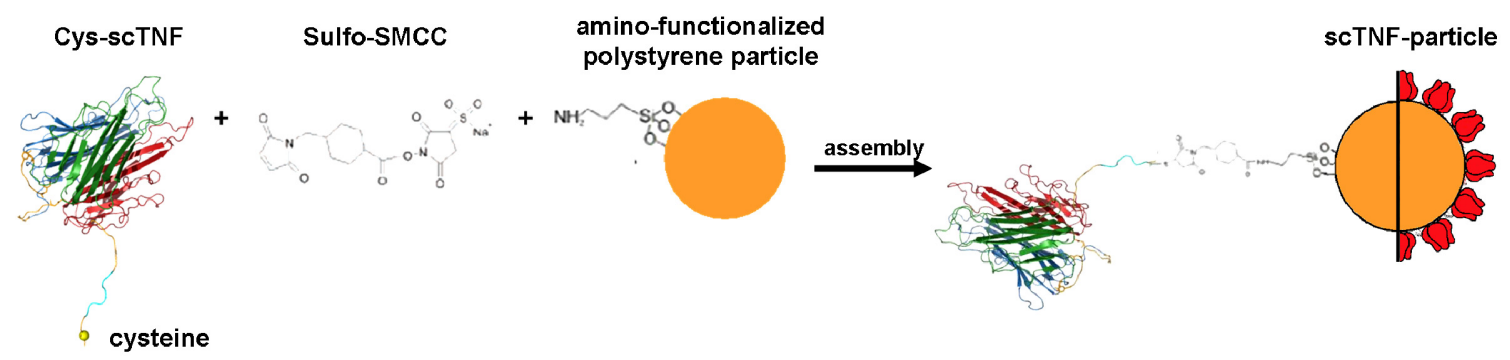

Figure 3-27: Coupling scheme of scTNF-Nanoparticles. Amino-functionalized green-fluorescing particles (NSL-PMI) are activated using the heterobifunctional crosslinker sulfo-SMCC which is coupled to the amino groups of the particles. The maleimide group of the crosslinker reacts with the free sulfhydryl group of the Cys-scTNF molecule. Calculated size: sulfo-SMCC: $0.13 \mathrm{~nm}$, Cys-scTNF: $5 \mathrm{~nm}$, particle: $186 \mathrm{~nm}$ )

The bioactivity of the scTNF-particles was determined in cytotoxicity assays with the TNFsensitive cell line Kym-1. Activated amino-functionalized particles incubated with L-cysteine showed no cytotoxic effect up to a concentration of $50 \mu \mathrm{g} \mathrm{P} / \mathrm{ml}$ (data not shown). A dose dependent response in bioactivity of the scTNF-particles was observed when varying amounts of Cys-scTNF were used for coupling to amino-functionalized and activated particles. The maximal cytotoxicity on Kym-1 cells was reached at a range of 10-30 $\mu \mathrm{g}$ CysscTNF per mg particle (Figure 3-28, Martin Altvater, diploma thesis, 2009). For all further studies scTNF-P were prepared by coupling $30 \mu \mathrm{g}$ Cys-scTNF per mg particles. The amount of coupled Cys-scTNF was determined by an indirect TNF-ELISA. The coupling efficiency was calculated to be $2.7 \pm 0.5 \mu \mathrm{g}$ Cys-scTNF/mg P. This corresponds to approximately 40 coupled scTNF molecules per particle (Martin Altvater, diploma thesis, 2009).

a)

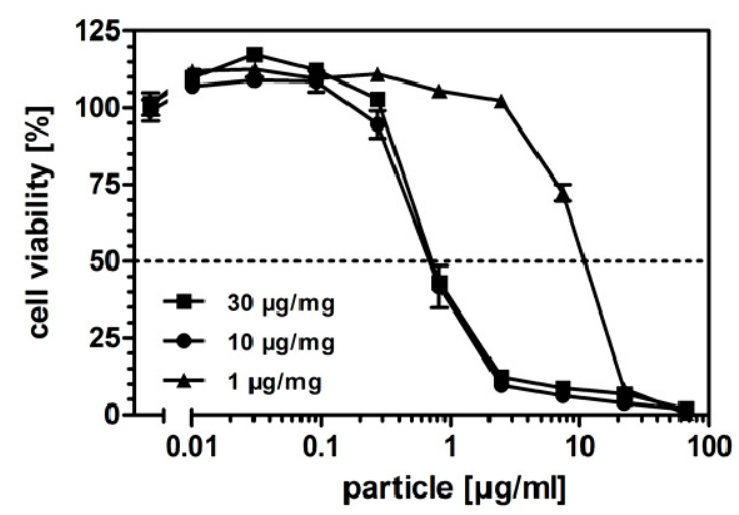

b)

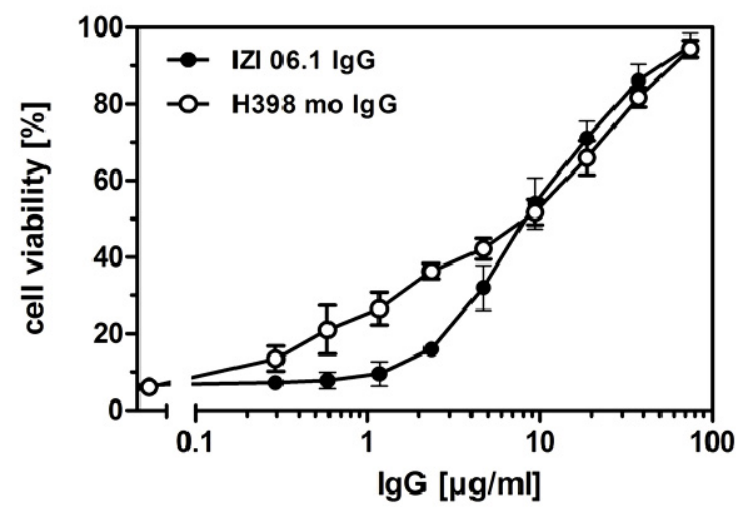

Figure 3-28: Bioactivity of scTNF-functionalized particles. (a) Cytotoxicity on Kym-1 cells (15.000 cells/well) of scTNF prepared by coupling different amounts of Cys-scTNF to sulfo-SMCC activated amino-functionalized particles (1-30 $\mu \mathrm{g}$ Cys-scTNF per mg P; carried out by Martin Altvater). After 16 $\mathrm{h}$ cells were analyzed by crystal violet assay. (b) Inhibition of scTNF-P mediated cytotoxicity. Kym-1 cells $(15.000$ cells/well) were preincubated for 40 min with IZI $06.1 \mathrm{lgG}$ or H398 mo IgG $(0-75 \mu \mathrm{gg} / \mathrm{ml})$. Constant amount of $1 \mu \mathrm{g} \mathrm{P} /$ well scTNF-P was applied. After $8 \mathrm{~h}$ cells were analyzed by crystal violet assay. $100 \%$ viability $=$ untreated control (only medium). 
In order to confirm that induced cytotoxicity on Kym-1 cells is due to binding of scTNFparticles to TNF-receptors on the cell surface, a blocking assay was performed. Therefor Kym-1 cells were preincubated with a humanized IgG (IZI 06.1 lgG) or a murine lgG (H398 mo $\lg G)$, respectively. These $\lg G$ s bind with high affinity to the cysteine-rich domains 1 and 2 of TNF-receptor 1 and compete for ligand binding (Kontermann et al., 2008). XY shows efficient and concentration-dependent blockage of scTNF-P-mediated cytotoxic action on $\mathrm{Kym}-1$ cells by both IgGs with IC50 values of approximately $10 \mu \mathrm{g} / \mathrm{ml}$.

\subsubsection{Cys-scTNF-functionalized TLP}

To generate scTNF-TLP lipid-coating was performed by the sonification method (3.2.1.2). The lipid mixture was composed of EPC, cholesterol and $\mathrm{mPEG}_{2000}$-DSPE (2 mol\%). Targeted lipid-coated scTNF-functionalized nanoparticles (scTNF-TLP) were prepared by coupling anti-FAP scFv LCH3 to Mal-PEG 2000 -DSPE micelles and following insertion into scTNF-LP (5 mol\% micellar lipid in respect to LP lipid) for $30 \mathrm{~min}$ at $55^{\circ} \mathrm{C}$. Non-coupled scFv molecules were subsequently removed by centrifugation. The size determined by dynamic light scattering intensity of scTNF-LP and scTNF-TLP is presented in Table 6. The diameter of amino-functionalized particles increased after coupling of scTNF by $16 \mathrm{~nm}$, which correspond to the size of one scTNF molecule. A decrease in size of $9 \mathrm{~nm}$ was measured after lipid coating. A further reduction by $12 \mathrm{~nm}$ was measured after insertion of scFv-coupled micelles, probably due the amount of inserted PEG-chains.

Table 6: Characterization of ScTNF-functionalized nanoparticles $P$, particles; scTNF-P, scTNF-functionalized particles; scTNF-LP, lipid-coated scTNF-functionalized particles; scTNF-TLP, targeted lipid-coated scTNF-functionalized particles

\begin{tabular}{lcccc}
\hline Formulation & $\begin{array}{c}\text { mPEG-DSPE } \\
\text { (mol\%) }\end{array}$ & $\begin{array}{c}\text { scFv-Mal-PEG-DSPE } \\
\text { (mol\%) }\end{array}$ & $\begin{array}{c}\text { Size } \\
\mathbf{( n m})\end{array}$ & PDI \\
\hline scTNF-functionalized particles based on NSL-PMI & & \\
P & - & - & $186 \pm 1.4$ & 0.11 \\
scTNF-P & - & - & $202 \pm 1.8$ & 0.14 \\
scTNF-LP & 5 & - & $193 \pm 2.2$ & 0.05 \\
scTNF-TLP & 5 & 5 & $190 \pm 0.8$ & 0.10
\end{tabular}

\subsubsection{Binding Studies of anti-FAP scTNF-TLP}

The scTNF-TLP preparations were analyzed for binding to FAP-expressing (HT1080 FAP $\left.{ }_{\text {hu }}\right)$ and FAP-negative (HT1080 wt) cells. Binding of scTNF-particles, scTNF-LP and scTNF-TLP was detected through a green fluorescence dye (perylene monoimide, PMI) incorporated into 
polystyrene particles. Uncoated scTNF-particles showed a strong nonspecific binding to FAP-positive as well as to FAP-negative cells (Figure 3-29). Flow cytometry analysis of scTNF-LP confirmed that lipid coating reduces nonspecific binding to these cell lines. As expected the anti-FAP scTNF-TLP exhibited a specific binding to HT1080 FAP $_{\text {hu }}$ cells, while no binding to HT1080 wild-type cells was observed.

scTNF-P

HT1080 wt
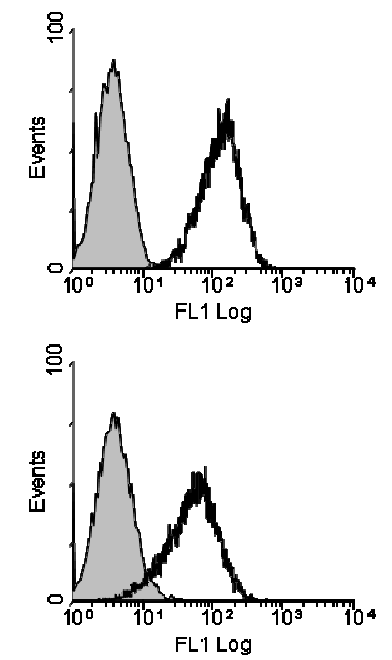

ScTNF-LP
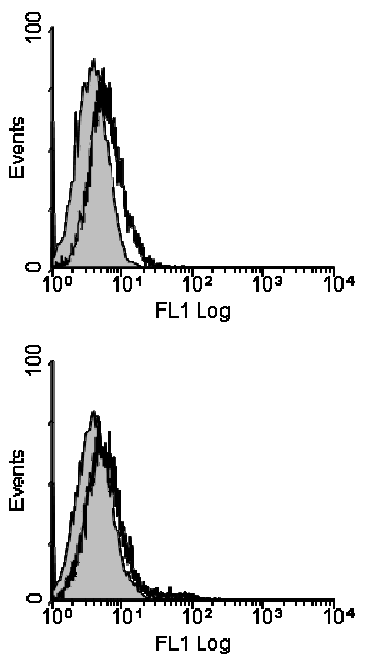

SCTNF-TLP
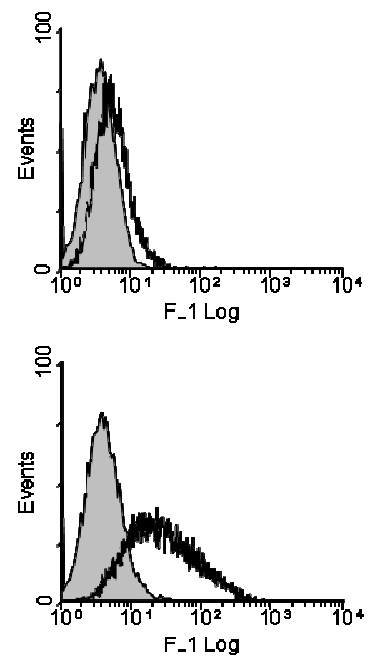

Figure 3-29: Flow cytometry analysis of scTNF-, scTNF-LP and anti-FAP scTNF-TLP. ScTNF-LP and scTNF-TLP were prepared with 5 mol\% $\mathrm{mPEG}_{2000}$-DSPE and insertion of $5 \mathrm{~mol} \%$ micellar scFvcoupled Mal-PEG ${ }_{2000}$-DSPE lipid. Grey fill: cells alone, black line: cells incubated with particle preparations at $3 \mu \mathrm{g} / 250000$ cells.

\subsubsection{Internalization Studies of anti-FAP scTNF-TLP by Confocal Microscopy}

Additionally to flow cytometry analysis internalization studies by confocal microscopy were performed. In order to investigate the internalization of anti-FAP scTNF-TLP sections through the z-axes were analyzed. Therefor HT1080 cells were incubated with anti-FAP scTNF-TLP prepared from scTNF-functionalized, red-fluorescing particles (NSL-Lum). The cells were incubated under permanent rolling in culture medium for $6 \mathrm{~h}$ at $37{ }^{\circ} \mathrm{C}$ followed by a cell boundary staining. Confocal microscopy revealed a strong internalization of anti-FAP scTNFTLP into FAP-expressing cells that could be clearly seen in the vertical and horizontal sections (Figure 3-30). 


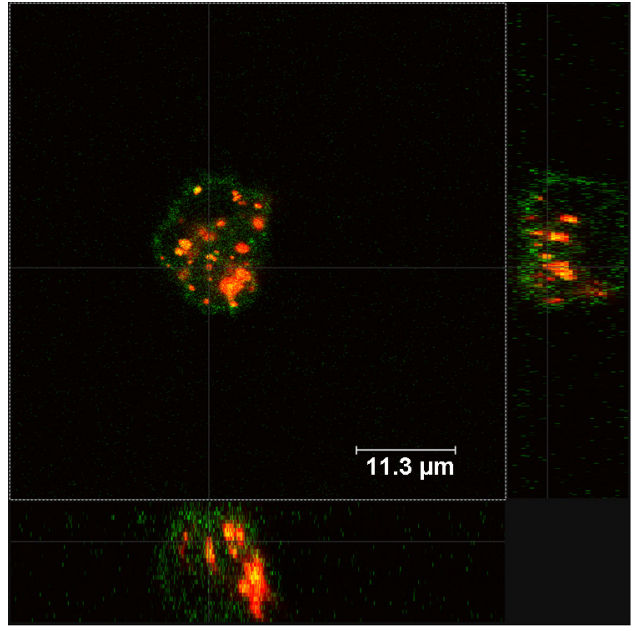

Figure 3-30: Internalization study of anti-FAP scTNF-TLP. Confocal microscopy image of a $\mathrm{HT} 1080 \mathrm{FAP}_{\text {hu }}$ cell incubated with scTNF-TLP for $6 \mathrm{~h}$ at $37^{\circ} \mathrm{C}$. Sections through the z-axes clearly show internalization of red flourescing particles. Cell membrane was visualized by counter-staining with an anti-CD105 antibody (green).

\subsubsection{Cytotoxicity Assays on Kym-1 cells}

On the basis of the PEG-lipid shell of the scTNF-particles the bioactivity should be markedly reduced. Therefor cytotoxicity assays were performed. Lipid-coating of scTNF-functionalized nanoparticles strongly reduced cytotoxicity on target antigen-negative Kym-1 cells (Figure 3-31), thus demonstrating shielding of TNF action. The $I C_{50}$ values are presented in Table 7. After $24 \mathrm{~h}$ of incubation with the scTNF-particle preparation, the $\mathrm{IC}_{50}$ values were increased 13 fold for scTNF-LP $\left(\mathrm{IC}_{50}=2.7 \mu \mathrm{g} / \mathrm{ml}\right)$ and 33-fold for scTNF-TLP $\left(\mathrm{IC}_{50}=6.7 \mu \mathrm{g} / \mathrm{ml}\right)$, compared to scTNF-P $\left(\mathrm{IC}_{50}=0.2 \mu \mathrm{g} / \mathrm{ml}\right)$.

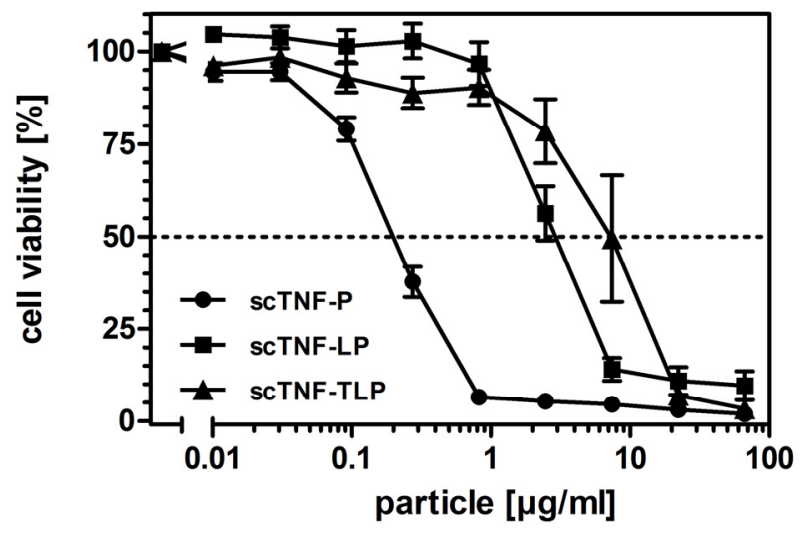

Figure 3-31: Lipid-coating reduces cytotoxicity activity. Kym-1 cells were incubated with varying amounts of scTNF-P, scTNF-LP and anti-FAP scTNF-TLP. After 24 hours cells were analyzed with crystal violet assay. 
Table 7: $\mathrm{IC}_{50}$ values of scTNF particles (in $\mu \mathrm{g} / \mathrm{ml}$ )

\begin{tabular}{lccc}
\hline Formulation & Kym-1 & HT1080 wt & HT1080 FAP $_{\text {hu }}$ \\
\hline scTNF-P & 0.2 & 0.8 & 0.6 \\
scTNF-LP & 2.7 & 18 & 13 \\
scTNF-TLP & 6.7 & $\sim 20$ & 3.3
\end{tabular}

\subsubsection{Cytotoxicity Assays on HT1080 cells}

For determination the bioactivity preparations on HT1080 cell lines, the scTNF-particle preparations were incubated with $\mathrm{HT}_{1080} \mathrm{FAP}_{\text {hu }}$ and HT1080 wild type cells under constant rolling in cell culture medium for $2 \mathrm{~h}$ at $37^{\circ} \mathrm{C}$. The particles were subsequently separated by a centrifugation step and cells were incubated for 16 more hours. The assays on HT1080 wildtype cells (Figure 3-32 a, Table 7) revealed an approximately 22-fold reduced toxicity for scTNF-LP $\left(\mathrm{IC}_{50}=18 \mu \mathrm{g} / \mathrm{ml}\right)$ compared to scTNF-P $\left(\mathrm{IC}_{50}=0.8 \mu \mathrm{g} / \mathrm{ml}\right)$. In case of scTNF-TLP $\left(\mathrm{IC}_{50} \sim 20 \mu \mathrm{g} / \mathrm{ml}\right)$ even a 25 -fold reduction could be shown. Therefore, lipid-coating of scTNFfunctionalized nanoparticles efficiently shielded from TNF action on wild-type cells. On FAPpositive cells scTNF-LP $\left(\mathrm{IC}_{50}=13 \mu \mathrm{g} / \mathrm{ml}\right)$ exhibited again a 22-fold reduction in cytotoxicity as compared to bare scTNF-P $\left(\mathrm{IC}_{50}=0.6 \mu \mathrm{g} / \mathrm{ml}\right)$. Importantly, scTNF-TLP $\left(\mathrm{IC}_{50}=3.3 \mu \mathrm{g} / \mathrm{ml}\right)$ showed a significant 4-fold higher cytotoxic activity on FAP-expressing cells (Figure 3-32 b, Table 7) than scTNF-LP $\left(\mathrm{IC}_{50}=13 \mu \mathrm{g} / \mathrm{ml}\right)$. These data suggest on the one hand for an effective shielding of TNF and on the other hand for an antigen-dependent uncoating process associated with a selective cytotoxicity towards FAP-expressing cells.

a)

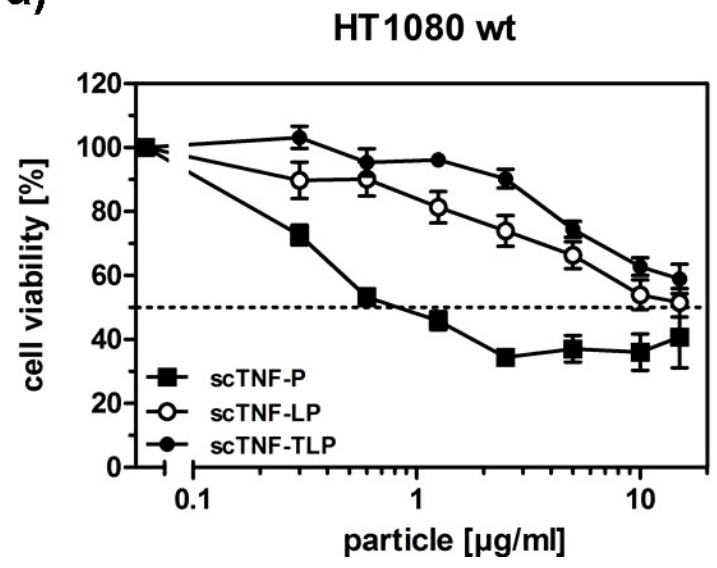

b)

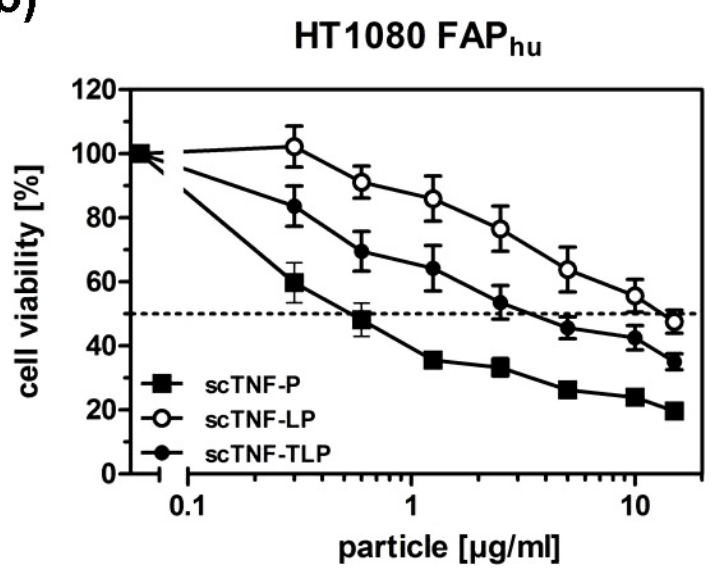

Figure 3-32: Cytotoxicity of scTNF-P, scTNF-LP and anti-FAP scTNF-TLP toward HT1080 cell lines. Cells were incubated for $2 \mathrm{~h}$ with varying amounts of scTNF-P, scTNF-LP and anti-FAP scTNFTLP at $37^{\circ} \mathrm{C}$. After separation of scTNF-particle preparations, cells were seeded in a 96-well plate and incubated o/n at $37^{\circ} \mathrm{C}$. After 16 hours cells were analyzed with crystal violet assay. (a) HT1080 wild type cells (b) HT1080 FAP ${ }_{\text {hu }}$ cells. 


\section{Discussion}

Polymeric nanoparticles displaying tumor necrosis factor on their surface are useful carrier systems capable of mimicking the bioactivity of membrane-bound TNF (Bryde et al., 2005). However, their in vivo applications are hampered by the two sided TNF action, especially the potential systemic action of TNF, which can lead to severe side effects and even to death. In the present study the implementation of targeted lipid-coated TNF-functionalized nanoparticle (scTNF-TLP) was achieved which may be a promising formulation of TNF that should enable a systemic application and induction of tumor selective activity.

In the first part of this study, novel scFv format were designed for the generation of targeted carrier system by site-directed coupling. As target antigen the fibroblast activation protein (FAP) was used exemplary. In the second part, two different methods for the coating of polystyrene-based nanoparticles with a sterically stabilized PEG-lipid shell were established.

By insertion of a newly designed scFv' format against FAP into the lipid coat, also a selective delivery of the embedded nanoparticle to antigen-positive target cells could be obtained. In the last part the different lipid-coating methods were applied for single-chain TNFfunctionalized nanoparticles resulting in a strong reduction of the cytotoxic effect on targetnegative cells. Importantly, the implementation of a targeting moiety mediated a selective delivery of the embedded TNF-functionalized nanoparticle to antigen-positive target cells.

\subsection{Novel scFv' Formats for the Generation of Targeted Carrier Systems}

A well defined method for scFv' molecules suitable for a site-directed coupling is the introduction of an additional C-terminal cysteine residue by genetic modifications (Marty et al., 2005; Völkel et al., 2004; Marty et al., 2002; Nielsen et al., 2002). Different cloning strategies based on a scFv molecule (scFv' 36) were persecuted. ScFv' 36 binds to the murine fibroblast activation protein (FAP) and is cross-reacting with human FAP. It contains one additional cysteine residue at the C-terminus of short length suitable for the generation of immunoliposomes (Baum et al., 2007). The first strategy focused on the insertion of one or more additional cysteine residues at the end of a C-terminal extension of varying length $(\mathrm{HC})$. This format results in a molecule with an extended spacing between the antibody construct and the reactive partner. The second strategy focused on the introduction of one additional cysteine residue within the flexible peptide linker ( $\mathrm{LC}, \mathrm{LCH})$, whereby no additional $\mathrm{C}$-terminal extension is required. 


\subsubsection{HC and LC Variants}

All scFv' variants could be expressed in E. coli and purified in a soluble form. Moreover, yields were similar to those of unmodified scFv 36 and scFv' 36 molecules, indicating that cysteine residues introduced at the C-terminal extension or within the peptide linker do not influence the expression as observed for other scFv' formats (Schmiedl et al., 2000). Under non-reducing conditions, SDS-PAGE and immunoblotting analysis revealed molecules of higher molecular weight which correspond to scFv dimers. Therefore it can be concluded that the cysteine residues are accessible and functional for the formation of disulfide-linked homodimers and are suitable for the coupling to reactive groups, e.g. of lipids. All six scFv' derivatives exhibited a similar binding activity towards target cells independent from the number and location of introduced cysteine residues. Additionally all fragments retained selectivity towards FAP.

For the coupling of the scFv' variants to liposomal carrier systems type II immunoliposomes were chosen. It is well known that PEGylated liposomes exhibit a longer blood circulation time. Furthermore a significantly reduced accumulation of PEGylated liposomes in the liver and spleen could be observed (Papahadjopoulos et al., 1991). However, using the conventional coupling method, only a low coupling efficiency between 10-20 \% could be observed for this type of immunoliposome (Baum et al., 2007; Völkel et al., 2004). For this reason the post-insertion method was used (Ishida et al., 1999). By this technique the scFv' molecules are coupled to preformed micelles composed of lipid with a reactive group. In a next step the scFv-micelles are inserted into liposomes. Thereby an exchange between lipids of the outer layer of liposomes and the scFv-coupled lipids takes place, normally at temperatures in the range of $40-60^{\circ} \mathrm{C}$. The optimal temperature depends on the transition temperature $\left(T_{m}\right)$, which is, despite of other factors, dependent on the composition of the liposomal bilayer. For instance, $\mathrm{T}_{\mathrm{m}}$ of hydrogenated soy PC was reported to be $54^{\circ} \mathrm{C}$ (Park et al., 2002). In general, the higher the temperature the more fluid is in the liposomal membrane and it can therefore be concluded that insertion efficiency of scFv-coupled micelles increases with temperature. However, enhancement of insertion temperature is limited by the thermal stability of the used scFv' molecule. The melting point for all HC and LC constructs was determined to be around $56-59^{\circ} \mathrm{C}$. Therefore the post-insertion could be performed at $55^{\circ} \mathrm{C}$, a temperature which resulted in optimal insertion efficiencies as established in a former diploma thesis (Anke Kolbe, 2007). ScFv' molecules with different specificities can differ significantly in their thermal stability. For instance, a scFv against carcinoembryonic antigen (CEA) possesses a melting point of about $40^{\circ} \mathrm{C}$. As a consequence the post-insertion step had to be performed at lower temperatures, and longer incubation times were required to achieve strong binding of these immunoliposomes to CEA-expressing cells (Rüger, 2008). 
All scFv' molecules could be coupled to maleimide-containing lipids. Coupling efficiency was similar for all scFv' derivatives containing one additional cysteine residue irrespective of whether it is inserted at C-terminus or within the linker sequence, and was determined to be in the range of $80-90 \%$. This is comparable to other antibody fragments with one additional cysteine residue at the C-terminus (Park et al., 2001). For the corresponding immunoliposomes also similar binding activities could be observed. Flow cytometry analyses revealed an increase in binding of immunoliposomes to murine FAP expressing cells with an amount of $2 \mathrm{~mol} \%$ added scFv-micelles compared to the insertion of $0.6 \mathrm{~mol} \%$ Mal-PEGcoupled scFv molecules. It was reported for anti-HER2 Fab-immunoliposomes that increased antibody fragment density correlates with an increased binding and internalization, reaching a plateau of approximately 40 Fab' molecules per liposome (Park et al., 2001). It can therefore be concluded that the optimal micelle to total lipid ratio is about $2 \mathrm{~mol} \%$ in order to achieve best binding activity for the immunoliposomes generated from these different scFv' 36 variants. Immunoliposomes generated from the scFv' construct comprising three additional cysteine residues at the C-terminal extension (HC3) demonstrated a reduced binding activity although the scFv' construct exhibited a coupling efficiency of about $98 \%$. SDS-PAGE analysis revealed the coupling of three Mal-PEG ${ }_{2000}-\mathrm{DSPE}$ molecules. Probably the insertion into the lipid bilayer may lead to a sterically interference, leading to an unfavorable orientation of the scFv molecule on the liposomal surface and therefore resulting in a reduced accessibility and cell binding. Also with this scFv variant the best binding activity could be seen by insertion of 2 mol\% Mal-PEG-micelles with respect to total lipid.

The immunoliposomes described in literature so far were prepared from scFv' molecules that contain one or more additional cysteine residues at the C-terminus (Cheng et al., 2007; Völkel et al., 2004; Mamot et al., 2003; Marty et al., 2001) or that have been conjugated through other reactive coupling reagents, like amino groups (Hu et al., 2006; Gosk et al., 2005). The conjugation by amino-reactive groups bears the risk of an undirected and nonorientated coupling and might influence the antigen-binding activity. In contrast engineered insertion of cysteine residues ensures a site-directed as well as oriented coupling. It is known that the C-terminal extension is located opposite to the antigen binding site therefore coupling to reactive groups of e.g. PEG chains should not interfere with the recognition of the target cells. This was further confirmed in this study applying three scFv constructs with Cterminal cysteines. Interestingly, functional immunoliposomes could also be generated with scFv' molecules (LC) which contain the additional SH-group within the linker. As mentioned above three positions (1-3) within the linker peptide were compared. No differences in coupling efficiency and binding to target cells was detectable between the LC constructs among each other or in comparison to the variants possessing one cysteine at the $\mathrm{C}$ - 
terminus. Structural models of FAP may elucidate these data: the three positions of the cysteine residues are located at the bottom of the scFv molecule and therefore opposite to the antigen binding site. In the literature, similar scFv' fragments which possess an additional cysteine residue within the linker have been described (Shen et al., 2008; Albrecht et al., 2006; Shen et al., 2005). For instance, the scFv A10B molecule possessing an additional cysteine at position one of a 15 aa long peptide linker was applied for the generation of highly sensitive and specific scFv immunosensors (Shen et al., 2008). In this study the suitability of additionally cysteines introduced at different position within the flexible peptide linker of scFv' molecules for the generation of immunoliposomes, but also other application were confirmed. This variants are also suitable for site-directed PEGylation to improve the pharmacokinetic properties, the generation of targeted gold nanoparticles or immunosensors (Natarajan et al., 2005; Backmann et al., 2005; Ackerson et al., 2006).

\subsubsection{LCH Variants}

This approach was further developed by applying scFv' molecules with the additional cysteine residue and the hexahistidyl-tag (His-tag) present in the linker sequence. This novel scFv format has the advantage that except for the amino acids of the linker sequence no further amino acid residues are required. In this way, the scFv' molecules are reduced to a minimal length and composition that is required for an easy one-step purification via IMAC and site-directed coupling to reactive molecules. Both $\mathrm{LCH}$ variants $(\mathrm{LCH} 1 / 3)$ presented in this study could be produced by bacteria in a soluble form and could be purified to a sufficient purity from periplasm in a coupling-active form, indicated by the formation of dimers. The determined melting points were around $56-58^{\circ} \mathrm{C}$, thus suitability for the postinsertion method is given. Both scFv' 36 molecules showed in FACS analysis a weak binding signal to target cells probably due to a detection difficulty. The binding is determined by detecting the scFv' molecules with an antibody directed against the His-tag, thus it is likely that the recognition of the His-tag within the linker sequence is impaired due to the structure of the molecule. Nevertheless immunoliposomes generated by these scFv' format exhibited a strong binding to target cells comparable to those prepared with LC variants. On the one hand this is evident from the binding ability for both LCH molecules to the antigen FAP and on the other hand the suitability for site-directed coupling. Internalization into target cells and subsequent distribution of drug-loaded immunoliposomes was found to be major prerequisites for efficient anti-tumoral activity (Kirpotin et al., 2006). The most important ways for uptake are the clathrin-mediated endocytosis, caveolae-mediated endocytosis and the socalled non-clathrin-non-caveolae-mediated endocytosis. But also phagocytosis, macropinocytosis and membrane fusion are conceivable (Duve et al., 1975). Here, the 
internalization of anti-FAP scFv LCH3 immunoliposomes by FAP-expressing cells could be shown. A membrane fusion of the liposomes can be most likely ruled out since the used membrane marker DiO is strongly accumulated within the cells. Similarly unspecific uptake by macropinocytosis can be excluded because no uptake by wild type cells could be observed. Colocalization studies of other anti-FAP scFv immunoliposomes with endosomal marker protein fused to CFP revealed that the liposomes were internalized into the endosomal compartment of the cells (Baum et al., 2007), which was also shown for antiEGFR immunoliposomes (Mamot et al., 2003). These data indicate that the immunoliposomes are taken up as a whole via endocytosis. To ensure these data internalization studies using endocytosis inhibitors that prevent uptake of liposomes by inhibiting certain mechanism of internalization can be performed. For instance, the clathrinmediated endocytosis can be prevented by the usage of chlorpromazine (Wang et al., 1993) or methyl-ß-cyclodextrin (Rodal et al., 1999). The latter also prevent caveolae-mediated endocytosis which can be directly inhibited also by filipin (Sieczkarski \& Whittaker, 2002). In general, the internalization of anti-FAP immunoliposomes is insofar surprising, as FAP is not a cell surface receptor but a proteinase hypothesized to be involved in degradation and remodeling of the extracellular matrix, e.g. of tumor tissue (Park et al., 1999). Recent studies pointed out that FAP executes its biological function through a combination of the protease activity and the ability to form complexes with other membrane-bound signaling molecules (Kelly, 2005). An involvement of this function at the internalization process of anti-FAP immunoliposomes is rather unclear.

For implementing further approaches for detection and purification other short purification and/or detection tags than the His-tag may be integrated into the linker sequence (Terpe, 2003). Furthermore one can envisage to abandon the affinity tags since anti-CD19 scFv immunoliposomes showed a rapid clearance by an increased liver uptake probably due to the His- and/or myc-tags (Cheng \& Allen, 2008). Additionally, the Food and Drug Administration (FDA) does not accept the utilization of these kinds of tags. Either one excludes these tags or replaces them by antibody-tags, e.g. a FLAG-tag. Alternatively, single-step purification for some scFv fragments using protein $A$ and $L$ has already been successfully applied (Das et al., 2005; Nellis et al., 2005).

In summary, all newly designed scFv constructs extended the application for the generation of targeted nanoparticulate carrier systems, especially the novel designed scFv' LCH format. A cleavage of the additionally cysteine residue within the linker sequence is unlikely compared to cysteine residues introduced at the C-terminal extension. Thus, the occurrence of coupling inactive molecules is reduced. Furthermore, the number of additional amino-acid 
residues in the antibody molecules is limited. Moreover, the SH-group is located opposite to the antigen-binding site, as shown by structural analysis and should therefore be highly accessible for site-directed coupling without interference with the binding ability. These properties predestinate the LCH variants as targeting moieties for nanoparticulate carrier systems. In the following, the scFv'36 LCH3 format was used for the generation of targeted lipid-coated composite nanoparticles.

\subsection{Targeted Composite Nanoparticles}

The second part of the present study focused on the conversion of non-functionalized, amino- and cytokine-functionalized particles into targeted multicomposite nanoparticles. This was achieved by two steps. In the first step particles were coated with a mixture of egg phosphatidylcholine (EPC), cholesterol (Chol) and $\mathrm{mPEG}_{2000}-\mathrm{DSPE}$ resulting in a shielding of the particle surface (lipid-coated particles, LP) and a reduction of non-specific binding. In a second step an antibody fragment (scFv) coupled to micellar Mal-PEG ${ }_{2000}$-DSPE was inserted into the lipid-coat, resulting in targeted lipid-coated particles (TLP).

\subsubsection{Generation of Lipid-Coated Particles}

In the present study two techniques have been established for the coating of particles. By the first one a particle/lipid mixture is extruded at an elevated temperature, whereby at the second method a particle/liposome mixture is sonificated. After preparation of lipid-coated particles (LP) a sucrose gradient centrifugation was performed. This technique is often used for the purification of fluorescent-loaded liposomes (Haginoya et al., 2005). Hereby the free liposomes and uncoated particles could be efficiently separated from LP, indicating that the LP possesses physiochemical properties different from empty liposomes and particles, e.g. possessing a density in-between. The lipid-coating was further confirmed by the incubation with the nonionic detergent Triton X100 that interacts with the lipid coat and leads to their destruction indicated by appearance of lipids and free particles. In the course of experiments it became apparent that mostly all particles were lipid-coated. Therefore it was decided to use a single centrifugation step to separate only the empty liposomes instead of the purification by density centrifugation. In the literature, various particles for the generation of core-shell system have been described, e.g silica particles, biocompatible gold nanoparticles or biodegradable polylactide-co-glycoide (PLGA) nanoparticles (Filchtinski et al., 2008; Li et al., 2009; Chan et al., 2009). Cryo-EM studies revealed a lipid-bilayer for encapsulated silica particles (Mornet et al., 2005), whereas for core-shell nanoparticles composed of a PLGA core a hydrophilic PEG shell and a lecithin monolayer at the interface of the hydrophobic 
core was shown (Chan et al., 2009). Another approach is the direct PEGylation of the particle. Hereby the PEG polymers were added during the process of formation of the core resulting in PEG-functionalized nanoparticles. This was exemplary shown for a PLGA-blockPEG copolymer. The usage of a carboxy-functionalized PEG chain enabled the coupling of targeting molecules (Farokhzad et al., 2006). The formation of a bilayer on polystyrene particles was shown to depend on the one hand on the amphiphile type and concentration of the particle and on the other hand on the kind and amount of functional groups on polystyrene particle surface (Carmona-Ribeiro \& Lessa, 1999). For neutral phospholipids a deposition on amidine polystyrene particles as a monolayer was shown, whereas cationic liposomes and oppositely charged particles resulted in the formation of a bilayer (Tsuruta et al., 1997). It is postulated that several attractions like electrostatic and/or hydrophobic ones led to an interaction of a liposome and a polystyrene particle. This close proximity may disrupt the lipid-bilayer and promote an adsorption of a bilayer on the particle surface. However, by further interaction of the bilayer with other polystyrene particles the bilayer structure may completely be destroyed, whereby monolayer coverage on each particle can be generated (Carmona-Ribeiro \& Lessa, 1999).

In the present study it was not yet possible to visualize the lipid coat, e.g. by cryo-TEM. However, the measured increase in size of LP can be taken as evidence for coating of the particles with lipids. Interestingly, the increase in size was dependent on the amount of $\mathrm{mPEG}_{2000}$-DSPE. LP containing 2 mol\% $\mathrm{mPEG}_{2000}$-DSPE possessed a much larger hydrodynamic radius than LP containing $5 \mathrm{~mol}^{2} \mathrm{mPEG}_{2000}-\mathrm{DSPE}$, independent whether non-functionalized or amino-functionalized particles were covered. Additional, flow cytometry analyses using red fluorescing particles and lipids containing $\mathrm{DiO}$ as green fluorescing lipid label further confirmed lipid-coating of all particles. Compared to non-coated particles which showed a distinct population of red fluorescing particles, lipid-coated particles demonstrated a scattered distribution of red and green fluorescent signals. This is in accordance with the PDI and indicates the decoration of particles with varying amounts of lipids. The observed reduction of red fluorescence for the lipid-coated particles is likely caused by quenching effects of the lipid coat. The presence of empty liposomes can be ruled out since they can not be visualized by flow cytometry alone. The reduced unspecific binding of LP compared to noncoated particles to FAP-negative as well as to target cells as can be taken as another evidence for the lipid coverage of particles. But at this stage, it is not possible to discriminate between particles covered with a lipid bilayer and those composed of multiple bilayers or a monolayer. Preliminary cryo-TEM studies did not provide information about the state of the lipid coat. However, the coverage of lipid-coated PLGA nanoparticles was characterized using atomic force microscopy (AFM) investigations (Schäfer et al., 2008), which could be a 
useful technique for further studying of the lipid coverage of polystyrene particles.

\subsubsection{Generation of Targeted Lipid-Coated Particles}

By means of the successful lipid-coating of polystyrene particles an important basis was established for the further implementation of an active targeting. This was reached by insertion of the newly designed scFv' format LCH3 against FAP into the lipid coat employing the post-insertion method. The resulting targeted lipid-coated particles (TLP) showed a strong binding activity only to target cells, independent from the kind of used particles (nonfunctionalized or amino-functionalized particles). An increase in binding for TLP to FAPexpressing cells could be observed while a higher amount of micellar Mal-PEG-DSPE was inserted, accompanied with a decrease in unspecific binding. This indicates that Mal-PEGDSPE contributes to reduction of nonspecific binding and furthermore functional TLP were formed although the amount of PEG is very high. In the literature an upper limit of 15-20 mol\% for the insertion of PEG molecules into lipid bilayers has been described (Hristova et al., 1995), which can be associated with the generation of targeted mixed micelles that can compete for binding of TLP to target cells. Currently the existence of such mixed micelles cannot be excluded, although the used PEG concentrations are below the above mentioned limit. Furthermore, lipid-coated particles did not show an altered binding activity after insertion of additional $5 \mathrm{~mol} \%$ micellar lipid into the lipid coat ( $2 \mathrm{~mol} \% \mathrm{mPEG}-\mathrm{DSPE})$ as compared to those composed of 5 mol\% mPEG-DSPE. Quite the opposite, a slightly higher nonspecific binding to FAP-negative cells could be observed for LP and TLP covered with a lipid coat containing lower amount of mPEG-DSPE. Currently the scFv concentration of TLP is not yet known. In absolute terms the highest concentration of inserted scFv that could be expected would be approximately $10 \mu \mathrm{g} \mathrm{scFv}$ per mg particle. This concentration is below the detection rate of conventional protein assays. Furthermore, polystyrene particles demonstrated some interference with the applied protein assays. Therefore new detection methods have to be implemented for the determination of the ScFv amount of TLP. For example, a determination of the coupling efficiency using radio-labeled scFv molecules has bee described for anti-FAP immunoliposomes (Baum et al., 2007). An indirect determination using a recombinant fluorescence protein as described for iron oxide nanoparticles is also conceivable (Yang et al., 2009).

TLP were highly stable under physiological conditions with respect to antigen-binding activity after incubation for up to 24 hours in human plasma at $37^{\circ} \mathrm{C}$. Importantly, TLP retained selectivity towards antigen-expressing cells and demonstrated no increase in unspecific binding to FAP-negative cells, indicating stability of the protective lipid-coat as well as of the inserted antibody fragment. Internalization studies further confirmed the stability of the lipid 
coat in serum containing medium at $37^{\circ} \mathrm{C}$ for up to 6 hours. Only binding of anti-FAP TLP to target cells led to an efficient antigen-dependent internalization since no uptake was seen with FAP-negative cells. These data are highlighting the suitability of this lipid-coat and embedded targeting moiety for an application under physiological conditions.

Preliminary pharmacokinetic studies indicated an increased circulation time of lipid-coated particles compared to free particles after i.v. injection into mice, which is in accordance to experiments of uncoated polystyrene particles of a size of $60 \mathrm{~nm}$ and $250 \mathrm{~nm}$ revealing a rapid clearance from the blood by macrophages of the reticuloendothelial system (Moghimi, 1997). Lipid-coated silica particles displayed an 11-fold increase in circulation half-life time (van Schooneveld et al., 2008). However, the pharmacokinetic properties of the TLP are also determined by the targeted lipid coat which represents an anti-FAP immunoliposome. Although no data of pharmacokinetic properties for FAP targeted liposomes are available, there are some data for immunoliposomes endowed with other antibody fragments. For instance, anti-HER2 doxorubicin-loaded immunoliposomes showed a long circulation time in rats $\left(t_{1 / 2} 12-14 \mathrm{~h}\right.$ ) with no reduction after multiple injections (Park et al., 2002; Park et al., 2001). Similar pharmacokinetic profiles could be demonstrated with anti-CD19 stealth immunoliposomes loaded either with vincristine or doxorubicin (Sapra et al., 2004).

First in vivo studies using a PEARL ${ }^{\mathrm{TM}}$ Imager indicated a rapid accumulation within the liver for uncoated particles, whereas LP and TLP seemed to be more increased in the spleen and kidneys after a longer period of time as compared to uncovered particles. For lipid-coated silica particles an accumulation in the liver and negligible increase in spleen, heart and kidneys was demonstrated. Nevertheless, maximum accumulation was not reached 4 hours post injection as compared to bare silica particles which reached the maximum after one hour. In general uncoated silica particles always accumulated to a higher extent in liver and spleen than that observed for lipid-coated silica particles (van Schooneveld et al., 2008). PEGylated anti-EGFR magnetic iron oxide nanoparticles displayed a further reduction of accumulation in the liver and spleen combined with a long-circulation capacity (Yang et al., 2008). Despite of the promising data concerning the pharmacokinetic properties, further investigation has to be performed. Furthermore the long term physiochemical properties of the different particle preparations have to be considered, e.g. size and shape. For the latter a dominant role in phagocytosis in vivo has been described (Champion et al., 2009; Champion et al., 2006; Shanbhag et al., 1994).

\subsubsection{Cell Death Mediated by scTNF-Functionalized Nanoparticles}

Silica particles functionalized with a recombinant TNF derivative in an orientated way have been shown to possess a very high and specific bioactivity (Bryde et al., 2005). These 
particles raised a potent activation of both TNF receptors (TNFR1, TNFR2), thus mimicking action of the membrane-bound form of TNF (Grell et al., 1995). However, the physical natures of the particles and the applied TNF derivative have a few drawbacks. Besides covalent coupling some adsorption of the soluble TNF derivative was observed that was estimated to be $10 \%$ of the covalent coupled material. Furthermore, the particles showed some minor leakiness which can be explained by a slow release of the adsorbed TNF and/or by a reversible dissociation of TNF trimers that are covalently linked to particle surface by only one of its monomers (Bryde et al., 2005).

In this study amino-functionalized polystyrene particles were chosen as basis for coupling since no adsorption effects are known. Also, a distinct TNF format was used. It was obtained from a single-chain derivative (scTNF) composed of three TNF monomers linked to each other with increased stability and anti-tumor activity (Krippner-Heidenreich et al. 2008). To achieve site-directed coupling to the functionalized particle surface an additional cysteine residue was genetically introduced at the $\mathrm{N}$-terminal extension (Cys-scTNF). The modified TNF derivative showed a slightly increased bioactivity compared to soluble TNF on Kym-1 cells expressing elevated levels of TNFR1 and TNFR2. The orientated presentation of CysscTNF on the particle surface, coupled via the crosslinker sulfo-SMCC could be demonstrated by the effective induction of apoptosis in Kym-1 cells as well as in HT1080 cells. The latter express moderate levels of TNFR1, but to increase sensitivity towards receptor-mediated apoptosis a simultaneous down regulation of anti-apoptotic proteins by addition of the protein synthesis inhibitor cycloheximide $(\mathrm{CHX})$ is required.

The used amino-functionalized polystyrene particles demonstrated a dependence of cytotoxicity in relation to the Cys-scTNF input in the coupling reaction. A saturation in coupling could be found at 10-30 $\mu$ g Cys-scTNF input per mg particles. This corresponded to approximately $3 \mu \mathrm{g}$ Cys-scTNF per mg particles or to 40 Cys-scTNF molecules per particle. The coupling maximum for TNF-functionalized silica particles was determined as marginally higher. In average, $5 \mu \mathrm{g}$ TNF could be coupled per mg of particle independent of their size (Bryde et al., 2005). It was suggested that resulting particles contained a much higher TNF density on particle surface compared to a typical receptor density of a TNF-responsive cell. Therefore, coupled TNF should be sufficient for particle-mediated cell death. However, the $\mathrm{IC}_{50}$ value of scTNF-P $(0.2 \mu \mathrm{g} / \mathrm{ml})$ on $\mathrm{Kym}-1$ cells corresponds to a calculated concentration of approximately $0.5 \mathrm{ng} / \mathrm{ml}$ Cys-scTNF and is about fivefold higher than the typical $\mathrm{IC}_{50}$ of soluble Cys-scTNF on this cell line, suggesting that a part of the conjugated Cys-scTNF was either not accessible or was inactivated during the coupling process. This has to be analyzed in further studies. 


\subsubsection{Generation of Anti-FAP scTNF-Targeted Lipid-Coated Particles}

The schematic representation for the generation of targeted lipid-coated scTNFfunctionalized particles is presented in Figure 4-1. In brief, scTNF-P are generated by coupling Cys-scTNF to sulfo-SMCC-activated particles. Lipid-coated scTNF-P (scTNF-LP) are either produced by adding the scTNF-P to a lipid film and subsequent extrusion through a $200 \mathrm{~nm}$ membrane at $60{ }^{\circ} \mathrm{C}$ or by sonification combined with preformed stealth liposomes at $60{ }^{\circ} \mathrm{C}$. In parallel, the scFv' molecule is coupled through the sulfhydryl group of a genetically introduced cysteine residue to $M a l-P E G_{2000}-D S P E$ micelles and inserted into scTNF-LP by incubation for $30 \mathrm{~min}$ at $55{ }^{\circ} \mathrm{C}$ resulting in targeted lipid-coated scTNF-P (scTNF-TLP).

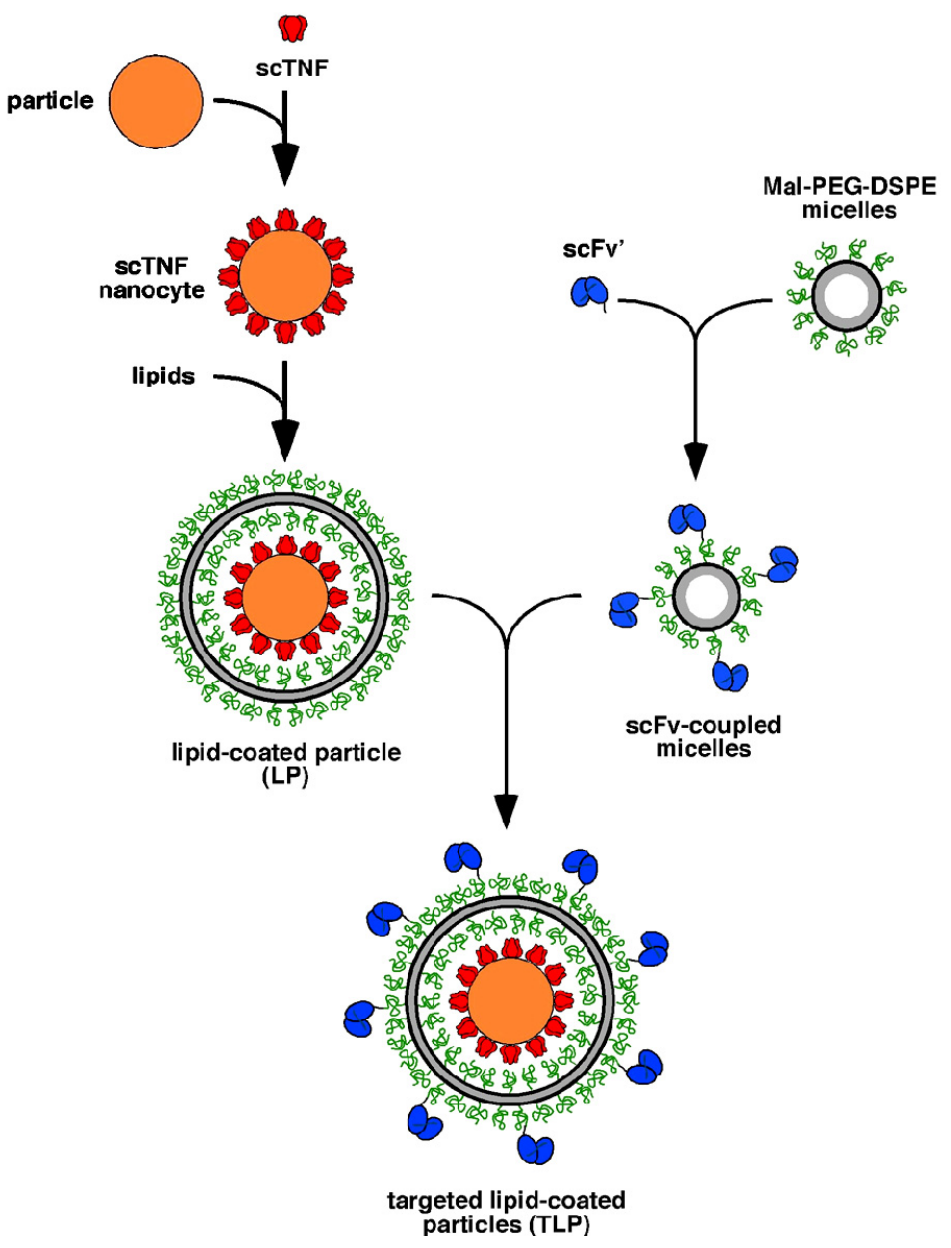

Figure 4-1: Strategy for the generation of targeted lipid-coated scTNF-particles (scTNF-TLP). ScTNF-functionalized particles (scTNF-P) are generated by coupling scTNF to sulfo-SMCC-activated nanoparticles. Lipid-coated scTNF-functionalized polystyrene particles (scTNF-LP) are either produced by adding scTNF-P to a lipid film and subsequent extrusion through a $200 \mathrm{~nm}$ membrane at $60{ }^{\circ} \mathrm{C}$ or by sonification with preformed liposomes at $60{ }^{\circ} \mathrm{C}$. In parallel, the antibody fragment is coupled to Mal-PEG ${ }_{2000}$-DSPE micelles and inserted into scTNF-LP by incubation for 30 min at $55^{\circ} \mathrm{C}$ resulting in scTNF-TLP. Because currently no data are available as to the exact nature of the lipid coat, we visualized it in a simplified and speculative way as a lipid bilayer. 
The lipid-coating revealed an effective shielding of the particle surface and a protection from the TNF action which resulted in a more than 20 -fold reduced cytotoxicity on Kym-1 as well as on HT1080 cells. The additional reduction in bioactivity, as seen for anti-FAP targeted lipid-coated scTNF-P (scTNF-TLP) on FAP-negative cells, led to the suggestion of a further reduction in nonspecific binding to cells due to the additional amount of inserted PEG chains into the lipid-coat, further contributing to the protective properties. Importantly, after introduction of micellar anti-FAP scFv-molecules into the lipid coat a reconstitution of selective cytotoxicity towards FAP-expressing cells could be observed. This is in accordance with FACS analyses where only for anti-FAP scTNF-TLP a specific binding to target cells could be shown, whereas scTNF-LP exhibited only marginal binding. Moreover, the flow cytometry analyses revealed a stronger unspecific binding of scTNF-functionalized particles than amino-functionalized particles to these cell lines, which is likely due to TNF receptormediated binding.

Currently the process of uncoating the particles, allowing presentation of TNF to its receptors still remains unknown. A plasma membrane uncoating process after recognition by FAPexpressing cells independent from or possibly even preceding internalization can be discussed. However, this mechanism appears very unlikely inasmuch as the lipid coat shows a good stability under these conditions. An internalization-independent process is also unlikely since first results showed an efficient internalization of ScTNF-TLP upon binding to FAP-positive HT1080 cells. Internalization is likely antigen-dependent as previously shown for amino-functionalized TLP. As discussed before, uptake of anti-FAP immunoliposomes was shown to occur by endocytosis into the endosomal compartment (Baum et al., 2007), which is likely also for anti-FAP scTNF-TLP. Evidence suggests that internalization is a part of the normal signal transduction events for several members of the TNF-receptor superfamily, e.g.TNFR1, after stimulation with their respective ligands (Schneider-Brachert et al., 2004; Micheau \& Tschopp, 2003). As mentioned in the introduction, the distinct signaling pathways of TNFR1 correlate with the formation of two types of complexes. The first complex is formed at the plasma membrane and induces mostly anti-apoptotic signals, whereas the second complex is distinguished by the formation of the TNFR1-associated death inducing signaling complex (DISC) composed of TRADD, FADD (Fas-associated death domain), cFLIP (cellular FLICE-inhibitory protein) and procaspase-8/10 in an early state of receptor endocytosis. This complex is considered to be a prerequisite for the induction of apoptosis (Schneider-Brachert et al., 2004). Therefore, it can be suggested that the membraneexpressed TNFR1 is co-internalized with anti-FAP scTNF-TLP, thus they encounter TNF receptors within the endosome. However, this is only speculative and has to be proven by experimental data, for instance by colocalization studies of targeted nanoparticles and TNF- 
receptors and components of the DISC, respectively.

Although anti-FAP scTNF-TLP showed a significant bioactivity towards FAP-positive cells, they exhibited only approximately $50 \%$ of the cytotoxicity as compared to non-coated scTNFfunctionalized particles, indicating that the uncoating process is only partial. Furthermore, a rather slow uncoating could be conceivable compared to the removal into the lysosomal compartment, thus by TNF degradation no signaling could be proceeded anymore. However, these aspects might be improved by several chemical and genetic engineering approaches. The shielding properties of the lipid coat might be improved by altering the lipid composition, for instance by varying the ratio of phospholipids and cholesterol. Furthermore, phospholipids with a higher transition temperature are also conceivable (Drummond et al., 1999). In this case the conditions of the post-insertion step have to be implemented and potentially the thermal stability of the used target moiety should be proven. By the implementation of active uncoating strategies a local tumor-selective destabilization of the lipid coat from anti-FAP scTNF-TLP could be achieved (Romberg et al., 2008). For instance, a linker with a predetermined cleavage site between the PEG chains and the target moiety can be introduced. Chemical stimuli such as the presence of a low $\mathrm{pH}$ value, reducing agents or enzymatic stimuli can induce cleavage of the linker, proposed to result in a shedding or destabilization of the lipid coat (Levitsky et al., 1998; Zalipsky et al., 1999). For example Hatakeyama et al. designed a protease-sensitive PEG-peptide-DOPE conjugate in which the peptide linker sequence could be cleaved by matrix metallo-proteinases (MMP) which are secreted by tumor cells into extracellular space (Hatakeyama et al., 2007). By means of the incorporation of such PEG chains a shedding of the lipid coat would be achieved after cleavage by tumor-associated proteases. Consequently, coupled TNF is displayed immediately after the binding to target cells and thus TNF action should be restricted to the tumor area. A positive side-effect of the extracellular presentation of TNF could be the recruitment of immune cells like macrophages.

In summary, a multifunctional TNF-functionalized lipid-coated nanoparticle carrier system (scTNF-TLP) was developed that fulfills the expected functional activities. The lipid coat provided an effective shielding of the TNF action, the insertion of a targeting moiety resulted in a targeted delivery of the carrier system which is distinguished by a target restrictedrelease of the TNF action. Thus, a systemic application of TNF combined with a tumor selective activity is conceivable since the body may be protected from the systemic action of TNF, which can lead to severe side effects and even death. Beyond their model character, further in vivo studies have to show whether scTNF-TLP can be developed for therapeutic applications. 
The established carrier system of a targeted lipid-coated polystyrene particle can definitely serve as a versatile platform technology for the development of a variety of multifunctional composite nanoparticles. The miniemulsion technique that is used for the preparation of the embedded polystyrene particle provides the possibility to introduce different therapeutic or diagnostic compounds, e.g. fluorescent dyes (Mysyanovych et al., 2007). Additional, by adding different comonomers during the production process distinct functionalized particle surfaces, e.g. amino- or carboxy-functionalized, can be created. By the combination with the lipid coat endowed with a targeting moiety a manifold application as imaging system or as drug carrier system is thinkable. Moreover, a combination of both approaches is possible and therefore a usage in the diagnostic as well as in the therapeutic field.

Furthermore, the bioactive compound can be exchanged by other members of the TNF superfamily, e.g. FasL or TRAIL. Both pleiotropic proteins are involved in antitumoral responses in the body (Wajant, 2006). TRAIL leads to an induction of apoptosis in various tumor cells, but it is apparently nontoxic to most normal cells and tissues (Kelley \& Ashkenazi, 2004; Ashkenazi, 2002), representing a major advantage as compared to TNF and FasL. Recently some studies reported from enhanced tumoricidal activity of TRAIL in combination with drugs like doxorubicin as compared to the monotherapy (Daniel et al., 2007; Hylander et al., 2005). The here established carrier system is ideally suited for such an approach. A further optimization of the effect could be achieved by an additionally implementation of a targeting effect. The targeting moiety of the carrier system can be variable adapted to the appropriate requirement, e.g. targeting of tumor cells, tumor vasculature or tumor stroma. For instance, the members of the epidermal growth factor receptor (EGFR) tyrosine kinases are a hallmark of numerous human cancers cells and have become a major target for drug delivery and therapy (Anhorn et al., 2008; Song et al., 2008; Tseng et al., 2007; Harries \& Smith, 2002). For a scFv directed against EGFR a further interference with the EGFR signaling was observed, resulting in a proapoptotic effect (Gerspach et al., 2009; Bremer et al., 2005). The carcinoembryonic antigen (CEA) represents a tumor cell target. It is one of the first tumor antigens that was found mostly on tumor cells and not on normal cells (Gold \& Freedman, 1965a; 1965b) and is also one of the most widely used tumor markers for diagnostics (Gold, 1997). Endoglin (CD105) represents another target molecule that has become an attractive tumor vasculature target (Fonsatti et al., 2003) and a few endoglin-targeted immunoliposomes have been described in the literature (Müller et al., 2008; Völkel et al., 2004). Elevated levels of endoglin could be detected in the vasculature of e.g. prostate cancer, breast cancer and melanoma (Liu et al., 2002; Bodey et al., 1998; Dawn et al., 2002).

The here presented approach could also implement particle composed of biocompatible 
and/or biodegradable polymers such as polylactide, polylactide-co-glycoide (PLGA), polyanhydride and polycaprolactone (Mysyanovych et al., 2008; Alexis et al., 2008; Gegnar et al., 2005). However, the used polymers should be stable under physiological conditions for a certain time period, e.g. shown for docetaxel-loaded PLGA-particles (Chan et al., 2009). Biodegradable capsules endowed with a multifunctional lipid coat represent a further possible application of the here established composite carrier system. They can find a use in the diagnostic field, by loading with a fluorescent dye, or in the therapeutic filed by drugloading or by functionalization with a bioactive compound, but also a combination of both is conceivable.

Certainly, the lipid composition and the efficient lipid-coating have to be specially adapted to the physiochemical properties of the different core polymers. Nevertheless, the in this study designed multifunctional composite particles may find various applications in diagnostics and therapy. 


\section{Bibliography}

Ackerson CJ, Jadzinsky PD, Jensen GJ and Kornberg RD. (2006) Rigid, specific, and discrete gold nanoparticle/antibody conjugates. J. Am. Chem. Soc. 128: 2635-2640.

Aggarwal BB. (2003) Signalling pathways of the TNF superfamily: a double-edged sword. Nat.Rev.Immunol. 3 (9): 745-756.

Albrecht H, DeNardo GL and DeNardo SJ. (2006) Monospecific bivalent scFv-SH: effects of linker length and location of an engineered cysteine on production, antigen binding activity and free $\mathrm{SH}$ accessibility. J. Immunol. Methods 310: 100-116.

Alexis F, Rhee JW, Richie JP, Radovic-Moreno AF, Langer R, Farokhzad OC. (2008) New frontiers in nanotechnology for cancer treatment. Urol. Oncol. 26: 74-85.

Al-Jamal WT, Kostarelos K. (2007) Liposome-nanoparticle hybrids for multimodal diagnostic and therapeutic applications. Nanomedicine 2: 85-98.

Allen T, Hansen C and Rutledge J. (1989) Liposomes with prolonged circulation times: factors affecting uptake by reticuloendothelial and other tissues. Biochim Biophys Acta 981: 27-35.

Allen TM, Hansen C, Martin F, Redemann C and Yau-Young A. (1991) Liposomes containing synthetic lipid derivatives of poly (ethylene glycol) show prolonged circulation half-lives in vivo. Biochim Biophys Acta 1066(1): 29-36.

Allen TM, Sapra P, and Moase E. Use of the post-insertion method fort he formation of ligandcoupled liposomes. Cell Mol Biol 7: 889-894, 2002.

Anhorn MG, Wagner S, Kreuter J, Langer K, von Briesen H. (2008) Specific targeting of HER2 overexpressing breast cancer cells with doxorubicin-loaded trastuzumab-modified human serum albumin nanoparticles. Bioconjug Chem. 19(12): 2321-31.

Ashkenazi A. (2002) Targeting death and decoy receptors for tumor-necrosis factor superfamily. Nat Rev Cancer. 2: 420-430.

Augustin HG. (1998) Antiangiogenic tumour therapy: will it work? Trends Pharmacol Sci. 19(6): 21622. Review.

Backmann N, Zahnd C, Huber F, Bietsch A, Plückthun A, Lang HP, Güntheroldt HJ, Hegner M and Gerber C. (2005) A label-free immunosensor array using single-chain antibody fragments. Proc. Natl. Acad. Sci. U.S.A. 102: 14587-14592.

Bangham A, Standish M and Watkins J. (1965). Diffusion of univalent ions across the lamellae of swollen phospholipids. J Mol Biol 13: 238-252.

Banner DW, D'Arcy A, Janes W, Gentz R, Schoenfeld HJ, Broger C, Loetscher H and Lesslauer H. (1993) Crystal structure of the soluble human $55 \mathrm{kd}$ TNF receptorhuman TNF beta complex: implications for TNF receptor activation. Cell 73 (3): 431-445.

Baum P, Müller D, Rüger R, and Kontermann R E. (2007) Single-chain Fv immunoliposomes for the targeting of fibroblast activation protein-expressing tumor stromal cells. J. Drug Target. 15: 399-406.

Bendas G, Krause A, Bakoski U, Vogel J, and Rothe U. Targetability of novel immunoliposomes preparated by a mew antibody conjugation technique. Int J Pharm 181(1): 79-93, 1999.

Bendas G, Rothe U, Scherphof GL, Kamps JA. The influence of repeated injections on pharmacokinetics and biodistribution of different types of sterically stabilised immunoliposomes. Biochim Biophys Acta 1609(1): 63-70, 2003. 
Black RA, Rauch CT, Kozlosky CJ, Peschon JJ, Slack JL, Wolfson MF, Castner BJ, Stocking KL, Reddy P, Srinivasan S, Nelson N, Boiani N, Schooley KA, Gerhart M, Davis R, Fitzner JN, Johnson RS, Paxton RJ, March CJ and Cerretti DP. (1997) A metalloproteinase disintegrin that releases tumour-necrosis factor-alpha from cells. Nature 385 (6618): 729-733.

Bodey B, Bodey B Jr, Siegel SE, Kaiser HE. (1998) Over-expression of endoglin (CD105): a marker of breast carcinoma-induced neo-vascularization. Anticancer Res. 18(5A): 3621-8.

Branschädel M. (2007) Analysis of molecular components essential for the formation of signalling competent TNF-TNFR complexes. Institute of Cellbiology and Immunology, University Stuttgart. Thesis.

Branschädel M, Boschert V and Krippner-Heidenreich A. (2007) Tumour necrosis factors. In:Encyclopedia of Life Sciences, John Wiley \& Sons, Ltd: Chichester.

Bremer E, Samplonius DF, van Genne L, Dijkstra MH, Kroesen BJ, de Leij LF, Helfrich W. (2005) Simultaneous inhibition of epidermal growth factor receptor (EGFR) signaling and enhanced activation of tumor necrosis factor-related apoptosis-inducing ligand (TRAIL) receptor-mediated apoptosis induction by an scFv:sTRAIL fusion protein with specificity for human EGFR. J Biol Chem 280: 1002510033.

O'Brien MER, Wigler N, Inbar M, Rosso R, Grischke E, Santoro A, Catane R, Kieback DG, Tomczak P, Ackland SP, Orlandi F, Mellars L, Alland L, and Tendler C. (2004) Reduced cardiotoxicity and comparable efficacy in a phase III trial of pegylated liposomal doxorubicin $\mathrm{HCl}$ $\left(\mathrm{CAELYX}{ }^{\mathrm{TM}} / \mathrm{Caelyx}^{\circledR}\right)$ versus conventional doxorubicin for first-line treatment of metastatic breast cancer. Ann Oncol 15: 440-449.

Brocks B, Garin-Chesa P, Behrle E, Park JE, Rettig WJ, Pfizenmaier K, Moosmayer D. (2001) Species-crossreactive ScFv against the tumor stroma marker "fibroblast activation protein" selected by phage display from an immunized FAP-/- knock-out mouse. Mol Med. 7(7):461-9.

Bruns P. (1868) Die Heilwirkung des Erysipels auf Geschwulste. Beitr. Klin. Chir. 3: 443-446

Bryde S, Grunwald I, Hammer A, Krippner-Heidenreich A, Schiestel T, Brunner H, Tovar GE, Pfizenmaier K and Scheurich P. (2005) Tumor necrosis factor (TNF)- functionalized nanostructured particles for the stimulation of membrane TNF-specific cell responses. Bioconjug.Chem. 16 (6): 14591467.

Burkle A. (2001) Poly(APD-ribosyl)lation, a DNA damage-driven protein modification and regulator of genomic instability. Cancer Lett. 163: 1-5

Carmona-Ribeiro AM, Lessa M de Moraes. (1999) Interactions between bilayer membranes and latex, Colloids and Surfaces 153: 355-361.

Carswell EA, Old LJ, Kassel RL, Green S, Fiore N and Williamson B. (1975) An endotoxin induced serum factor that causes necrosis of tumors. Proc.Natl.Acad.Sci.U.S.A 72 (9): 3666-3670.

Cegnar M, Kristl J, Kos J. (2005) Nanoscale polymer carriers to deliver chemotherapeutic agents to tumours. Exp. Opin. Biol. Ther. 5: 1557-1569.

Champion JA, Mitragotri S. (2009) Shape induced inhibition of phagocytosis of polymer particles, Pharm. Res. 26: 244-249.

Champion JA, Mitragotri S. (2006) Role of target geometry in phagocytosis, Proc. Natl. Acad. Sci. USA 103: 34930-4934.

Chan FK, Chun HJ, Zheng L, Siegel RM, Bui KL and Lenardo MJ. (2000) A domain in TNF receptors that mediates ligand-independent receptor assembly and signaling. Science 288 (5475): 2351-2354. 
Chan FK. (2000) The pre-ligand binding assembly domain: a potential target of inhibition of tumour necrosis factor receptor function. Ann.Rheum.Dis. 59 Suppl 1: i50-i53.

Chan JM, Zhang L, Yuet KP, Liao G, Rhee JW, Langer R, Farokhzad OC. (2009) PLGA-lecithinPEG core-shell nanoparticles for controlled drug delivery. Biomaterials 30(8): 1627-34.

Cheng WW, Allen TM. (2008) Targeted delivery of anti-CD19 liposomal doxorubicin in B-cell lymphoma: a comparison of whole monoclonal antibody, Fab' fragments and single chain Fv. J Control Release. 126(1): 50-8.

Cheng WW K, Das D, Suresh M and Allen TM. (2007) Expression and purification of two anti-CD19 single chain Fv fragments for targeting of liposomes to CD19-expressing cells. Biochim. Biophys. Acta 1768: 21-29.

Coley WB. (1891) Contribution to the knowledge of Sarcoma. Ann. Surg. 14: 199-220.

Corti A, Fassina G, Marcucci F, Barbanti E and Cassani G. (1992) Oligomeric tumour necrosis factor alpha slowly converts into inactive forms at bioactive levels. Biochem.J. 284 ( Pt 3): 905-910.

Daniel D , Yang B , Lawrence DA, Totpal K, Balter I, Lee WP , Gogineni A , Cole MJ , Yee SF , Ross S , Ashkenazi A. (2007) Cooperation of the proapoptotic receptor agonist rhApo2L/TRAlL with the CD20 antibody rituximab against non-Hodgkin lymphoma xenografts. Blood. $110: 4037-4046$.

Das D, Allen TM, Suresh MR. (2005) Comparative evalutation of two purification methods of antiCD19-c-myc-His ${ }_{6}-$ Cys scFv. Protein Exp Purif 39(2): 1199-1208.

Davis, J. N. Fitzner, R. S. Johnson, R. J. Paxton, C. J. March, and D. P. Cerretti. A metalloproteinase disintegrin that releases tumour-necrosis factor-alpha from cells. Nature 385 (6618):729-733, 1997.

Dawn G, MacKie RM. (2002) Expression of endoglin in human melanocytic lesions. Clin Exp Dermatol. 27(2): 153-6.

Drummond DC, Meyer O, Hong K, Kirpotin DB, Papahadjopoulos D. (1999) Optimizing liposomes for delivery of chemotherapeutic agents to solid tumors. Pharmacol. Rev. 51: 691-743.

Dübel S and Kontermann RE. (2001) Recombinant antibodies. In: Antibody engineering. SpringerVerlag, Heidelberg, pp.3-18.

Duve C, Barsy T, Poole B, Trouet A, Tulkens P and Hoof F. (1975). Lysosomotoric agents. Biochem Pharmacol 23: 2495-2531.

Eck MJ, Beutler B, Kuo G, Merryweather JP and Sprang SR. (1988)Crystallization of trimeric recombinant human tumor necrosis factor (cachectin). J.Biol.Chem. 263 (26): 12816-12819.

Farokhzad OC, Cheng J, Teply BA, Sherifi I, Jon S, Kantoff PW, Richie JP, Langer R. (2006) Targeted nanoparticle-aptamer bioconjugates for cancer chemotherapy in vivo. Proc Natl Acad Sci USA 103(16): 6315-20.

Feinberg B, Kurzrock R, Talpaz M, Blick M, Saks S, Gutterman JU. (1988) A phase I trial of intravenously-administered recombinant tumor necrosis factor-alpha in cancer patients. J Clin Oncol. 6(8): 1328-34.

Filchtinski D, Bee C, Savopol T, Engelhard M, Becker CFW and Herrmann C. (2008) Probing Ras Effector Interactions on Nanoparticle Supported Lipid Bilayers. Bioconjugate Chem. 19(9): 1938-1944.

Fonsatti E, Altomonte M, Arslan P, Maio M. (2003) Endoglin (CD105): a target for anti-angiogenetic cancer therapy. Curr Drug Targets. 4(4): 291-6. Review.

Fotin-Mleczek M, Henkler F, Samel D, Reichwein M, Hausser A, Parmryd L, Scheurich P, 
Schmidt JA and Wajant H. (2002) Apoptotic crosstalk of TNF receptors: TNF-R2-induces depletion of TRAF2 and IAP proteins and accelerates TNF-R1- dependent activation of caspase- 8. J Cell Sci 115: 2757-2770

Fu ZQ, Harrison RW, Reed C, Wu J, Xue YN, Chen MJ and Weber IT. (1995) Model complexes of tumor necrosis factor-alpha with receptors R1 and R2. Protein Eng 8 (12): 1233-1241.

Gabizon A and Papahadjopoulos D. (1992) The role of surface charge and hydrophilic groups on liposome clearance in vivo. Biochim Biophys Acta 1103(1): 94-100.

Gagné J, Desormeaux A, Person S, Tremblay M and Bergeron M. (2002) Targeted delivery of indinavir to HIV-1 primary reservoirs with immunoliposomes. Biochim Biophys Acta, 1558: 198-210.

Garin-Chesa P, Old LJ, and Rettig WJ. (1990) Cell surface glycoprotein of reactive stromal fibroblasts as a potential antibody target in human epithelial cancers. Proc Natl Acad Sci USA 87: 7235-7239.

Gerspach J, Muller D, Munkel S, Selchow O, Nemeth J, Noack M, Petrul H, Menrad A, Wajant H, Pfizenmaier K (2006) Restoration of membrane TNF-like activity by cell surface targeting and matrix metalloproteinase-mediated processing of a TNF prodrug . Cell Death Differ 13: 273-28.

Gerspach J, Wajant H, Pfizenmaier K. (2009) Death ligands desgined to kill: development and application of targeted cancer therapeutics based on proapoptotic TNF family ligands, Results Probl. Cell Differ, Epub ahead of print.

Gold P and Freedman SO.(1965a) Demonstration of Tumor-Specific Antigens in Human Colonic Carcinomata by Immunological Tolerance and Absorption Techniques. J Exp Med 121: 439-462.

Gold P and Freedman So. (1965b) Specific carcinoembryonic antigens of the human digestive system. J Exp Med 122(3): 467-481.

Gold P, Goldenberg NA. (1997) The Carcinoembryonic Antigen (CEA): Past, Present, and Future. McGill Journal of Medicine 3(1).

Gosk S, Gottstein C and Bendas G. (2005) Targeting of immunoliposomes to endothelial cells expressing VCAM: a future strategy in cancer therapy. Int. J. Clin. Pharmacol. Ther. 43: 581-582.

Gottlieb JJ, Washenik K, Chachoua A, Friedman-Kien A. (1997) Treatment of classic Kaposi's sarcoma with liposomal encapsulated doxorubicin. Lancet. 350(9088): 1363-4.

Gregoriadis G, Leathwood PD, Ryman BE. (1971) Enzyme entrapment in liposomes. FEBS Lett 14: 95.

Grell M, Scheurich P, Meager A and Pizenmier K. (1993) TR60 and TR80 tumor necrosis factor (TNF)-receptors can independently mediate cytolysis. Lymphokine Cytokine Res. 12: 143-148

Grell M, Douni E, Wajant H, Lohden M, Clauss M, Maxeiner B, Georgopoulos S, Lesslauer W, Kollias G, Pfizenmaier K and Scheurich P. (1995) The transmembrane form of tumor necrosis factor is the prime activating ligand of the $80 \mathrm{kDa}$ tumor necrosis factor receptor. Cell 83 (5): 793-802.

Grell M, Wajant H, Zimmermann G and Scheurich P. (1998) The type 1 receptor (CD120a) is the high-affinity receptor for soluble tumor necrosis factor. Proc.Natl.Acad.Sci.U.S.A 95 (2): 570-575.

Grell M, Zimmermann G, Gottfried E, Chen CM, Grunwald U, Huang DC, Wu Lee YH, Durkop H, Engelmann H, Scheurich P, Wajant $\mathbf{H}$ and Strasser A. (1999) Induction of cell death by tumour necrosis factor (TNF) receptor 2, CD40 and CD30: a role for TNF-R1 activation by endogenous membrane-anchored TNF. EMBO J. 18 (11): 3034-3043. 
Haginoya K, Kato T, Higuchi M, Shitomi Y, Asakura T, Hayakawa T, Mitsui T and Hori H. (2005) Preparation of stable liposomes using sucrose density centrifugation and their interaction with insecticidal Cry1A toxin of Bacillus thuringiensis. Bull. Facul. Niigata Univ. 57(2): 115-120.

Hansen CB, Kao GY, Moase EH, Zalipsky S, Allen TM. (1995) Attachment of antibodies to sterically stabilized liposomes: Evaluation, comparison and optimization of coupling procedures. Biochim Biophys Acta 1239(2): 133-144.

Harries M, Smith I. (2002) The development and clinical use of trastuzumab (Herceptin). Endocr Relat Cancer. 9(2): 75-85. Review.

Harrington KJ, Mohammadtaghi S, Uster PS, Glass D, Peters AM, Vile RG, and Steward JS. Effective targeting of solid tumors in patients with locally advanced cancers by radiolabeled pegylated liposomes. Clin Canc Res 7: 243-254, 2001.

Hatakeyama H, Akita H, Kogure K, Harashima H. (2007) Development of a novel systemic gene delivery system for cancer therapy with a tumor-specific cleavable PEG-lipid. Yakugaku Zasshi. 127(10): 1549-56.

Hoogenboom HR. (2005) Selecting and screening recombinant antibody libraries. Nat Biotechnol 23(9): 1105-1116.

Horssen van R, Hagen ten TL and Eggermont AM. (2006) TNF-alpha in cancer treatment: molecular insights, antitumor effects, and clinical utility. Oncologist. 11 (4): 397-408.

Hosokawa S, Tagawa T, Niki H, Hirakawa Y, Nohga K, and Nagaike K. Efficacy of immunoliposomes on cancer models in a cell-surface-antigen-density-dependent manner. $\mathrm{Br} J$ Cancer 89(8):1545-1551, 2003.

Hsu H, Huang J, Shu HB, Baichwal V, Goeddel DV. (1996). TNF-dependent recruitment of the protein kinase RIP to the TNF receptor- 1 signaling complex. Immunity. 4: 387-396.

Hu H, Chen D, Liu Y, Deng Y, Yang S, Qiao M, Zhao J and Zhao X. (2006) Target ability and therapy efficacy of immunoliposomes using a humanized antihepatoma disulfidestabilized Fv fragment on tumor cells. J. Pharm. Sci. 95: 192-199.

Hylander BL , Pitoniak R , Penetrante RB , Gibbs JF , Oktay D , Cheng J , Repasky EA. (2005) The anti-tumor effect of Apo2L/TRAIL on patient pancreatic adenocarcinomas grown as xenografts in SCID mice. J Transl Med 3: 22.

Ishida T, Iden DL and Allen TM. (1999) A combinatorial approach to producing sterically stabilized (Stealth) immunoliposomal drugs. FEBS Lett. 460: 129-133.

Jain RK. (1987) Transport of Molecules in the Tumor Interstitium: A Review. Cancer Research 47: 3039-3051.

Jiang Y, Woronicz JD, Liu W, Goeddel DV. (1999). Prevention of constitutive TNF receptor 1 signaling by silencer of death domains. Science 283: 543-546.

Jones EY, Stuart DI and Walker NP. (1990) The structure of tumor necrosis factor - implications for biological functions. J Cell Sci Supp/ 13: 11-18.

Kalluri R and Zeisberg M. Fibroblasts in cancer. Nature 6:392-401, 2006, Review.

Kavalenko A and Wallach D. (2006) If the prophet does not come to the mountain: dynamics of signaling complexes in NF-kappaB activation. Mol Cell 22: 433-436.

Kelley SK and Ashkenazi A. (2004) Targeting death receptors in cancer with Apo2L/TRAlL. Curr Opin Pharmacol. 4: 333-339. 
Kim JH, Kim YS, Park K, Lee S, Nam HY, Min KH, Jo HG, Park JH, Choi K, Jeong SY, Park RW, Kim IS, Kim K, Kwon IC. (2008) Antitumor efficacy of cisplatin-loaded glycol chitosan nanoparticles in tumor-bearing mice. J Control Release. 127(1): 41-9.

Kirpotin DB, Drummond DC, Shao Y, Shalaby MR, Hong K, Nielsen UB, Marks JD, Benz CC, and Park JW. Antibody targeting of long-circuating lipidic nanoparticles does not increase tumour localisation but does increase internalisation in animal models. Cancer Res 66(13): 6732-6740, 2006.

Kolbe A. (2007) Establishing the post-insertion method for the generation of immunoliposomes directed against FAP-expressing tumour stroma cells. diploma thesis.

Koning GA, Morselt HW, Gorter A, Allen TM, Zalipsky S, Scherphof GL, and Kamps JA. Interaction of differently designed immunoliposomes with colon cancer cells and Kupffer cells. An in vitro comparison. Pharm Res 20(8): 1249-1257, 2003.

Kontermann RE, Martineau P, Cummings CE, Karpas A, Allen D, Derbyshire E and Winter G. (1997) Enzyme immunoassays using bispecific diabodies. Immunotechnology 3(2): 137-144.

Kontermann RE, Münkel S, Neumeyer J, Müller D, Branschädel M, Scheurich P and Pfizenmaier K. (2008) A humanized tumor necrosis factor receptor 1 (TNFR1) specific antagonistic antibody for selective inhibition of tumor necrosis factor (TNF) action. J Immunother 31(3): 225-34.

Krippner-Heidenreich A, Tubing F, Bryde S, Willi S, Zimmermann G and Scheurich P. (2002) Control of receptor-induced signaling complex formation by the kinetics of ligand/receptor interaction. J.Biol.Chem. 277 (46): 44155-44163.

Krippner-Heidenreich A, Grunwald I, Zimmermann G, Kuhnle M, Gerspach J, Sterns T, Shnyder SD, Gill JH, Mannel DN, Pfizenmaier K and Scheurich P. (2008) Single-chain TNF, a TNF derivative with enhanced stability and antitumoral activity. J.Immunol. 180 (12): 8176-8183.

Landfester K. Synthesis of colloidal particles in miniemulsions, Ann. Rev. Mater. Res. 36: 231-279, 2006.

Lee AL, Wang Y, Ye WH, Yoon HS, Chan SY, Yang YY. (2008) Efficient intracellular delivery of functional proteins using cationic polymer core/shell nanoparticles. Biomaterials. (9): 1224-32.

Levitsky J, Gurell D, Frishman WH. (1998) Sodium ion/hydrogen ion exchange inhibition: a new pharmacologic approach to myocardial ischemia and reperfusion injury. J Clin Pharmacol 38(10): 88797.

Lonberg N. (2005) Human antibodies from transgenic animals. Nat Biotechnol 23(9): 1117-1125.

Looksley RM, Killeen $\mathbf{N}$ and Lenardo MJ. (2001) The TNF and TNF receptor superfamilies: integrating mammalian biology. Cell 104 (4): 487-501.

Li D, Li P, Li G, Wang J, Wang E. (2009) The effect of nocodazole on the transfection efficiency of lipid-bilayer coated gold nanoparticles. Biomaterials 30(7): 1382-8.

Li X, Yang Y, Ashwell JD. (2002). TNF-RII and C-IAP1 mediate ubiquitination and degradation of TRAF2. Nature 416: 345-347.

Liu Y, Jovanovic B, Pins M, Lee C, Bergan RC.(2002) Over expression of endoglin in human prostate cancer suppresses cell detachment, migration and invasion. Oncogene. 21(54): 8272-81.

Maeda H. (2001) The enhanced permeability and retention (EPR) effect in tumor vasculature: the key role of tumor-selective macromolecular drug targeting. Adv. Enzyme Regul. 41: 189-207.

O'Malley WE, Achinstein B and Shear M. (1962) Action of bacterial polysaccharide on tumors. II. Damage of Sarcoma 37 by serum of mice treated with Serratia marcescens polysaccharide, and 
induced tolerance. J. Natl Cancer Inst. 29: 1196-1175.

Mamot C, Drummond DC, Greiser U, Hong K, Kirpotin DB, Marks JD, and Park JW. Epidermal growth factor receptor (EGFR)- targeted immunoliposomes mediate specific and efficient drug delivery to EGFR- and EGFRvlll-overexpressing tumor cells. Cancer Res 63: 3154-3161, 2003.

Marty C, Scheidegger P, Ballmer-Hofer K, Klemenz R and Schwendener RA. (2001) Production of Functionalized Single-Chain Fv Antibody Fragments Binding to the ED-B Domain of the B-isoform of Fibronectin in Pichia pastoris. Protein Expr Purif 21(1): 156-64.

Marty C, Odermatt B, Schott H, Neri D, Ballmer-Hofer K, Klemenz R, Schwendener RA. (2002) Cytotoxic targeting of F9 teratocarcinoma tumours with anti-ED-B fibronectin scFv antibody modified liposomes. Br J Cancer 87(1): 106-112.

Marty C, Langer-Machova Z, Sigrist S, Schott H, Schwendener RA, Ballmer-Hofer K. (2005) Isolation and characterization of a ScFv antibody specific for tumor endothelial marker 1 (TEM1), a new reagent for targeted tumor therapy. Cancer Lett doi:10.1016/j. canlet.2005.04.029.

Medvedev AE, Sundan A, Espevik T. (1994). Involvement of the tumor necrosis factor receptor p75 in mediating cytotoxicity and gene regulating activities. Eur.J.Immunol. 24: 2842-2849.

Mersmann M, Schmidt A, Rippmann JF, Wuest T, Brocks B, Rettig WJ, Garin-Chesa P, Pfizenmaier K and Moosmayer D. (2001) Human antibody derivatives against the fibroblast activation protein for tumor stroma targeting of carcinomas. Int J Cancer 92(2): 240-248.

Micheau $\mathrm{O}$ and Tschopp J. (2003) Induction of TNF receptor I-mediated apoptosis via two sequential signaling complexes. Cell 114 (2): 181-190.

De Miguel, L. Imbertie, V. Rieumajou, M. Major, R. Kravtzoff, D. Betbeder. (2000) Proofs of the structure of lipid coated nanoparticles (SMBV) used as drug carriers. Pharm. Res. 17: 817-824.

Moghimi SM. (2007) Prolonging the circulation time and modifying the body distribution of intravenously injected polystyrene nanospheres by prior intravenous administration of poloxamine908. A 'hepatic-blockade' event or manipulation of nanosphere surface in vivo? Biochim. Biophys. Acta 1336: 1-6.

Mornet S, Lambert O, Duguet E, Brisson A. (2005) The formation of supported lipid bilayers on silica nanoparticles revealed by cryoelectron microscopy. Nano Lett. 5(2): 281-5.

Moura SP, Carmona-Ribeiro AM. (2006) Biomimetic particles for isolation and reconstitution of receptor function. Cell Biochem. Biophys. 44: 446-452.

Müller D, Trunk G, Sichelstiel A, Zettlitz KA, Quintanilla M, Kontermann RE. (2008) Murine endoglin-specific single-chain Fv fragments for the analysis of vascular targeting strategies in mice. $J$ Immunol Methods. 339(1): 90-8.

Musyanovych A, Rossmanith R, Tontsch C, Landfester K. (2007) Effect of hydrophilic comonomer and surfactant type on the colloidal stability and size distribution of carboxyl- and amino-functionalized polystyrene particles prepared by miniemulsion polymerization. Langmuir 23: 5367-5376.

Musyanovych A, Schmitz-Wienke J, Mailänder V, Walther P, Landfester K. (2008) Preparation of biodegradable polymer nanoparticles by miniemulsion technique and their cell interactions. Macromol Biosci. 8(2): 127-39.

Naismith JH and Sprang SR. (1998) Modularity in the TNF-receptor family. Trends Biochem.Sci. 23 (2): 74-79.

Nash PT and Florin TH. (2005) Tumour necrosis factor inhibitors. Med.J.Aust. 183 (4): 205- 208. 
Nataranja A, Xiong C-Y, Albrecht H, DeNardo GL and DeNardo SJ. (2005) Characterization of sitespecific scFvPEGylation for tumor-targeting pharmaceuticals. Bioconjugate Chem. 16: 113-121.

Nellis DF, Ekstrom DL, Kirpotin DB, Zhu J, Andersson R, Broadt TL, Ouellette TF, Perkins SC, Roach JM, Drummond DC, Hong K, Marks JD, Park JW, and Giardina SL. (2005) Preclinical manufacture of an anti-HER2 scFv-PEG-DSPE, liposome-inserting conjugate. 1. Gram-scale production and purification. BiotechnolProg 21: 205-220.

Niedermeyer J, Scanlan MJ, Garin-Chesa P, Daiber C, Fiebig HH, Old LJ, Rettig WJ, and Schnapp A. (1997) Mouse fibroblast activation protein: molecular cloning, alternative splicing and expression in the reactive stroma of epithelial cancers. Int J Cancer 71: 383-389.

Nielsen UB, Kirpotin DB, Pickering EM, Hong K, Park JW, Shalaby MR, Shao Y, Benz CC and Marks JD. (2002) Therapeutic efficacy of anti-ErbB2 immunoliposomes targeted by a phage antibody selected for cellular endocytosis. Biochim. Biophys. Acta 1591: 109-108.

Northfelt DW, Martin FJ, Working P, Volberding PA, Russell J, Newman M, Amantea MA and Kaplan LD. (1996) Doxorubicin encapsulated in liposomes containing surface-bound polyethylene glycol: pharmacokinetics, tumor localization, and safety in patients with AIDS-related Kaposi's sarcoma. J Clin Pharmacol 36(1): 55-63.

Paciotti GF, Myer L, Weinreich D, Goia D, Pavel N, McLaughlin RE, Tamarkin L. (2004) Colloidal Gold: A Novel Nanoparticle Vector for Tumor Directed Drug Delivery. Drug Delivery 11(3): 169-83.

Papahadjopoulos D, Allen TM, Gabizon A, Mayhew E, Matthay K, Huang SK, Lee KD, Woodle MC, Lasic DD, Redemann C, et al. (1991) Sterically stabilized liposomes: improvements in pharmacokinetics and antitumor therapeutic efficacy. Proc Natl Acad Sci USA 88(24):11460-4.

Park, JE, Lenter, MC, Zimmermann, RN, Garin-Chesa, P, Old, LJ and Rettig, WJ. (1999) Fibroblast activation protein, a dual specificity serine protease expressed in reactive human tumor stromal fibroblasts. J Biol Chem 274(51): 36505-36512.

Park JW, Hong K, Carter P, Asgari H, Guo LY, Keller GA, Wirth C, Shalaby R, Kotts C, Wood WI, Papahadjopoulos D, Benz CC. (1995) Development of anti-p185HER2 immunoliposomes for cancer therapy. Proc Natl Acad Sci USA 92(5): 1327-1331.

Park JW, Hong K, Kirpotin DB, Colbern G, Shalaby R, Baselga J, Shao Y, Nielsen UB, Marks JD, Moore D, Papahadjopoulos D, Benz CC. (2002) Anti-HER2 immunoliposomes: enhanced efficacy attributable to targeted delivery. Clin. Cancer Res. 8: 1172-1181.

Park JW, Kirpotin DB, Hong K, Shalaby R, Shao Y, Nielsen UB, Marks JD, Papahadjopoulos D, Benz CC. (2001) Tumor targeting using anti-HER2 immunoliposomes. J Control Release 74(1-3): 95113.

Pastorino F, Brignole C, Marimpietri D, Sapra P, Moase EH, Allen TM, and Ponzoni M. (2003) Doxorubicin-loaded Fab' fragments of anti-disialoganglioside immunoliposomes selectively inhibit the growth and dissemination of human neuroblastoma in nude mice. Cancer Res 63(1): 86-92.

Pennica D, Hayflick JS, Bringman TS, Palladino MA and Goeddel DV. (1985) Cloning and expression in Escherichia coli of the cDNA for murine tumor necrosis factor. Proc.Natl.Acad.Sci.U.S.A 82 (18): 6060-6064.

Phillips NC, Dahman J. (1995) Immunogenicity of immunoliposomes: reactivity against speciesspecific IgG and liposomal phospholipids. Immunol Lett. 45(3): 149-52.

Pulkkinen M, Pikkarainen J, Wirth T, Tarvainen T, Haapa-aho V, Korhonen H, Seppälä J, Jiirvinen K. (2008) Three-step tumor targeting of paclitaxel using biotinylated PLA-PEG nanoparticles and avidin-biotin technology: Formulation development and in vitro anticancer activity. European Journal of Pharmaceutics and Biopharmaceutics 70: 66-74. 
Puu G, Artursson E, Gustafson I, Lundstrom M, Jass J. (2000) Distribution and stability of membrane proteins in lipid membranes on solid supports. Biosens. Bioelectron. 15: 31-41.

Ramirez-Montagut T, Blachere NE, Sviderskaya EV, Bennett DC, Rettig WJ, Garin-Chesa P and Houghton AN. (2004) FAPalpha, a surface peptidase expressed during wound healing, is a tumor suppressor. Oncogene 23(32): 5435-5446.

Reed C, Fu ZQ, Wu J, Xue YN, Harrison RW, Chen MJ and Weber IT. (1997) Crystal structure of TNF-alpha mutant R31D with greater affinity for receptor R1 compared with R2. Protein Eng 10 (10): 1101-1107.

Rettig WJ, Garin-Chesa P, Healey JH, Su SL, Ozer HL, Schwab M, Albino AP, and Old LJ. (1993) Regulation and heteromeric structure of the fibroblast activation protein in normal and transformed cells of mesenchymal and neuroectodermal origin. Cancer Res 53(14): 3327-3335.

Riedl SJ and Shi Y. (2004) Molecular mechanisms of caspase regulation during apoptosis. Nat.Rev.Mol.Cell Biol. 5 (11): 897-907.

Rijcken CJ, Soga O, Hennink WE, van Nostrum CF. (2007) Triggered destabilisation of polymeric micelles and vesicles by changing polymers polarity: an attractive tool for drug delivery. $J$ Control Release. 120(3): 131-48. Review.

Rodal S, Skretting G, Garred O, Vilhardt F, van Deurs B and Sandvig K. (1999) Extraction of cholesterol with methyl-beta-cyclodextrin perturbs formation of clathrincoated endocytic vesicles. Mol Biol Cell, 10: 961-974.

Romberg B, Hennink WE, Storm G. (2007) Sheddable coatings for long-circulating nanoparticles, Pharm. Res. 25: 55-71.

Rothe M, Wong SC, Henzel WJ and Goeddel DV. (1994) A novel family of putative signal transducers associated with the cytoplasmic domain of the $75 \mathrm{kDa}$ tumor necrosis factor receptor. Cell 78 (4): 681-692.

Rüger R. (2008) Liposomen zur Zielsteuerung von Arzneistoffen - Oberflächenmodifikationen. Thesis.

Rüger R, Müller D, Fahr A, and Kontermann RE. (2006) In vitro characterization of binding and stability of single-chain Fv Ni-NTA-liposomes. J Drug Target 14(8): 576-582.

Sapra P, Allen TM (2002) Internalizing antibodies are necessary for improved therapeutic efficacy of antibody-targeted liposomal drugs. Cancer Res 62(24): 7190-7194.

Sapra P, Moase EH, Ma J, Allen TM. (2004) Improved therapeutic responses in a xenograft model of human B lymphoma (Namalwa) for liposomal vincristine versus liposomal doxorubicin targeted bia anti-CD19 IgG2a or Fab' fragments, Clin. Cancer Res. 10: 100-1111.

Scaffidi C, Fulda S, Srinivasan A, Friesen C, Li F, Tomaselli KJ, Debatin KM, Krammer PH and Peter ME. (1998) Two CD95 (APO-1/Fas) signaling pathways. EMBO J. 17 (6): 1675-1687.

Scanlan MJ, Raj BKM, Calvo B, Garin-Chesa P, Sanz-Moncasi MP, Healey JH, Old LJ, and Rettig WJ. (1994) Molecular cloning of fibroblast activation protein $\alpha$, amember of the serine protease family selectively expressed in stromal fibroblasts of epithelial cancers. Proc Natl Acad Sci USA 91: 56575661.

Schäfer J, Sitterberg J, Ehrhardt C, Ravi Kumar NMV and Bakowsky U. (2008) A new Drug Vehicle - Lipid Coated Biodegradable Nanoparticles. Advances in Science and Technology 57: 148153.

Schmidt A, Muller D, Mersmann M, Wuest T, Gerlach E, Garin-Chesa P, Rettig WJ, Pfizenmaier K and Moosmayer D. (2001) Generation of human high-affinity antibodies specific for the fibroblast 
activation protein by guided selection. Eur J Biochem 268(6): 1730-1738.

SchmiedI A, Breitling F, Winter CH, Queitsch I and Dübel S. (2000) Effects of unpaired cyteines on yield, solubility and activity of different recombinant antibody constructs expressed in E. coli. J. Immunol. Methods 242: 101-114.

Schneider-Brachert W, Tchikov V, Neumeyer J, Jakob M, Winoto-Morbach S, Held-Feindt J, Heinrich M, Merkel O, Ehrenschwender M, Adam D, Mentlein R, Kabelitz D and Schutze S. (2004) Compartmentalization of TNF receptor 1 signaling: internalized TNF receptosomes as death signalling vesicles. Immunity. 21 (3): 415-428.

Schneider P, Holler N, Bodmer JL, Hahne M, Frei K, Fontana A and Tschopp J. (1998) Conversion of membrane-bound Fas(CD95) ligand to its soluble form is associated with downregulation of its proapoptotic activity and loss of liver toxicity. J.Exp.Med. 187 (8): 1205-1213.

Schooneveld van MM, Vucic E, Koole R, Zhou Y, Stocks J, Cormode DP, Tang CY, Gordon RE, Nicolay K, Meijerink A, Fayad ZA, Mulder WJM. (2008) Improved biocompatibility and pharmacokinetics of silica nanoparticles by means of a lipid coating: a multimodality investigation. Nano Lett. 8: 2517-2525.

Sebban H, Yamaoka S and Courtois G. (2006) Postranslational modifications of NEMO and its partners in NF-kappaB signaling. Trends Cell Biol 16: 569-577.

Senarath-Yapa MD, Phimphivong S, Coym JW, Wirth MJ, Aspinwall CA, Saavedra SS. (2007) Preparation and characterization of poly(lipid)-coated, fluorophore-doped silica nanoparticles for biolabeling and cellular imaging. Langmuir 23: 12624-12633.

Shanbhag AS, Jacobs JJ, Black J, Galante JO, Glant TT. (1994) Macrophage/particle interactions: Effect of size, composition and surface area. J. Biomed. Mater. Res. 28: 81-90.

Shen Z, Stryker GA, Mernaugh RL, Yu L, Yan H and Zeng X. (2005) Single-chain fragment variable antibody piezoimmunosensors. Anal. Chem. 77: 797-805.

Shi Y. (2002). Mechanisms of Caspase Activation and Inhibition during Apoptosis. Mol.Cell 9: 459470.

Shinkai M, Le B, Honda H (2001) Targeting hyperthermia for renal cell carcinoma using human MN antigen-specific magnetoliposomes. Jpn. J. Cancer Res. 2: 1138-1145.

Siegel RM, Frederiksen JK, Zacharias DA, Chan FK, Johnson M, Lynch D, Tsien RY and Lenardo MJ. (2000) Fas preassociation required for apoptosis signaling and dominant inhibition by pathogenic mutations. Science 288 (5475): 2354-2357.

Sieczkarski S and Whittaker G. (2002). Dissecting virus entry via endocytosis. J Gen Virol 83: 15351545.

Song S, Liu D, Peng J, Sun Y, Li Z, Gu JR, Xu Y.(2008) Peptide ligand-mediated liposome distribution and targeting to EGFR expressing tumor in vivo. Int J Pharm. 363(1-2): 155-61.

Terpe K. (2003) Overview of tag protein fusions: from molecular and biochemical fundamentals to commercial systems. Appl. Microbiol. Biotechnol. 60: 523-533.

Torchilin VP. (2007) Targeted pharmaceutical nanocarriers for cancer therapy and imaging. AAPS J. 11: E128-147.

Tseng CL, Wang TW, Dong GC, Yueh-Hsiu Wu S, Young TH, Shieh MJ, Lou PJ, Lin FH. (2007) Development of gelatin nanoparticles with biotinylated EGF conjugation for lung cancer targeting. Biomaterials 28(27): 3996-4005. 
Tsuruta LR, Quintilio W, Costa MH, Carmona-Ribeiro AM. (1997) Interactions between cationic liposomes and an antigenic protein: the physical chemistry of the immunoadjuvant action. $J$ Lipid Res 38(10): 2003-11.

Verma IM, Steverson JK, Schwarz EM et al. (1995) Rel/NF-kappa B/l kappa B family: intimate tales of association and dissociation. Genes Dev. 9: 2723-2735.

Völkel T, Hölig P, Merdan T, Müller R, Kontermann RE. (2004) Targeting of immunoliposomes to endothelial cells using a single-chain Fv fragment directed against human endoglin (CD105). Biochim Biophys Acta 1663(1-2): 158-166.

Wang L, Rothberg K and Anderson R. (1993) Mis-assembly of clathrin lattices on endosomes reveals a regulatory switch for coated pit formation. J Cell Biol 123: 1107-1117.

Wang X. (2001) The expanding role of mitochondria in apoptosis. Genes Dev. 15: 2922-2933.

Wajant H, Henkler F and Scheurich P. (2001) The TNF-receptor-associated factor family: scaffold molecules for cytokine receptors, kinases and their regulators. Cell Signal. 13 (6): 389-400.

Wajant H, Moosmayer D, Wuest T, Bartke T, Gerlach E, Schonherr U, Peters N, Scheurich P and Pfizenmaier K. (2001) Differential activation of TRAIL-R1 and -2 by soluble and membrane TRAIL allows selective surface antigen-directed activation of TRAIL-R2 by a soluble TRAIL derivative. Oncogene 20 (30): 4101-4106.

Wajant H, Pfizenmaier K, Scheurich P. (2003). Tumor necrosis factor signaling. Cell Death.Differ. 10: $45-65$.

Wajant H, Gerspach J and Pfizenmaier K. (2005) Tumor therapeutics by design: targeting and activation of death receptors. Cytokine Growth Factor Rev. 16 (1): 55-76.

Wajant H. (2006) CD95L/FasL and TRAIL in tumor surveillance and cancer therapy. Cancer Trat Res. 130: $141-165$.

Weingärtner M, Siegmund D, Schlecht U, Fotin-Mleczek M, Scheurich P, Wajant H. (2002) Endogenous membrane TNF is a potent amplifier of TNF-R1-mediated apoptosis. J Biol Chem. 277: 34853-34859

Yang L, Mao H, Wang YA, Cao Z, Peng X, Wang X, Duan H, Ni C, Yuan Q, Adams G, Smith MQ, Wood WC, Gao X, Nie S. (2009) Single chain epidermal growth factor receptor antibody conjugated nanoparticles for in vivo tumor targeting and imaging. Small 5(2): 235-43.

Zalipsky S, Qazen M, Walker JA 2nd, Mullah N, Quinn YP, Huang SK. (1999) New detachable poly(ethylene glycol) conjugates: cysteine-cleavable lipopolymers regenerating natural phospholipid, diacyl phosphatidylethanolamine. Bioconjug Chem 10(5): 703-7. 


\section{Appendix}

\section{ScFv' $36 \mathrm{HC2}$}

1 atgaatacc tattgcctac ggcagccget ggattgttat tactcgcggc ccagccggcc atggcccagg tgcagctgaa

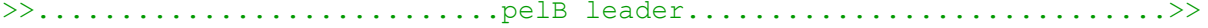

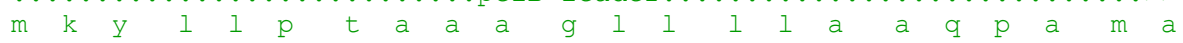
$>>$. scFv36...> q $\quad \mathrm{V} \quad \mathrm{q} \quad \mathrm{l}$

81 gcagtctgga gctgaactgg tgaaacccgg ggcatcagtg aagctgtcct gcaagacttc tggctacacc ttcactgaaa

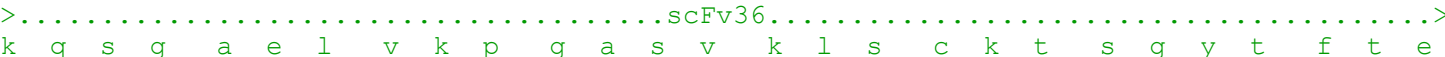

161 atattataca ctgggtaaag cagaggtctg ggcagggtct tgagtggatt gggtggtttc accctggaag tggtagtata

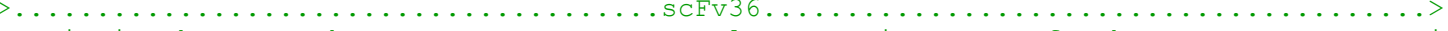

241 aagtacaatg agaaattcaa ggacaaggcc acattgactg cggacaaatc ctccagcaca gtctatatgg agcttagtag

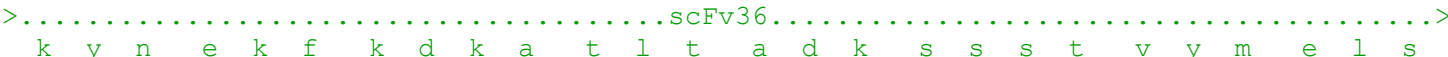

321 attgacatct gaagactctg cggtctattt ctgtgcaaga cacggaggaa ctgggcgagg agctatggac tactggggtc

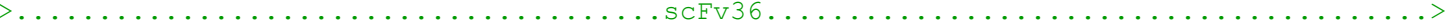

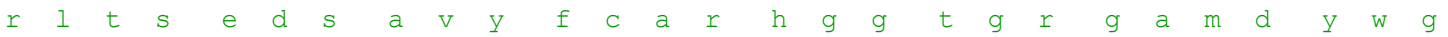

401 aaggaacctc agtcaccgtc tcgagtggtg gaggcggttc aggcggaggt ggctctggcg gtagtgcaca aattctgatg Linker

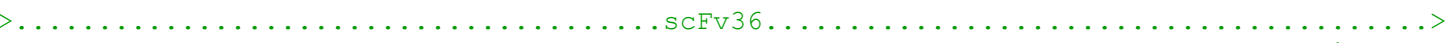

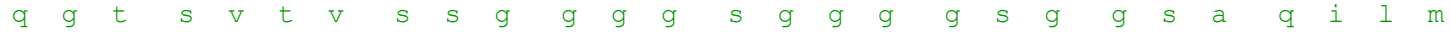

481 acccagtctc ctgcttcctc agttgtatct ctggggcaga gggccaccat ctcatgcagg gccagcaaaa gtgtcagtac

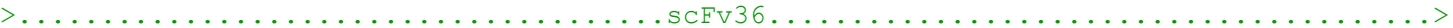

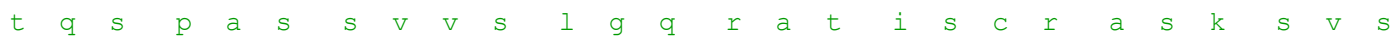

561 atctgcctat agttatatgc actggtacca acagaaacca ggacagccac ccaaactcct catctatctt gcatccaacc

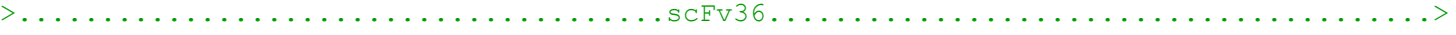

641 tagaatctgg ggtccctccc aggttcagtg gcagtgggtc tgggacagac ttcaccctca acatccaccc tgtggaggag

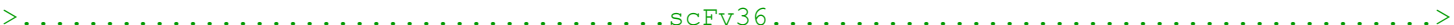

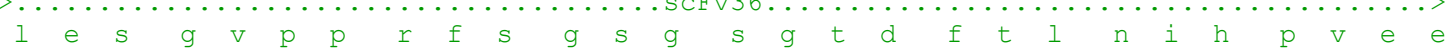

721 gaggatgctg caacctatta ctgtcagcac agtagggagc ttccgtacac gttcggaggg gggaccaaac tggaaatcaa

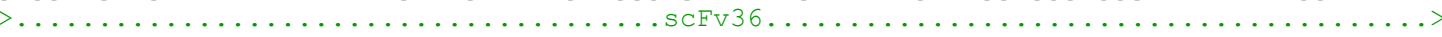

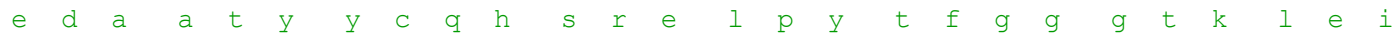

801 acgtgcgcat catcaccatc accatggcgg atcgagtggc tcaggatgct aa HisTag Cys $>\ldots \ldots \ldots \ldots \ldots \ldots \ldots \ldots \ldots \ldots \ldots \ldots \ldots+\ldots \ldots$ k $r$ a $h \begin{array}{lllllllllllllll} & h & h & h & h & h & g & g & s & s & g & s & g & c & -\end{array}$

\section{scFv' $36 \mathrm{HC} 3$}

1 atgaaatacc tattgcctac ggcagccget ggattgttat tactcgcggc ccagccggcc atggcccagg tgcagctgaa

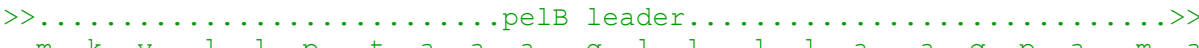

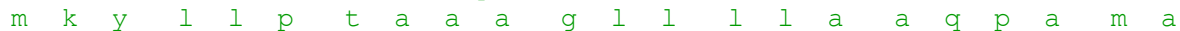

$>>$. ScFv36...>

q $\quad \mathrm{V} \quad \mathrm{q} \quad \mathrm{l}$

81 gcagtctgga gctgaactgg tgaaacccgg ggcatcagtg aagctgtcct gcaagacttc tggctacacc ttcactgaaa

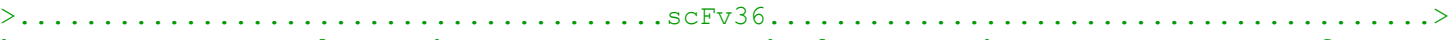

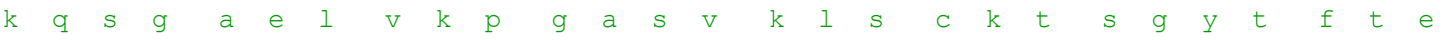

161 atattataca ctgggtaaag cagaggtctg ggcagggtct tgagtggatt gggtggtttc accctggaag tggtagtata

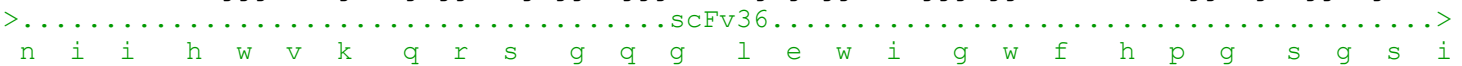

241 aagtacaatg agaaattcaa ggacaaggcc acattgactg cggacaaatc ctccagcaca gtctatatgg agcttagtag

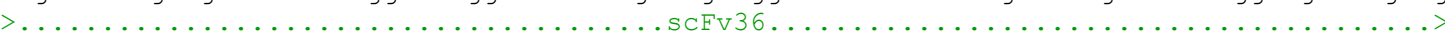

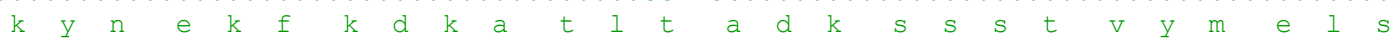


321 attgacatct gaagactctg cggtctattt ctgtgcaaga cacggaggaa ctgggcgagg agctatggac tactggggtc

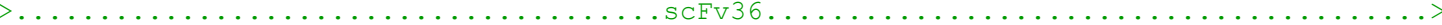

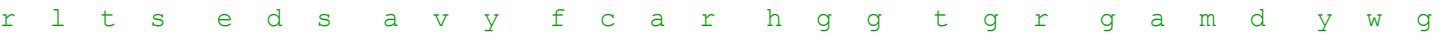

401 aaggaacctc agtcaccgtc tcgagtggtg gaggcggttc aggcggaggt ggctctggcg gtagtgcaca aattctgatg Linker

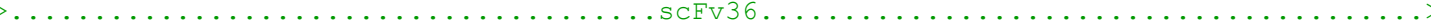

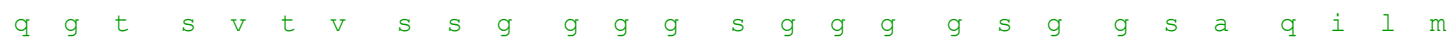

481 acccagtctc ctgcttcctc agttgtatct ctggggcaga gggccaccat ctcatgcagg gccagcaaaa gtgtcagtac

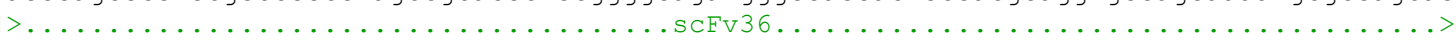

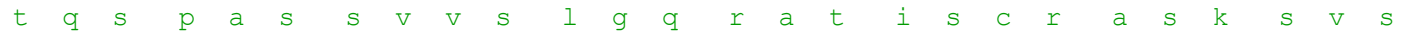

561 atctgcctat agttatatgc actggtacca acagaaacca ggacagccac ccaaactcct catctatctt gcatccaacc

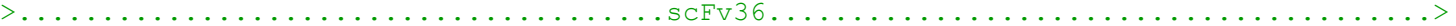

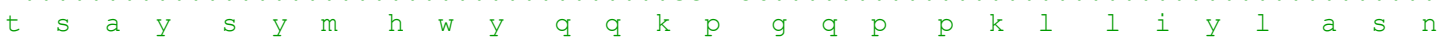

641 tagaatctgg ggtccctccc aggttcagtg gcagtgggtc tgggacagac ttcaccctca acatccaccc tgtggaggag

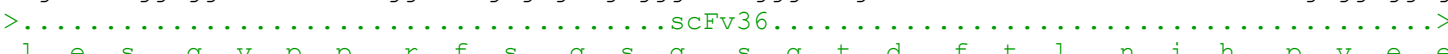

721 gaggatgctg caacctatta ctgtcagcac agtagggagc ttccgtacac gttcggaggg gggaccaaac tggaaatcaa

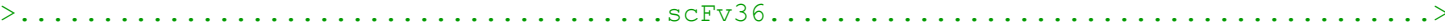

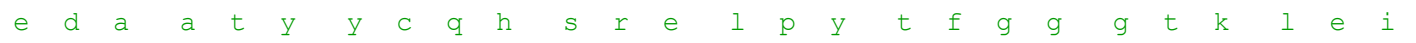

801 acgtgcgcat catcaccatc accatggcgg atcgagtggc tcatgcggat gtagttgcta a HisTag Cys Cys Cys

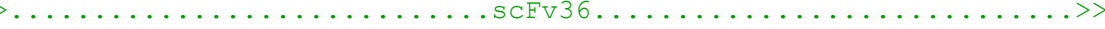

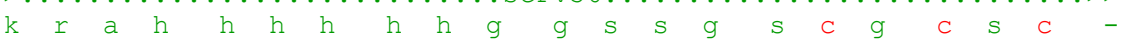

\section{scFv' 36 HC4}

1 atgaaatacc tattgcctac ggcagccget ggattgttat tactcgcgge ccagccggce atggcccagg tgcagctgaa

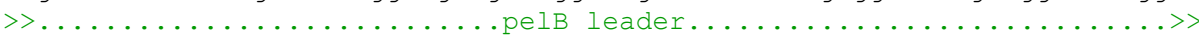

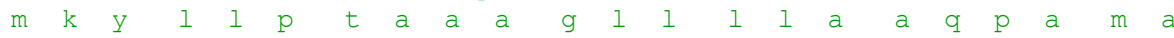
$>>$. ScFv36...> q $\quad \mathrm{V} \quad \mathrm{q} \quad \mathrm{l}$

81 gcagtctgga gctgaactgg tgaaacccgg ggcatcagtg aagctgtcct gcaagacttc tggctacacc ttcactgaaa

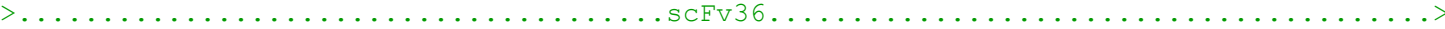

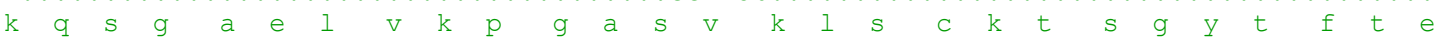

161 atattataca ctgggtaaag cagaggtctg ggcagggtct tgagtggatt gggtggtttc accctggaag tggtagtata

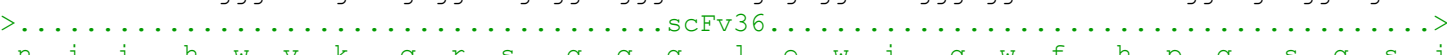

241 aagtacaatg agaattcaa ggacaaggcc acattgactg cggacaatc ctccagcaca gtctatatgg agcttagtag

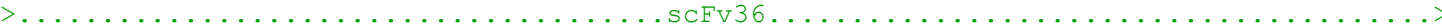

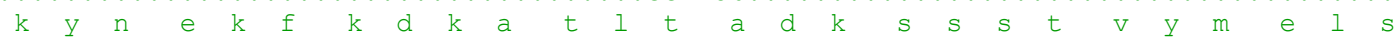

321 attgacatct gaagactctg cggtctattt ctgtgcaaga cacggaggaa ctgggcgagg agctatggac tactggggtc

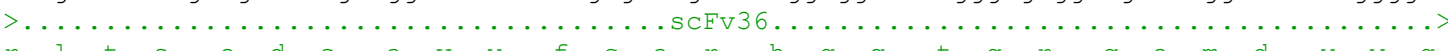

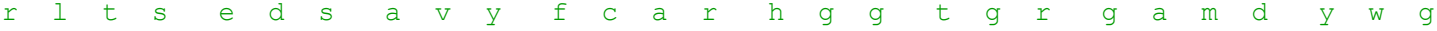

401 aaggaacctc agtcaccgtc tcgagtggtg gaggcggttc aggcggaggt ggctctggcg gtagtgcaca aattctgatg Linker

481 acccagtctc ctgcttcctc agttgtatct ctggggcaga gggccaccat ctcatgcagg gccagcaaaa gtgtcagtac

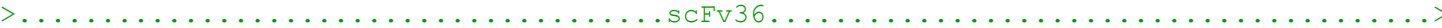

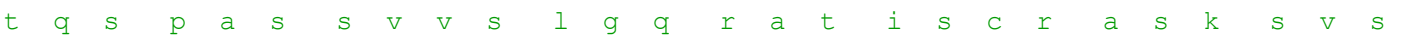

561 atctgcctat agttatatgc actggtacca acagaaacca ggacagccac ccaaactcct catctatctt gcatccaacc

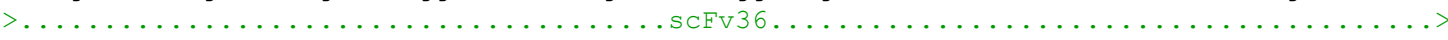

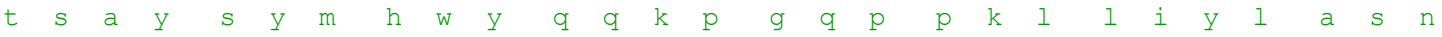

641 tagaatctgg ggtccctccc aggttcagtg gcagtgggtc tgggacagac ttcaccctca acatccaccc tgtggaggag

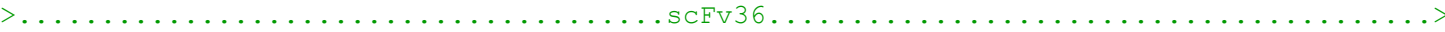

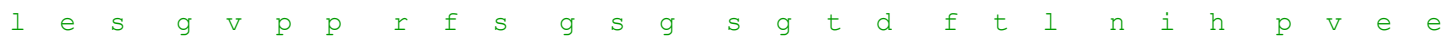

721 gaggatgctg caacctatta ctgtcagcac agtagggagc ttccgtacac gttcggaggg gggaccaaac tggaaatcaa 


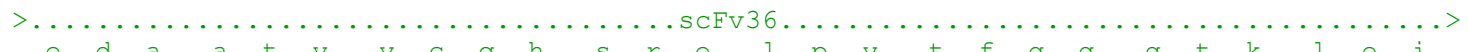

801 acgtgcgcat catcaccatc accacggcgg atccagcggc ggatccagcg gctccggatg ctaa HisTag Cys

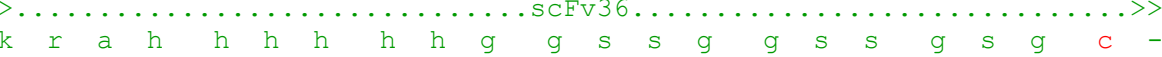

\section{scFv' 36 LC1}

1 atgaaatacc tattgcctac ggcagccget ggattgttat tactcgcgge ccagccggcc atggcccagg tgcagctgaa

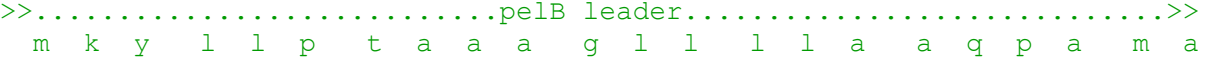
$>>$. ScFv36...>

81 gcagtctgga getgaactgg tgaaacccgg ggcatcagtg aagctgtcct gcaagacttc tggctacacc ttcactgaaa 

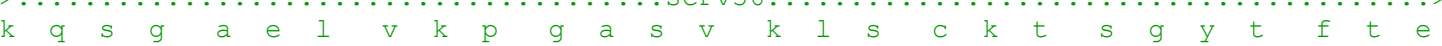

161 atattataca ctgggtaaag cagaggtctg ggcagggtct tgagtggatt gggtggtttc accctggaag tggtagtata

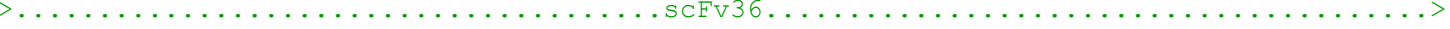

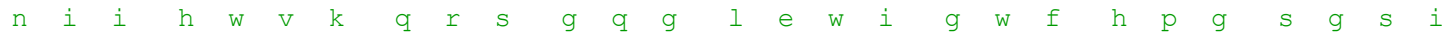

241 aagtacaatg agaaattcaa ggacaaggcc acattgactg cggacaaatc ctccagcaca gtctatatgg agcttagtag

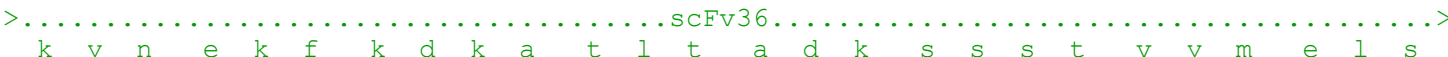

321 attgacatct gaagactctg cggtctattt ctgtgcaaga cacggaggaa ctgggcgagg agctatggac tactggggtc

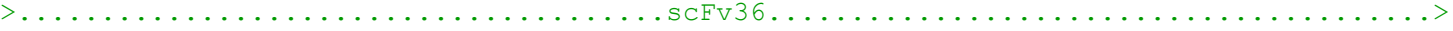

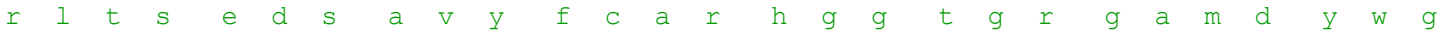

401 aaggaacctc agtcaccgtc tcgagttgcg gaggcggttc aggcggaggt ggctctggcg gtagtgcaca aattctgatg Cys Linker

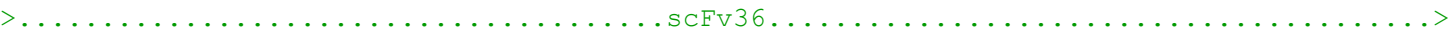

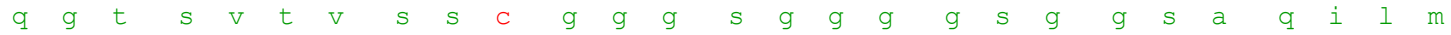

481 acccagtctc ctgcttcctc agttgtatct ctggggcaga gggccaccat ctcatgcagg gccagcaaaa gtgtcagtac

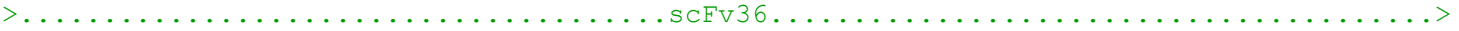

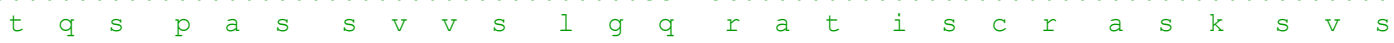

561 atctgcctat agttatatgc actggtacca acagaaacca ggacagccac ccaaactcct catctatctt gcatccaacc

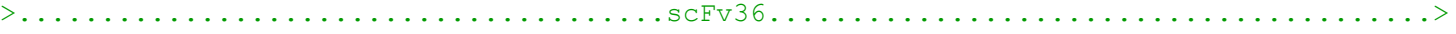

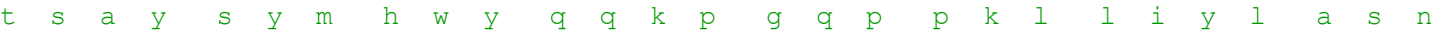

641 tagaatctgg ggtccctccc aggttcagtg gcagtgggtc tgggacagac ttcaccetca acatccaccc tgtggaggag

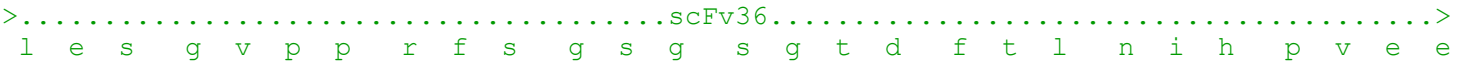

721 gaggatgctg caacctatta ctgtcagcac agtagggagc ttccgtacac gttcggaggg gggaccaagc tggaaataaa

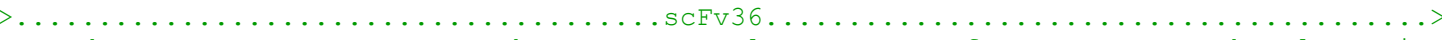

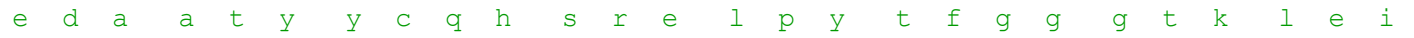

801 acgggcggcc gcagaacaaa aactcatctc agaagaggat ctgaatgggg ccgcacatca ccatcatcac cattaataag His-Taq $>\ldots \ldots \ldots \ldots \ldots \ldots \ldots \ldots \ldots \ldots \ldots \ldots \ldots \ldots \ldots \ldots \ldots \ldots \ldots \ldots$

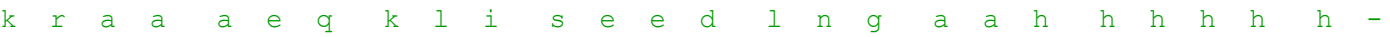

\section{scFv' 36 LC2}

1 atgaaatacc tattgcctac ggcagccgct ggattgttat tactcgcggc ccagccggcc atggcccagg tgcagctgaa

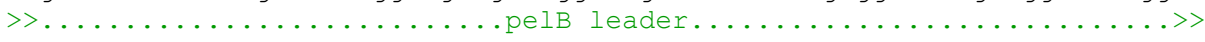

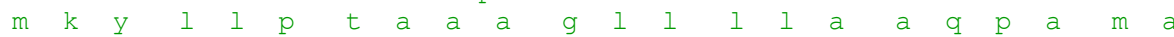
$>$. . scFv36...>

q $\quad \mathrm{V} \quad \mathrm{q} \quad 1$

81 gcagtctgga gctgaactgg tgaaacccgg ggcatcagtg aagctgtcct gcaagacttc tggctacacc ttcactgaaa

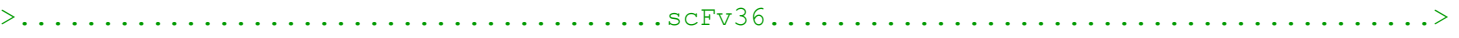

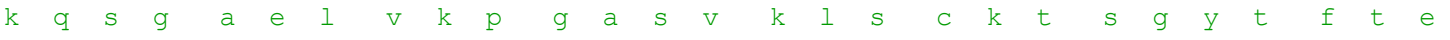

161 atattataca ctgggtaaag cagaggtctg ggcagggtct tgagtggatt gggtggtttc accctggaag tggtagtata 


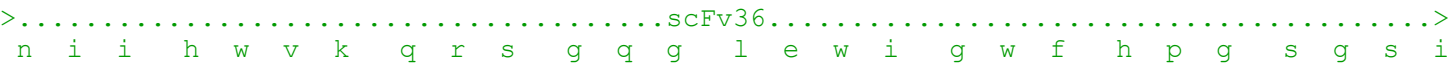

241 aagtacaatg agaaattcaa ggacaaggcc acattgactg cggacaaatc ctccagcaca gtctatatgg agcttagtag

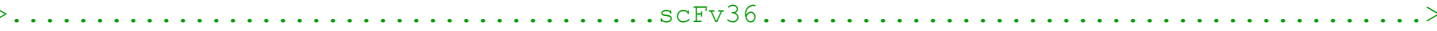

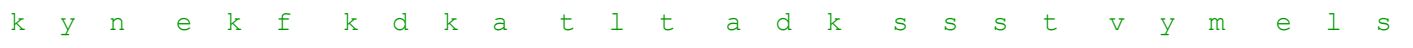

321 attgacatct gaagactctg cggtctattt ctgtgcaaga cacggaggaa ctgggcgagg agctatggac tactggggtc

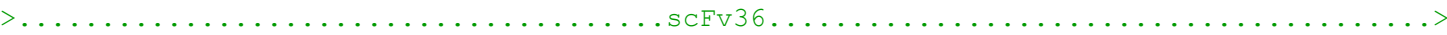

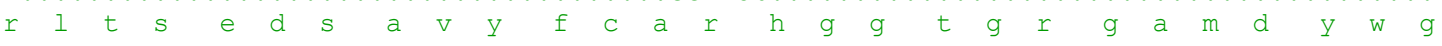

401 aaggaacctc agtcaccgtc tcgagtggtt gcggcggttc aggcggaggt ggctctggcg gtagtgcaca aattctgatg

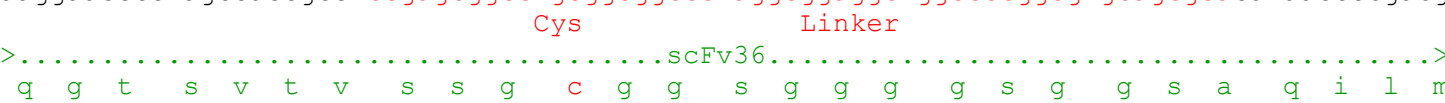

481 acccagtctc ctgcttcctc agttgtatct ctggggcaga gggccaccat ctcatgcagg gccagcaaaa gtgtcagtac

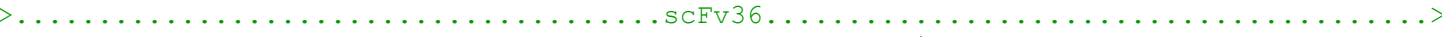

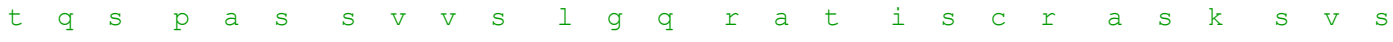

561 atctgcctat agttatatgc actggtacca acagaaacca ggacagccac ccaaactcct catctatctt gcatccaacc

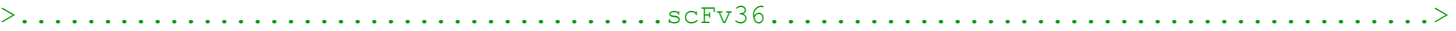

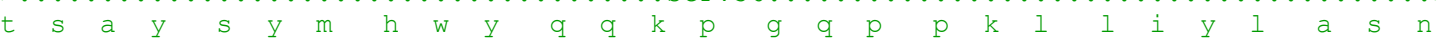

641 tagaatctgg ggtccetccc aggttcagtg gcagtgggtc tgggacagac ttcaccetca acatccaccc tgtggaggag

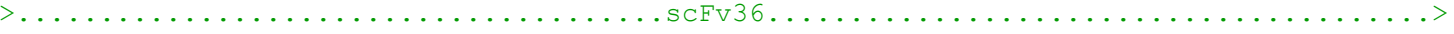

721 gaggatgctg caacctatta ctgtcagcac agtagggagc ttccgtacac gttcggaggg gggaccaagc tggaaataaa

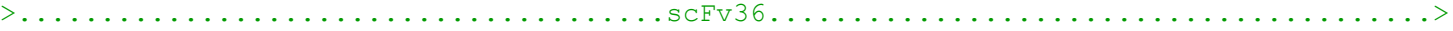

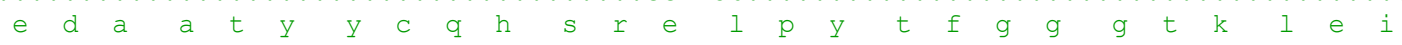

801 acgggcggcc gcagaacaaa aactcatctc agaagaggat ctgaatgggg ccgcacatca ccatcatcac cattaataag His-Taq

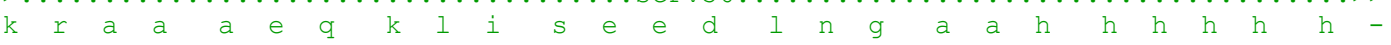

\section{scFv' 36 LC3}

1 atgaaatacc tattgcctac ggcagccgct ggattgttat tactcgcggc ccagccggcc atggcccagg tgcagctgaa $>\ldots \ldots \ldots \ldots \ldots \ldots \ldots \ldots \ldots \ldots \ldots \ldots \ldots \ldots \ldots \ldots$

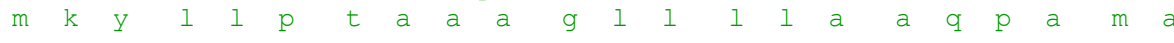
$>$. . scFv36...> q $\quad \mathrm{V} \quad \mathrm{q} \quad 1$

81 gcagtctgga gctgaactgg tgaaacccgg ggcatcagtg aagctgtcct gcaagacttc tggctacacc ttcactgaaa

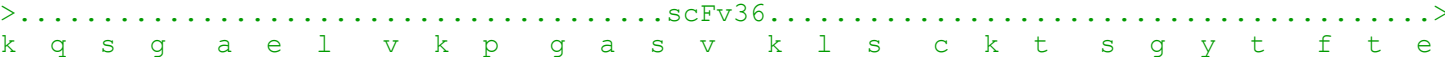

161 atattataca ctgggtaaag cagaggtctg ggcagggtct tgagtggatt gggtggtttc accctggaag tggtagtata

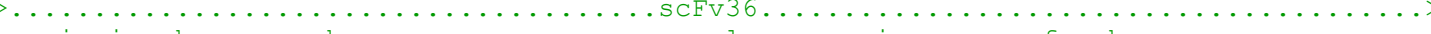

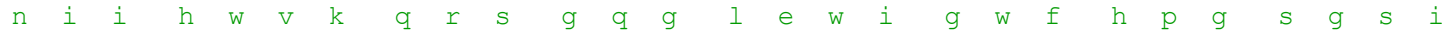

241 aagtacaatg agaaattcaa ggacaaggcc acattgactg cggacaatc ctccagcaca gtctatatgg agcttagtag

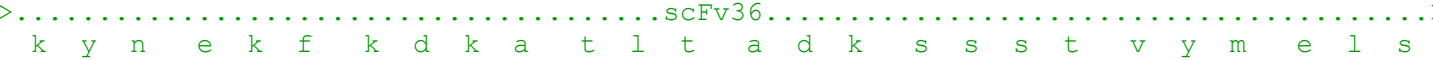

321 attgacatct gaagactctg cggtctatt ctgtgcaaga cacggaggaa ctgggcgagg agctatggac tactggggtc

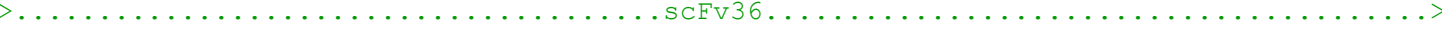

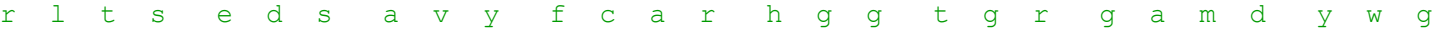

401 aaggaacctc agtcaccgtc tcgagtggtg gatgcggttc aggcggaggt ggctctggcg gtagtgcaca aattctgatg Cys Linker

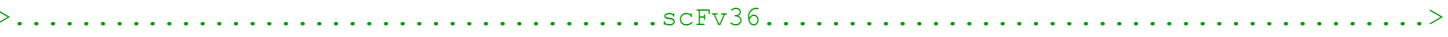

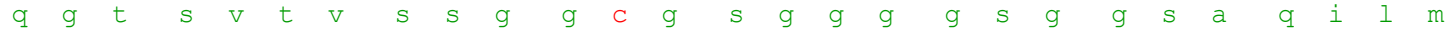

481 acccagtctc ctgcttcctc agttgtatct ctggggcaga gggccaccat ctcatgcagg gccagcaaaa gtgtcagtac

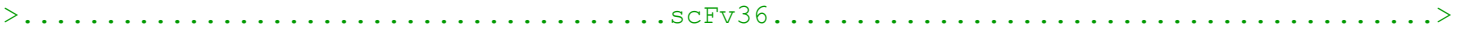

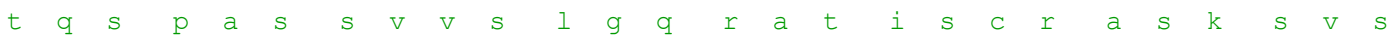

561 atctgcctat agttatatgc actggtacca acagaaacca ggacagccac ccaactcct catctatctt gcatccaacc

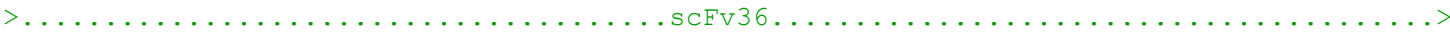

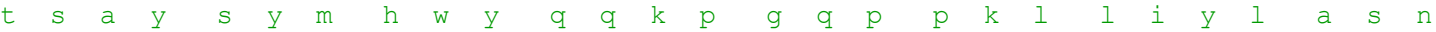


641 tagaatctgg ggtccctccc aggttcagtg gcagtgggtc tgggacagac ttcaccctca acatccaccc tgtggaggag

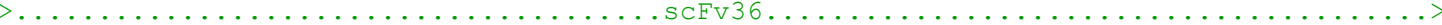

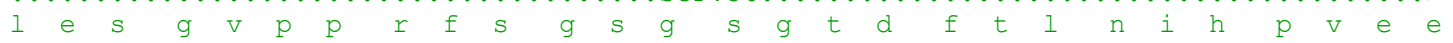

721 gaggatgctg caacctatta ctgtcagcac agtagggagc ttccgtacac gttcggaggg gggaccaagc tggaaataaa

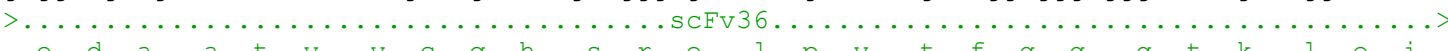

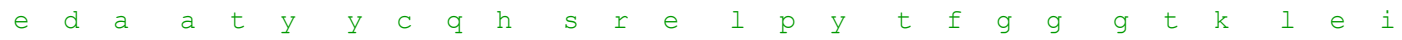

801 acgggcggcc gcagaacaaa aactcatctc agaagaggat ctgaatgggg ccgcacatca ccatcatcac cattaataag His-Taq

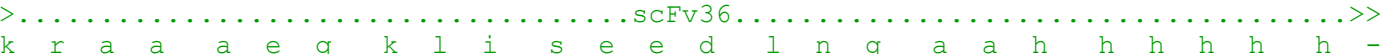

\section{scFv' 36 LCH1}

1 atgaaatacc tattgcctac ggcagccget ggattgttat tactcgcggc ccagccggcc atggcccagg tgcagctgaa

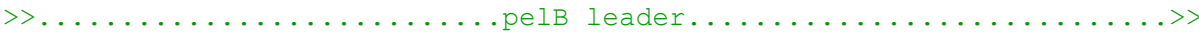

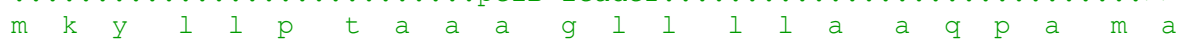
$>>$. ScFv36...>

81 gcagtctgga gctgaactgg tgaaacccgg ggcatcagtg aagctgtcct gcaagacttc tggctacacc ttcactgaaa

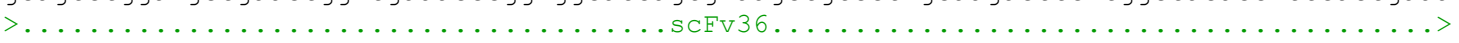

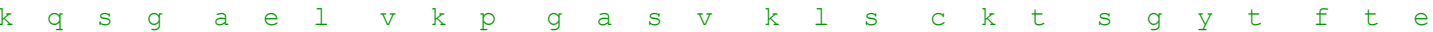

161 atattataca ctgggtaaag cagaggtctg ggcagggtct tgagtggatt gggtggtttc accctggaag tggtagtata

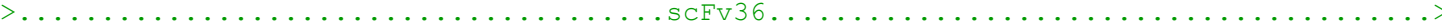

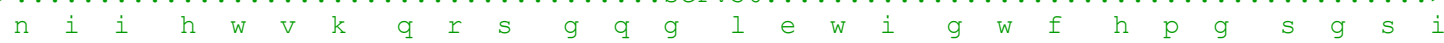

241 aagtacaatg agaaattcaa ggacaaggcc acattgactg cggacaatc ctccagcaca gtctatatgg agcttagtag

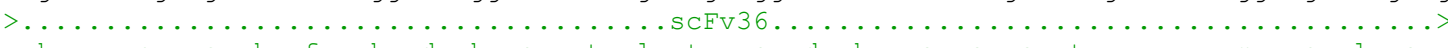

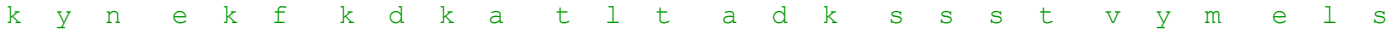

321 attgacatct gaagactctg cggtctattt ctgtgcaaga cacggaggaa ctgggcgagg agctatggac tactggggtc

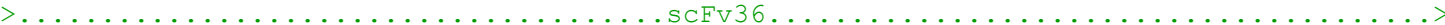

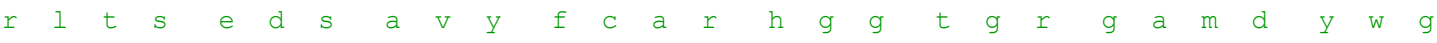

401 aaggaacctc agtcaccgtc tcgagttgcg gaggcggtca tcatcaccat caccatggag gcggtagtgc acaaattctg Cys His-Taq Linker

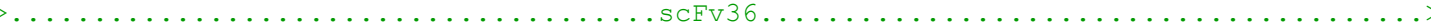

481 atgacccagt ctcctgcttc ctcagttgta tctctggggc agagggccac catctcatgc agggccagca aagtgtcag

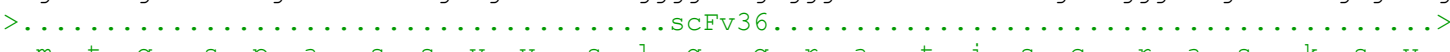

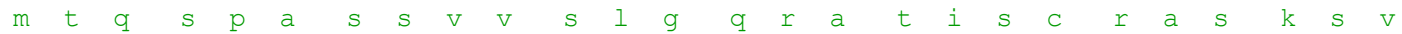

561 tacatctgcc tatagttata tgcactggta ccaacagaaa ccaggacagc cacccaact cctcatctat cttgcatcca

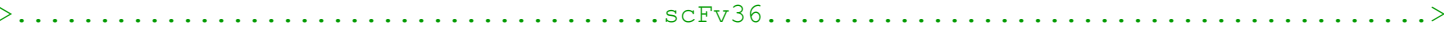

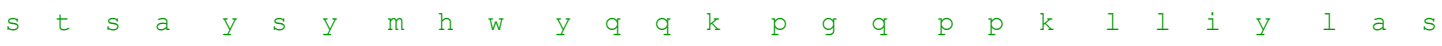

641 acctagaatc tggggtccct cccaggttca gtggcagtgg gtctgggaca gacttcaccc tcaacatcca ccctgtggag

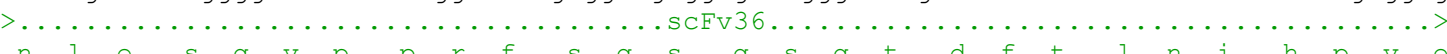

721 gaggaggatg ctgcaaccta ttactgtcag cacagtaggg agcttccgta cacgttcgga ggggggacca agctggaat

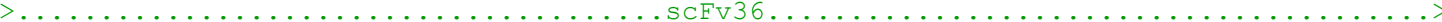

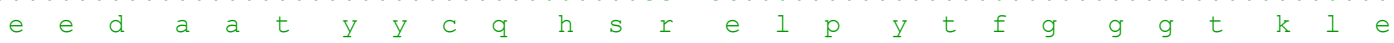

801 aaacggtaa

$>$. SCFV36>>

i $k$ r -

\section{ScFv' 36 LCH3}

1 atgaaatacc tattgcctac ggcagccgct ggattgttat tactcgcggc ccagccggcc atggcccagg tgcagctgaa

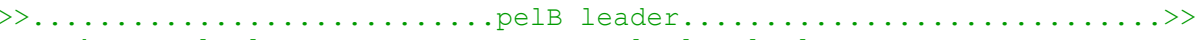

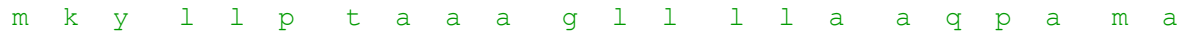
$>>$. scFv36...> q $\quad v \quad q \quad l$ 
81 gcagtctgga gctgaactgg tgaaacccgg ggcatcagtg aagctgtcct gcaagacttc tggctacacc ttcactgaaa

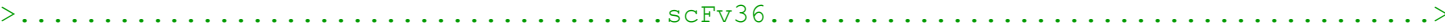

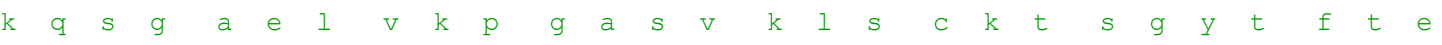

161 atattataca ctgggtaaag cagaggtctg ggcagggtct tgagtggatt gggtggtttc accctggaag tggtagtata

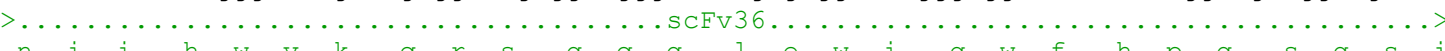

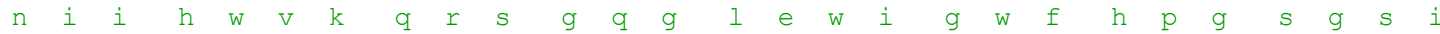

241 aagtacaatg agaaattcaa ggacaaggcc acattgactg cggacaatc ctccagcaca gtctatatgg agcttagtag

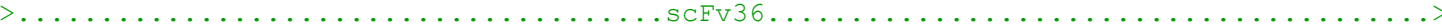

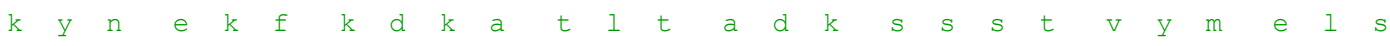

321 attgacatct gaagactctg cggtctattt ctgtgcaaga cacggaggaa ctgggcgagg agctatggac tactggggtc

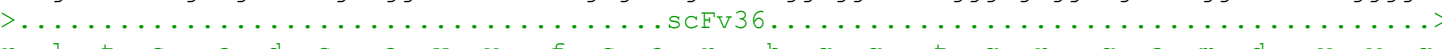

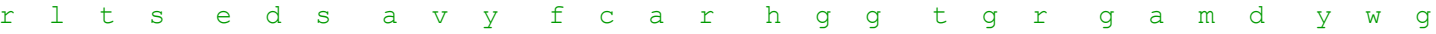

401 aaggaacctc agtcaccgtc tcgagtggtg gatgcggtca tcatcaccat caccatggag gcggtagtgc acaattctg Cys His-Taq Linker

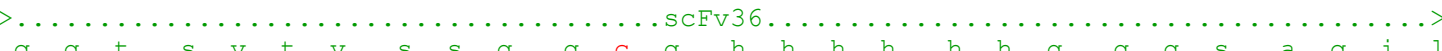

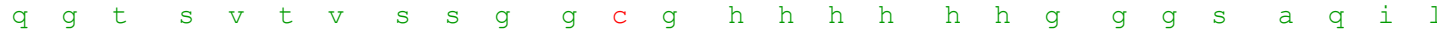

481 atgacccagt ctcctgcttc ctcagttgta tctctgggge agagggccac catctcatgc agggccagca aagtgtcag

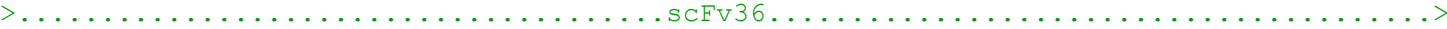

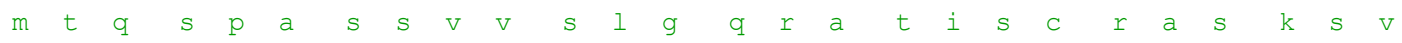

561 tacatctgc tatagttata tgcactggta ccaacagaaa ccaggacagc cacccaact cctcatctat cttgcatcca

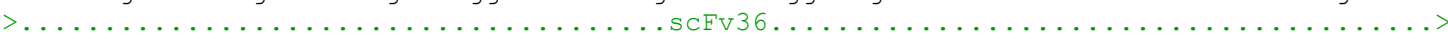

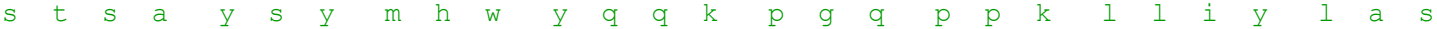

641 acctagaatc tggggtccct cccaggttca gtggcagtgg gtctgggaca gacttcaccc tcaacatcca ccctgtggag

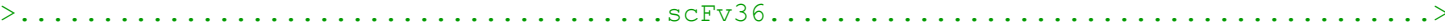

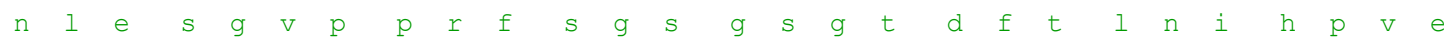

721 gaggaggatg ctgcaaccta ttactgtcag cacagtaggg agcttccgta cacgttcgga ggggggacca agctggaaat

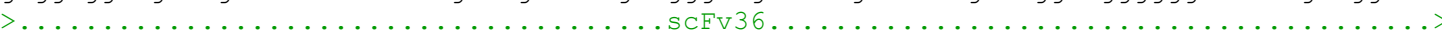

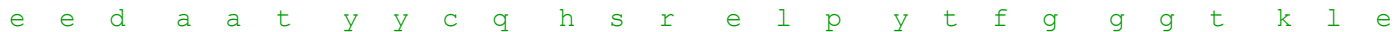

801 aaacggtaa

$>$. SCFV36>>

i $\mathrm{k} r$

\section{Cys-scTNF}

81 tcacacagaa ttcattaaag aggagaaatt aactatgtgc ggatcgcatc accatcacca tcacggatca gcgtcgtctt Cys His-Taq $>\ldots \ldots \ldots \ldots \ldots \ldots \ldots \ldots \ldots \ldots$

161 cttctcgtac cccgtctgac aaaccggttg ctcacgttgt tgcaaacccg caggctgaag gtcaactgca atggctgaac

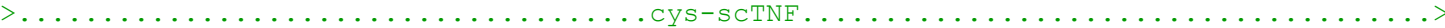

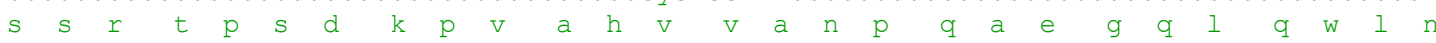

241 cgtcgtgcta acgctctgct ggctaacggt gttgaactgc gtgacaacca gctggttgtt ccgtctgaag gcctgtacct

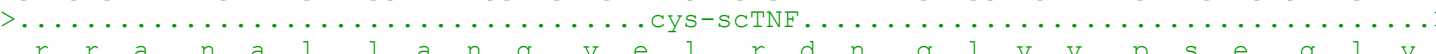

321 gatctactcc caggttctgt tcaaaggcca gggetgcccg tccacccacg ttctgctgac ccacaccatc tctcgtatcg

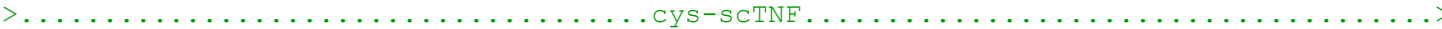

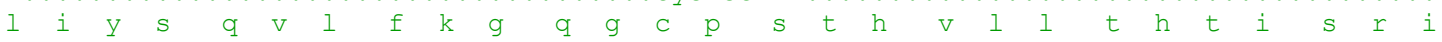

401 ctgtttccta ccagaccaaa gtaaacctgc tgtctgcaat caaatctccg tgccagcgtg aaaccccgga aggtgctgaa

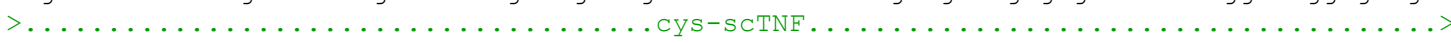

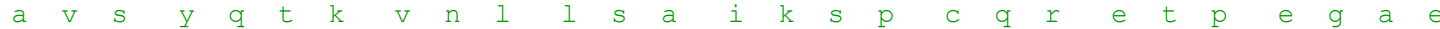

481 gctaaaccgt ggtacgaacc gatctacctg ggtggcgttt ttcaactgga gaaaggtgac cgtctgtctg cagaaattaa

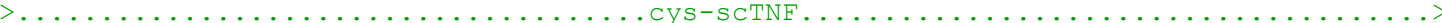

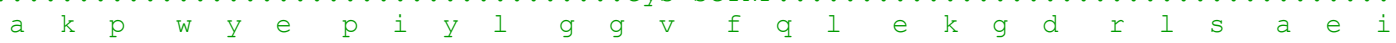

561 ccgtccggac tacctggact tcgcagaatc tggtcaggtt tacttcggta tcatcgctct gggtggcggt tctggtggcg

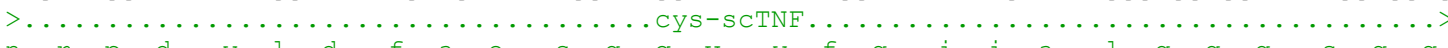

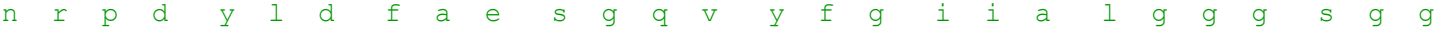


641 gttctggtgg cggttctggt ggcggatcct cttctcgtac cccgtctgac aaaccggttg ctcacgttgt tgcaaacceg

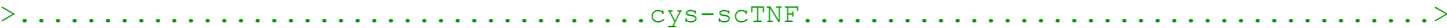

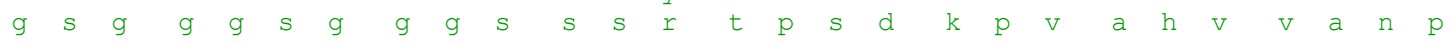

721 caggctgaag gtcaactgca atggctgaac cgtcgtgcta acgctctgct ggctaacggt gttgaactgc gtgacaacca

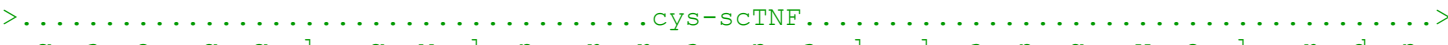

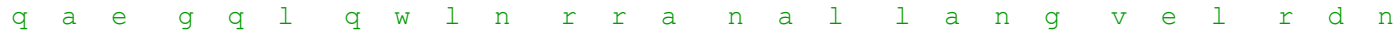

801 gctggttgtt ccgtctgaag gcctgtacct gatctactcc caggttctgt tcaaaggcca gggctgcccg tccacccacg

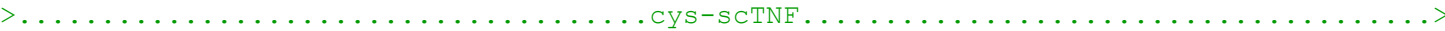

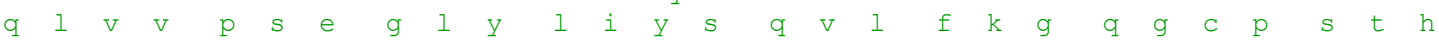

881 ttctgctgac ccacaccatc tctcgtatcg ctgtttccta ccagaccaaa gtaaacctgc tgtctgcaat caaatctccg

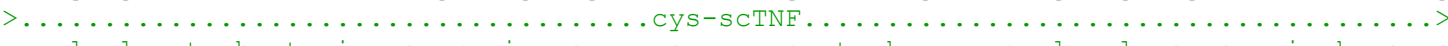

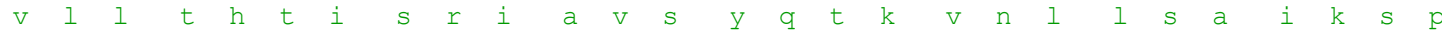

961 tgccagcgtg aaaccccgga aggtgctgaa gctaaaccgt ggtacgaacc gatctacctg ggtggcgttt ttcaactgga

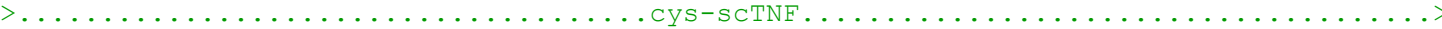

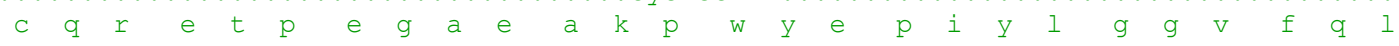

1041 gaaaggtgac cgtctgtctg cagaaattaa ccgtccggac tacctggact tcgcagaatc tggtcaggtt tacttcggta

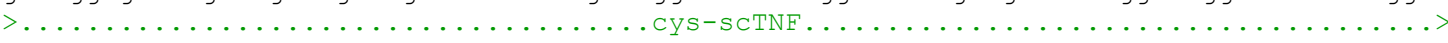

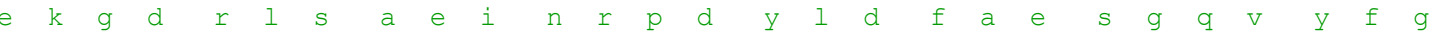

1121 tcatcgctct gggtggcggt tctggtggcg gttctggtgg cggttctggt ggcggatcct cttctcgtac cccgtctgac

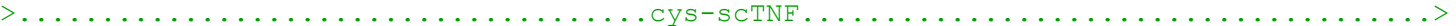

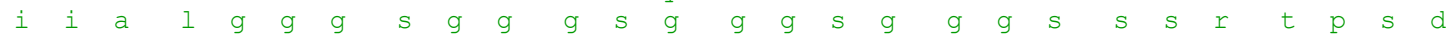

1201 aaaccggttg ctcacgttgt tgcaaacccg caggctgaag gtcaactgca atggctgaac cgtcgtgcta acgetctgct

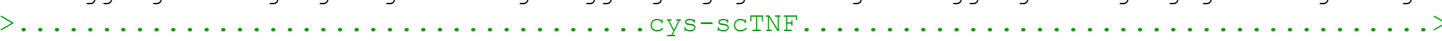

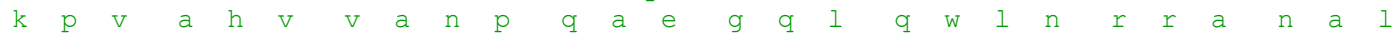

1281 ggctaacggt gttgaactgc gtgacaacca gctggttgtt ccgtctgaag gcctgtacct gatctactcc caggttctgt

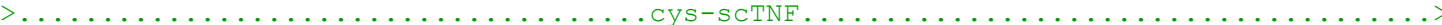

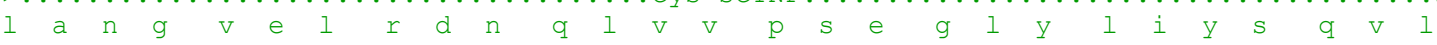

1361 tcaaaggcca gggctgcccg tccacccacg ttctgctgac ccacaccatc tctcgtatcg ctgtttccta ccagaccaaa

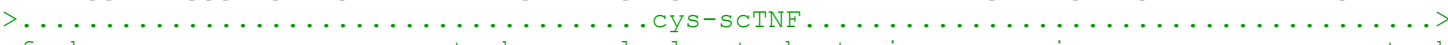

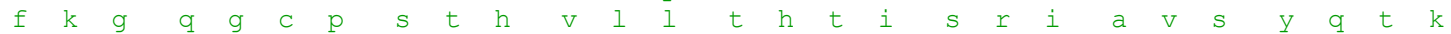

1441 gtaaacctgc tgtctgcaat caaatctccg tgccagcgtg aaaccccgga aggtgctgaa gctaaaccgt ggtacgaacc

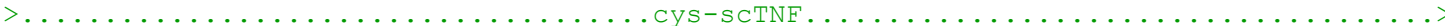

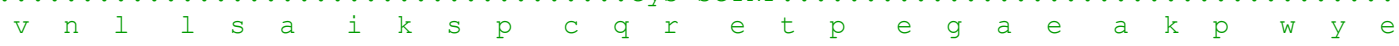

1521 gatctacctg ggtggcgttt ttcaactgga gaaaggtgac cgtctgtctg cagaaattaa ccgtccggac tacctggact

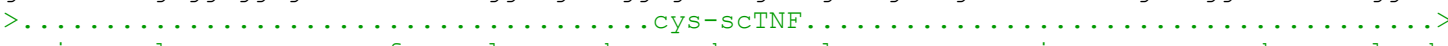

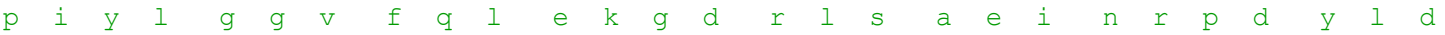

1601 tcgcagaatc tggtcaggtt tactcogta tcatcgctct gtga $\ldots \ldots \ldots \ldots \ldots \ldots$. Cys $-\mathrm{scTNF} \ldots \ldots \ldots \ldots$

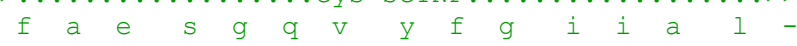




\section{Acknowledgements}

First of all, I wish to thank Roland Kontermann for letting me perfom this interesting research project and for providing me with continuous support through the ups and downs of my work.

Moreover, I want to thank Peter Scheurich and Klaus Pfizenmayer for their help and advice.

I would like to thank Dafne Müller for her valuable technical and theoretical support.

Also l'd like to thank Katharina Landfester, Anna Mysyanovych and Grit Baier for the just in time production of particles.

Thanks a lot to Verena Boschert for investing time to correct my thesis, for answering my questions and not to forget for testing a numerous emergency showers.

Furthermore, I want to thank Margarete Witowski for her help with microscopy and Sabine Münkel for her kind support with HPLC.

I thank all former and present colleagues from the Kontermann and the Scheurich group for stimulating discussions, knowledge exchange and for the very enjoyable working atmosphere at the institute.

I wish to thank all IZI-members for their nice support and countless coffee- and cake-breaks.

Finally, I wish to express my gratitude to my family for all their support during my studies. I thank Regina for several motivation telephone-calls and the $2^{\text {nd }}$ hand shopping tours. Thanks a lot to Petra for always having an open ear during my $\mathrm{PhD}$ thesis. Last but not least, I wish to thank Wolfgang for his love and attendance on my way towards the $\mathrm{PhD}$. 


\title{
Curriculum Vitae
}

\author{
Persönliche Daten: \\ Name: \\ Sylvia Messerschmidt geb. Adebahr \\ Geburtsdatum: 11. August 1980 \\ Geburtsort: $\quad$ Neustadt an der Weinstraße
}

Schulausbildung:

87-91

Grund- und Hauptschule Hirschlanden - Schöckingen

$91-00$

Mädchengymnasium St. Agnes Stuttgart, Abschluß: Abitur

\begin{abstract}
Akademische Ausbildung:
10/00-03/06 Studium der Technisch orientieren (t.o.) Biologie an der Universität Stuttgart Hauptfach: Immunologie

Nebenfächer: Tierphysiologie, Mirkobiologie

Wahlpflichtfach: Biochemie

03/06

Studienabschluss zur Diplom-Biologin, technisch orientiert (t.o.)

04/06-03/09

Doktorarbeit am Institut für Zellbiologie und Immunologie

Arbeitsgruppe von Prof. Dr. Roland Kontermann
\end{abstract}

\section{Praktische Tätigkeiten:}

10/04-02/05

Studienarbeit am Biologischen Institut Abteilung Botanik

Titel: Die Bedeutung der Fettsäurenzusammensetzung für die Frosttoleranz bei Arabidopsis thaliana.

10-11/04 Studentische Hilfskraft am Biologischen Institut Abteilung Botanik

02-03/05 Industriepraktikum bei JOHANNES LIEDER GmbH \& Co.KG

05/05-03/06 Diplomarbeit am Institut für Zellbiologie und Immunologie

Titel: Die Rolle der Stiel- und transmembranären Region der TNF-Rezeptoren an der differenziellen Antwortfähigkeit für lösliches versus membrangeb. TNF.

04/06-03/09 Doktorarbeit am Institut für Zellbiologie und Immunologie

Titel: Targeted lipid-coated nanoparticles: delivery of tumor necrosis factor functionalized particles to tumor cells.

Auszeichnungen und Publikationen:

03/09

Posterpreis, Controlled Release Society German Chapter Annual Meeting, Halle (Saale).

Messerschmidt SKE, Musyanovych A, Altvater M, Scheurich P, Pfizenmaier K, Landfester K \& Kontermann RE. (2009) Targeted lipid-coated nanoparticles: Delivery of tumor necrosis factorfunctionalized particles to tumor cells. Journal of Controlled Release, in press.

Messerschmidt SKE, Beuttler J, Rothdiener M \& Kontermann RE. (2009) Recombinant antibody molecules in nanobiotechnology: immunoliposomes. In: Springer protocols antobody engineering. Kontermann RE \& Dübel S (Ed.) Springer Verlag Heidelberg.

Messerschmidt SKE, Kolbe A, Müller D, Knoll M, Pleiss J \& Kontermann RE. (2008) Novel singlechain Fv' formats for the generation of immunoliposomes by site-directed coupling. Bioconjug. Chem. 19, 362-369.

Rothdiener M, Beuttler J, Messerschmidt SKE \& Kontermann RE. (2009) Antibody targeting of nanoparticles to tumor specific receptors: immunoliposomes. In: Cancer nanotechnology in Methods in molecular biology series. Grobmyer S \& Moudgil B (Ed.) Humana Pres Inc.

Korntal, im April 2009 